\title{
Equilibrium between radiation and matter for classical relativistic multiperiodic systems. Derivation of Maxwell-Boltzmann distribution from Rayleigh-Jeans spectrum
}

\author{
R. Blanco, L. Pesquera, and E. Santos \\ Departamento de Física Teórica, Universidad de Santander, Santander, Spain
}

(Received 24 June 1982)

\begin{abstract}
The motion of a charged pointlike relativistic particle under the action of a given force field plus a random electromagnetic radiation is studied. It is assumed that the given force field alone should produce a multiply periodic motion, which is perturbed by the action of both the random radiation and the reaction damping. The random radiation is represented by a stochastic process and an equation is obtained for the equilibrium probability density of the particle in phase space. In the particular case of a random radiation with Rayleigh-Jeans spectrum, it is shown that the stationary solution, corresponding to radiation-matter equilibrium, is given by the Maxwell-Boltzmann distribution.
\end{abstract}

\section{INTRODUCTION}

The derivation of the classical blackbody spectrum (Rayleigh-Jeans law) was made between 1900 and 1905 with the works of Rayleigh, Einstein, and Jeans. In the years that followed, Einstein himself and others studied different models to obtain the classical spectral law, a subject extremely important because the disagreement of that law with empirical evidence was historically the origin of the quantum revolution. ${ }^{1}$ The subject was considered to be definitely settled in 1924 with the work of Van Vleck. ${ }^{2}$ However, strangely enough, all papers dealt with nonrelativistic theory. Recently, Boyer ${ }^{3}$ has claimed that a classical relativistic treatment leads to a contradiction between the Rayleigh-Jeans law for thermal radiation and the Maxwell-Boltzmann distribution. The purpose of this paper is to report a consistent relativistic derivation which proves that the Rayleigh-Jeans law does indeed lead to the Maxwell-Boltzmann distribution. It is interesting to note that a similar situation has arisen with the quantal law. In fact, Boyer ${ }^{4}$ also claimed that there was a contradiction between quantum theory, relativity, and statistical mechanics, which has been shown to be not true. ${ }^{5}$

The interest in making a classical relativistic derivation of the blackbody spectrum is the following. In the first place, it is different to say that classical laws do not agree with experiments (e.g., they lead to the Rayleigh-Jeans law instead of the correct Planck law), which is well known, than to say that the classical postulates are selfcontradictory, which should deny the possibility of a fully relativistic classical statistical mechanics. In the second place, some doubts have arisen with respect to whether the Planck law, instead of the Rayleigh-Jeans law, could not be derived from classical postulates provided that one includes a zero-point radiation with an $|\omega|^{3}$ spectrum (i.e., the same spectrum of the fluctuation field of the vacuum in quantum electrodynamics). This might lead to a stochastic alternative to quantum theory ${ }^{6}$ (usually called stochastic or random electrodynamics $^{7}$ ).

In this paper we show that the stationary state of a point charge without structure in the presence of a random electromagnetic radiation with spectrum constant $\times \omega^{2}$, and other forces, has a phasespace distribution function given by the MaxwellBoltzmann law,

$$
W_{0}(q, p)=\text { const } \times \exp \left(-\mathscr{E} / \mathscr{C}_{0}\right)
$$

where $\mathscr{E}$ stands for the deterministic relativistic energy of the charge. With regard to the other forces present in the problem, we only consider multiply periodic systems. In a second part of this work, to be published later, we shall prove that, with these conditions, the Rayleigh-Jeans and MaxwellBoltzmann laws, the charge is in perfect energetic equilibrium with the random electromagnetic radiation at each frequency.

The plan of this paper is as follows. In Sec. II, 
we give the mathematical formulation of the problem. We present the model of electromagnetic background radiation and several properties of multiply periodic motions, which will be needed later. In Sec. III, we review methods for the solution of stochastic differential equations which are neither linear nor Markovian.

These are used in Sec. IV in order to obtain a Fokker-Planck equation. The coefficients of that equation are obtained in Sec. V and, finally, in Sec. VI we calculate the solution of the Fokker-Planck equation. Several details of the calculations are given in Appendices A, B, and C, while in Appendix D we analyze the special case of a central potential.

\section{FORMULATION OF THE PROBLEM}

\section{A. Equations of motion}

We wish to study the motion of a charged point particle (i.e., structureless) interacting with an electromagnetic radiation field. We assume that there are other forces, besides the ones of the radiation field, acting upon the particle. We shall consider conservative forces satisfying the following conditions:

(i) The potential increases at infinity quickly enough.

(ii) Under the action of this potential alone, the motion should be multiply periodic.

With respect to the first condition, it is true that the usual potentials do not fulfil it, but, when we consider equilibrium with radiation, we must take the system as being enclosed in some "box", which is equivalent to putting an infinite potential outside the box. On the other hand, the second condition will allow us to analyze the motion in terms of frequencies, which will be needed in the second part of this work. In particular, every central force field is included under the second condition. Finally, condition (i) guarantees that all states of the system are bounded, i.e., for any energy $|\overrightarrow{\mathbf{r}}(t ; \mathscr{E})|$ is bounded by a finite value.

The differential equation of the motion of the charged point particle is the Lorentz-Dirac equation $^{8}$ (here, the signature is +2 )

$$
m \alpha^{\mu}=F_{\mathrm{det}}^{\mu}+F_{\mathrm{st}}^{\mu}+\frac{2 e^{2}}{3 c^{3}}\left(\frac{d \alpha^{\mu}}{d \tau}-\frac{1}{c^{2}} \alpha_{v} \alpha^{v} u^{\mu}\right)
$$

where $\tau$ is the proper time, $u^{\mu}$ and $\alpha^{\mu}$, are the four-velocity and the four-acceleration, respectively, and $F^{\mu}$ is the force four-vector. The relations with the usual three-vectors are

$$
\begin{aligned}
u^{\mu} & =(\gamma c, \gamma \overrightarrow{\mathrm{v}}), \\
\alpha^{\mu} & =\left(\gamma^{4} \frac{\overrightarrow{\mathrm{v}} \cdot \overrightarrow{\mathrm{a}}}{c}, \gamma^{2} \overrightarrow{\mathrm{a}}+\gamma^{4} \frac{\overrightarrow{\mathrm{v}} \cdot \overrightarrow{\mathrm{a}}}{c^{2}} \overrightarrow{\mathrm{v}}\right), \\
F^{\mu} & =\left[\gamma \frac{1}{c} \overrightarrow{\mathrm{v}} \cdot \overrightarrow{\mathrm{F}}, \gamma \overrightarrow{\mathrm{F}}\right] .
\end{aligned}
$$

The first term on the right-hand side of (2.1) represents the force due to the potential $V(\overrightarrow{\mathrm{r}})$ which we shall call deterministic force. The second term is the force of the radiation field on the particle, which could be written as a Lorentz force

$$
\overrightarrow{\mathrm{F}}^{\mathrm{st}}=e\left(\overrightarrow{\mathrm{E}}(\overrightarrow{\mathrm{r}}, t)+\frac{\overrightarrow{\mathrm{v}} \times \overrightarrow{\mathrm{B}}(\overrightarrow{\mathrm{r}}, t)}{c}\right) .
$$

The fields $\overrightarrow{\mathrm{E}}(\overrightarrow{\mathrm{r}}, t)$ and $\overrightarrow{\mathrm{B}}(\overrightarrow{\mathrm{r}}, t)$ provide the thermal bath in which our system is immersed, and they will be considered random fields whose statistical properties will be stated below.

Finally, the last term is the damping due to the radiation reaction. The Lorentz-Dirac expression used in Equation (2.1) is the standard one for a point particle.

If we use the time $t$ of a fixed frame instead of the proper time $\tau$, Eq. (2.1) can be written in terms of the (mechanical) linear momentum

$$
\overrightarrow{\mathrm{p}}=m \gamma \overrightarrow{\mathrm{v}}=m\left(1-v^{2} / c^{2}\right)^{-1 / 2} \overrightarrow{\mathrm{v}}
$$

as

$$
\begin{aligned}
\dot{\overrightarrow{\mathrm{r}}}= & \frac{\overrightarrow{\mathrm{p}} c}{\left(p^{2}+m^{2} c^{2}\right)^{1 / 2}}(=\overrightarrow{\mathrm{v}}), \\
\dot{\overrightarrow{\mathrm{p}}}= & \overrightarrow{\mathrm{F}}^{\mathrm{det}}+\frac{2 e^{2}}{3 c^{3}}\left[\dot{\vec{\alpha}}-\frac{1}{c^{2}} \alpha_{\nu} \alpha^{v} \overrightarrow{\mathrm{v}}\right) \\
& +e\left(\overrightarrow{\mathrm{E}}(\overrightarrow{\mathrm{r}}, t)+\frac{\overrightarrow{\mathrm{v}} \times \overrightarrow{\mathrm{B}}(\overrightarrow{\mathrm{r}}, t)}{c}\right),
\end{aligned}
$$

where an overdot means $d / d t$ and $\vec{\alpha}$ is the spatial part of $\alpha^{\mu}$.

Although we consider here deterministic forces which derive from a velocity-independent potential, it is straightforward to generalize to potentials which depend linearly on the velocities, provided that we use the canonical momentum of the deter- 
ministic motion and that the deterministic equations fulfil the condition of being multiperiodic.

\section{B. Stochastic properties of the radiation field}

Any radiation field in a space without charges can be written as a sum of plane waves [the field of the charge under consideration is taken into account in the last term of Eq. (2.1), not in the second one]. We assume that the amplitudes of the plane waves are statistically independent of each other. Then, there are two primary models for these amplitudes. Either we assume amplitudes with a fixed modulus and phases at random (i.e., with the same probability for each $\varphi$ between 0 and $2 \pi),{ }^{9}$ or we assume that the moduli are Gaussian random variables with random phases. ${ }^{10}$

A Gaussian random variable with zero mean is fully characterized by its standard derivation, therefore a single number gives all information about the probability distribution of the amplitudes of a plane wave. Actually, both models, although apparently different, lead to the same correlations for the random fields, so that both models are equivalent. We shall use the first model, following Boyer.

The random field will be written

$$
\begin{aligned}
\overrightarrow{\mathrm{E}}(\overrightarrow{\mathrm{r}}, t)=\sum_{\lambda=1}^{2} \int & d^{3} k \vec{\epsilon}(\overrightarrow{\mathrm{k}}, \lambda) \mathscr{Y}(\overrightarrow{\mathrm{k}}) \\
& \times \cos [\overrightarrow{\mathrm{k}} \cdot \overrightarrow{\mathrm{r}}-\omega t+\theta(\overrightarrow{\mathrm{k}}, \lambda)], \\
\overrightarrow{\mathbf{B}}(\overrightarrow{\mathrm{r}}, t)=\sum_{\lambda=1}^{2} \int & d^{3} k \frac{\overrightarrow{\mathrm{k}} \times \vec{\epsilon}(\overrightarrow{\mathrm{k}}, \lambda)}{k} \mathscr{Y}(\overrightarrow{\mathrm{k}}) \\
& \times \cos [\overrightarrow{\mathrm{k}} \cdot \overrightarrow{\mathrm{r}}-\omega t+\theta(\overrightarrow{\mathrm{k}}, \lambda)],
\end{aligned}
$$

where $\theta$ is a random variable satisfying

$$
\begin{aligned}
d P_{\theta}(\theta)=\frac{1}{2 \pi} d \theta & \\
\left\langle\cos \theta(\overrightarrow{\mathrm{k}}, \lambda) \cos \theta\left(\overrightarrow{\mathrm{k}}^{\prime}, \lambda^{\prime}\right)\right\rangle & =\left\langle\sin \theta(\overrightarrow{\mathrm{k}}, \lambda) \sin \theta\left(\overrightarrow{\mathrm{k}}^{\prime}, \lambda^{\prime}\right)\right\rangle \\
& =\frac{1}{2} \delta_{\lambda \lambda^{\prime}} \delta^{3}\left(\overrightarrow{\mathrm{k}}-\overrightarrow{\mathrm{k}}^{\prime}\right) \\
\left\langle\cos \theta(\overrightarrow{\mathrm{k}}, \lambda) \sin \theta\left(\overrightarrow{\mathrm{k}}^{\prime}, \lambda^{\prime}\right)\right\rangle & =0 .
\end{aligned}
$$

Polarization vectors are such that

$$
\begin{aligned}
& \overrightarrow{\mathrm{k}} \cdot \vec{\epsilon}(\overrightarrow{\mathrm{k}}, \lambda)=0, \\
& \vec{\epsilon}(\overrightarrow{\mathrm{k}}, \lambda) \cdot \vec{\epsilon}\left(\overrightarrow{\mathrm{k}}, \lambda^{\prime}\right)=\delta_{\lambda \lambda^{\prime}}, \\
& \sum_{\lambda=1}^{2} \epsilon_{i}(\overrightarrow{\mathrm{k}}, \lambda) \epsilon_{j}(\overrightarrow{\mathrm{k}}, \lambda)=\delta_{i j}-\frac{k_{i} k_{j}}{k^{2}} .
\end{aligned}
$$

With all this, the following correlations are obtained:

$$
\begin{aligned}
\left\langle E_{i}\left(\overrightarrow{\mathrm{r}}_{1}, t_{1}\right) E_{j}\left(\overrightarrow{\mathrm{r}}_{2}, t_{2}\right)\right\rangle & =\left\langle B_{i}\left(\overrightarrow{\mathrm{r}}_{1}, t_{1}\right) B_{j}\left(\overrightarrow{\mathrm{r}}_{2}, t_{2}\right)\right\rangle \\
& =\int d^{3} k\left[\delta_{i j}-\frac{k_{i} k_{j}}{k^{2}}\right] \frac{\mathscr{Y}^{2}(\overrightarrow{\mathrm{k}})}{2} \cos \left[\overrightarrow{\mathrm{k}} \cdot\left(\overrightarrow{\mathrm{r}}_{1}-\overrightarrow{\mathrm{r}}_{2}\right)-\omega\left(t_{1}-t_{2}\right)\right], \\
\left\langle E_{i}\left(\overrightarrow{\mathrm{r}}_{1}, t_{1}\right) B_{j}\left(\overrightarrow{\mathrm{r}}_{2}, t_{2}\right)\right\rangle & =\int d^{3} k \epsilon_{i j l} \frac{k_{l}}{k} \frac{\mathscr{Y}^{2}(\overrightarrow{\mathrm{k}})}{2} \cos \left[\overrightarrow{\mathrm{k}} \cdot\left(\overrightarrow{\mathrm{r}}_{1}-\overrightarrow{\mathrm{r}}_{2}\right)-\omega\left(t_{1}-t_{2}\right)\right] .
\end{aligned}
$$

By homogeneity and isotropy the quantity $\mathscr{Y}(\overrightarrow{\mathrm{k}})$ depends only on the frequency. This dependence can be found by comparison between the energy density of the field per unit frequency interval and the spectrum of the field defined as the Fourier transform of the correlation.

(a) The energy density of an electromagnetic field is given by

$$
U=\frac{1}{8 \pi}\left\langle E^{2}+B^{2}\right\rangle
$$

We wish to calculate the energy density corresponding to all the plane waves which have frequencies between $\omega$ and $\omega+\Delta \omega$. The fields corresponding to those waves are

$$
\begin{aligned}
& \overrightarrow{\mathrm{E}}_{\omega}=\int_{\omega}^{\omega+\Delta \omega} d \omega^{\prime} \frac{\mathscr{Y}^{2}\left(\omega^{\prime}\right) \omega^{\prime 2}}{c^{3}} \int d \Omega_{\overrightarrow{\mathrm{k}}} \vec{\epsilon}(\overrightarrow{\mathrm{k}}, \lambda) \cos \left[\overrightarrow{\mathrm{k}} \cdot \overrightarrow{\mathrm{r}}-\omega^{\prime} t+\theta(\overrightarrow{\mathrm{k}}, \lambda)\right], \\
& \overrightarrow{\mathrm{B}}_{\omega}=\int_{\omega}^{\omega+\Delta \omega} d \omega^{\prime} \frac{\mathscr{Y}^{2}\left(\omega^{\prime}\right) \omega^{\prime 2}}{c^{3}} \int d \Omega_{\overrightarrow{\mathrm{k}}} \frac{\overrightarrow{\mathrm{k}} \times \vec{\epsilon}(\overrightarrow{\mathrm{k}}, \lambda)}{k} \cos \left[\overrightarrow{\mathrm{k}} \cdot \overrightarrow{\mathrm{r}}-\omega^{\prime} t+\theta(\overrightarrow{\mathrm{k}}, \lambda)\right],
\end{aligned}
$$


where $\omega^{\prime}=c|\overrightarrow{\mathrm{k}}|$. Using Eqs. (2.3) and (2.4), we obtain

$$
\begin{aligned}
U_{(\omega, \omega+\Delta \omega)} & =\frac{1}{8 \pi}\left\langle E_{\omega}{ }^{2}+B_{\omega}{ }^{2}\right\rangle \\
& =\int_{\omega}^{\omega+\Delta \omega} d \omega^{\prime} \frac{\mathscr{Y}^{2}\left(\omega^{\prime}\right) \omega^{\prime 2}}{c^{3}}
\end{aligned}
$$

Finally, taking $\Delta \omega \rightarrow 0$, it turns out that

$$
U(\omega)=\lim _{\Delta \omega \rightarrow 0} \frac{U_{(\omega, \omega+\Delta \omega)}}{\Delta \omega}=\frac{\mathscr{Y}^{2}(\omega) \omega^{2}}{c^{3}}
$$

(b) The spectrum of the radiation is defined by

$$
S_{i}(\omega)=\int_{-\infty}^{+\infty}\left\langle E_{i}(\overrightarrow{\mathrm{r}}, t) E_{i}(\overrightarrow{\mathrm{r}}, t-\tau)\right\rangle e^{i \omega \tau} d \tau
$$

Using Eq. (2.5) and performing the integration, we obtain

$$
S_{i}(\omega)=\frac{4 \pi^{2}}{3} \frac{\omega^{2} \mathscr{Y}^{2}(\omega)}{c^{3}}
$$

independently of the coordinate, which is a consequence of the isotropy. Then, we have

$$
S(\omega)=S_{i}(\omega)=\frac{4 \pi^{2}}{3} U(\omega),
$$

which is the desired relation between the energy density and the spectrum.

\section{Properties of multiperiodic motions}

Here we summarize the most relevant properties in order to fix the notation we use.

When the Hamilton-Jacobi equation is separable and the paths in each plane $\left(q_{i}, p_{i}\right)$ are closed or periodic, it is possible to define angle and action variables in the following way.

Action variables are defined by

$$
J_{i}=\frac{1}{2 \pi} \oint p_{i} d q_{i}
$$

where the integral goes over a circle or period of the path. If the Hamilton characteristic function ${ }^{11}$ is written as

$$
W=\sum_{i} W_{i}\left(q_{i}, \alpha_{1} \cdots \alpha_{n}\right),
$$

then, by the transformation equations, we have

$$
p_{i}=\frac{\partial W}{\partial q_{i}}=W_{i}^{\prime}\left(q_{i}, \alpha_{1} \cdots \alpha_{n}\right),
$$

whereby $J_{i}$ depends only on $\alpha$ and it is, therefore, a constant of the motion. Choosing now $J$ as the new momenta, we define

$$
w_{i}=\frac{\partial W}{\partial J_{i}} \text {. }
$$

It is possible to show that if one of the $q_{i}$ moves through a period, the corresponding $w_{j}$ changes by $2 \pi$ while the remaining $w$ do not change. Also

$$
\dot{w}_{i}=\frac{\partial \mathscr{E}}{\partial J_{i}} \text { and } \mathscr{E}=\mathscr{E}\left(J_{1}, \ldots, J_{n}\right)
$$

so that $\dot{w}_{i}$ is a constant whence

$$
w_{i}(t)=w_{i}^{0}+\omega_{i}(\overrightarrow{\mathrm{J}}) t \text {. }
$$

Then, each $q_{i}$ is a periodic function of $w_{i}$ and therefore of $t$, i.e.,

$$
\begin{aligned}
q_{i} & =\sum_{n} q_{i, n} e^{i n w_{i}} \\
& =\sum_{n} q_{i, n} e^{i n w_{i}^{0}} e^{i n \omega_{i} t} .
\end{aligned}
$$

As $p_{i}=W_{1}^{\prime}\left(q_{i}, J_{1}, \ldots, J_{n}\right), p_{i}$ is also a periodic function of $w_{i}$ and, finally, every function defined in phase space will be a periodic function of the $\vec{w}$,

$$
f(\overrightarrow{\mathrm{r}}, \overrightarrow{\mathrm{p}})=\sum_{\overrightarrow{\mathrm{n}}} f_{\overrightarrow{\mathrm{n}}} e^{i \overrightarrow{\mathrm{w}}(t) \cdot \overrightarrow{\mathrm{n}}},
$$

where $\overrightarrow{\mathrm{n}} \cdot \overrightarrow{\mathrm{w}}$ means $\sum n_{i} w_{i}$ and $n_{i}$ is an integer.

Some care is needed if there is degeneration, i.e., when a relation exists of the type $\sum_{i} n_{i} \omega_{i}=0$ with not all $n_{i}$ zero. In this case it can be shown ${ }^{11}$ that a linear canonical transformation exists such that the new coordinates and momenta have the same properties as before, but for each of the abovementioned relations, it is possible to replace one of the $w_{i}$ by one constant. Then, we can assume that only $M$ of the frequencies are incommensurable, i.e.,

$$
\sum_{i=1}^{M} \omega_{i} n_{i}=0 \Leftrightarrow n_{i}=0, i \leq M
$$

and

$$
k>M \Longrightarrow \omega_{k}=0 .
$$

Then, since we have

$$
\dot{w}_{k}=\omega_{k}=0=\frac{\partial \mathscr{E}}{\partial J_{k}}, k>M
$$

it follows that $\mathscr{E}$ is only a function of the $M$ first $J_{i}$.

We shall use the following notation: $\overrightarrow{\mathrm{w}}$ and $\vec{J}$ 
represent the first $M$ coordinates and momenta

$$
\overrightarrow{\mathbf{w}} \equiv\left(w_{1}, \ldots, w_{M}\right), \quad \overrightarrow{\mathbf{J}} \equiv\left(J_{1}, \ldots, J_{M}\right)
$$

and $\overrightarrow{\mathbf{w}}^{\prime}, \overrightarrow{\mathbf{J}}^{\prime}$, the rest, i.e.,

$$
\overrightarrow{\mathbf{w}}^{\prime} \equiv\left(w_{M+1}, \ldots, w_{n}\right), \quad \overrightarrow{\mathbf{J}}^{\prime} \equiv\left(J_{M+1}, \ldots, J_{n}\right) .
$$

Now, $\overrightarrow{\mathrm{w}} \cdot \overrightarrow{\mathrm{n}}$ means $\sum_{i=1}^{M} w_{i} n_{i}$. Note that $\overrightarrow{\mathrm{w}}^{\prime}, \overrightarrow{\mathrm{J}}$, and $\overrightarrow{\mathbf{J}}^{\prime}$ are constant while

$$
\overrightarrow{\mathbf{w}}=\overrightarrow{\mathbf{w}}^{0}+\vec{\omega}_{0} t, \quad \vec{\omega}_{0} \equiv\left(\omega_{1}, \ldots, \omega_{M}\right) .
$$

Similarly, Eq. (2.8) will be written

$$
\begin{aligned}
f(\overrightarrow{\mathrm{r}}, \overrightarrow{\mathrm{p}}) & =\sum_{n_{1}, \ldots, n_{n}} f_{n_{1}, \ldots, n_{n}} \exp \left(i \sum_{i=1}^{n} w_{i} n_{i}\right) \\
& =\sum_{\overrightarrow{\mathrm{n}}, \overrightarrow{\mathrm{n}}^{\prime}} f_{\overrightarrow{\mathrm{n}}, \overrightarrow{\mathrm{n}}^{\prime}} e^{i \overrightarrow{\mathrm{w}} \cdot \overrightarrow{\mathrm{n}}} e^{i \overrightarrow{\mathrm{w}}^{\prime} \cdot \overrightarrow{\mathrm{n}}^{\prime}} \\
& =\sum_{\overrightarrow{\mathrm{n}}} \bar{f}_{\overrightarrow{\mathrm{n}}} e^{i \overrightarrow{\mathrm{w}} \cdot \overrightarrow{\mathrm{n}}}
\end{aligned}
$$

with

$$
\bar{f}_{\overrightarrow{\mathrm{n}}}=\sum_{\overrightarrow{\mathrm{n}}{ }^{\prime}} f_{\overrightarrow{\mathrm{n}}, \overrightarrow{\mathrm{n}}}, e^{i \overrightarrow{\mathrm{w}}^{\prime} \cdot \overrightarrow{\mathrm{n}}^{\prime}}
$$

because $\overrightarrow{\mathrm{w}}^{\prime}(t)=\overrightarrow{\mathrm{w}}_{0}^{\prime}$ does not depend on $t$.

An important property for the future calculations is the following. For any phase-space function $f$ (or, equivalently, any function depending on positions and velocities), we have

$$
\begin{aligned}
& \frac{1}{(2 \pi)^{M}} \int d \overrightarrow{\mathrm{w}} f(\overrightarrow{\mathrm{r}}, \overrightarrow{\mathrm{p}}) \\
&=\lim _{T \rightarrow \infty} \frac{1}{T} \int_{0}^{T} d t f(\overrightarrow{\mathrm{r}}(t), \overrightarrow{\mathrm{p}}(t)),
\end{aligned}
$$

where

$$
\int d \overrightarrow{\mathrm{w}}=\prod_{i=1}^{M} \int_{0}^{2 \pi} d w_{i}
$$

The proof is trivial starting with $\left(2.8^{\prime}\right)$. This expression will be written as

$$
T[f]=\int d \overrightarrow{\mathbf{w}} f(\overrightarrow{\mathbf{r}}, \overrightarrow{\mathrm{p}}) .
$$

Finally, a general property which is valid for any canonical transformation is ${ }^{12}$

$$
\frac{\partial q_{i}}{\partial Q_{j}}=\frac{\partial P_{j}}{\partial p_{i}}, \frac{\partial q_{i}}{\partial P_{j}}=-\frac{\partial Q_{j}}{\partial p_{i}}, \frac{\partial p_{i}}{\partial Q_{j}}=-\frac{\partial P_{j}}{\partial q_{i}},
$$

which, in our case, gives the relations

$$
\begin{aligned}
& \frac{\partial x_{i}}{\partial w_{j}}=\frac{\partial J_{j}}{\partial p_{i}}, \frac{\partial x_{i}}{\partial J_{j}}=-\frac{\partial w_{j}}{\partial p_{i}} \\
& \frac{\partial w_{i}}{\partial x_{j}}=\frac{\partial p_{j}}{\partial J_{i}}, \frac{\partial p_{i}}{\partial w_{j}}=-\frac{\partial J_{j}}{\partial x_{i}}
\end{aligned}
$$

\section{METHODS OF RESOLUTION OF NON-MARKOVIAN NONLINEAR STOCHASTIC DIFFERENTIAL EQUATIONS}

As we have seen above, a relativistic charged system in a radiation field is described by a differential equation with a stochastic term, which corresponds to the electromagnetic field. Usually, we say that a solution of this stochastic differential equation is found when we obtain an equation for the probability density of the phase-space variables of the system. In most of the physical problems it is enough to know the stationary probability density, and this will be indeed the case in our problem because we are interested in the equilibrium state of a relativistic system with a radiation field.

When the spectral density of the stochastic field is a constant (white-noise), standard methods may be used to obtain an equation for the probability density (Fokker-Planck equation). ${ }^{13}$

However, in our problem we must consider spectra which may not be constant, since we are searching for the one corresponding to equilibrium.

In the case of a stochastic field which is not a white noise, the solution of Eq. (2.2) is not a Markov process. Accordingly, there is no FokkerPlanck equation of the usual type (second-order partial derivatives). However, it is possible to obtain several approximate equations ${ }^{14}$ containing second-order partial derivatives for the probability density in phase space. The basic feature underlying these approximations is the fact that the damping and stochastic forces are small, of order $\tau$ $\left(\equiv 2 e^{2} / 3 m c^{3}\right)$ with respect to the "deterministic unperturbed" Hamiltonian [i.e., $H=\left(m^{2} c^{4}\right.$ $\left.\left.+p^{2} c^{2}\right)^{1 / 2}+V\right]$, which makes it possible to use perturbation methods.

The essential idea of these methods is to switch to an "interaction representation", namely to introduce as new variables a set of "constants of motion" corresponding to the "unperturbed" problem (i.e., without the damping and the stochastic forces). The "initial conditions" provide a very general kind of constants of motion (noticeably, they may be used for any kind of dynamical system, Hamiltonian or not). ${ }^{15}$ Once this change of 
variables has been performed, the remaining forces (damping and stochastic forces) are small and produce only a small diffusion in the space of the new variables. This feature makes it possible to use perturbation methods. Several methods are possible, and the result is a Fokker-Planck-"type" equation for the probability density in phase space. (The reader interested in more technical details may consult Refs. 14-16.)

We may give a qualitative idea of these methods by describing briefly one of them, the Markovian approximation. ${ }^{17}$ We choose an intermediate time scale $\Delta t$, large with respect to the correlation time $t_{c}$ of the stochastic force and small enough to have a small variation in the new variables (constants of motion) that we have introduced before. Then, using this time scale, the process solution of Eqs. (2.2a) and (2.2b) may be approximated by a Markovian process and we may get an approximate Fokker-Planck equation in phase-space variables.

From these Fokker-Planck-type equations, we may obtain a "reduced" Fokker-Planck equation for the stationary density in terms of the constants of motion of the unperturbed system (i.e., without the damping and the stochastic forces). Indeed, since the deterministic orbits are slowly perturbed by the damping and stochastic forces, it is physically intuitive that it will be possible to describe the stochastic motion as a "diffusion of the orbits" rather than a diffusion of the phase points themselves. This reduced Fokker-Planck equation may be obtained by using a "reduction procedure" dev- ised by Haken. ${ }^{18}$ It consists essentially in taking the average over the orbits which are characterized by prescribed values $\left\{h_{\lambda}\right\}$ of the constants of motion of the unperturbed system.

The reduced equation for the stationary density obtained from the different Fokker-Planck-type equations in phase space is unique, which proves the consistency between the different approximations: the same stationary density is obtained in the limit $\tau \rightarrow 0$.

We give now the final result for the reduced Fokker-Planck equation for the stationary density $W_{0}$. The technical details of its obtention may be found in Ref. 19. In a "current" form this equation reads

$$
\sum_{\mu} \frac{\partial}{\partial h_{\mu}}\left[G^{\mu} W_{0}\right]+\sum_{\mu, \nu} \frac{\partial}{\partial h_{\mu}}\left[G^{\mu \nu} \frac{\partial W_{0}}{\partial h_{v}}\right]=0
$$

where $\left\{h_{\mu}\right\}$ are the constants of motion of the deterministic system without the damping.

If we write the stochastic differential equations in the form

$$
\dot{\zeta}_{i}=\mathscr{F}_{i}+\alpha^{2} \mathscr{F}_{i}^{d}+\alpha K_{i}, \quad i=1, \ldots, 6,
$$

where $\mathscr{F}_{i}$ represents the deterministic force, $\alpha^{2} \mathscr{F}_{i}{ }^{d}$ the damping force due to radiation emission, and $\alpha K_{i}$ the force of the random field, verifying $\left\langle K_{i}\right\rangle=0$, the coefficients $G^{\mu}$ and $G^{\mu \nu}$ have the following expressions:

$$
\begin{aligned}
& G^{\mu}=-\alpha^{2} \sum_{j=1}^{6} \mathrm{~T}\left[\mathscr{F}_{j}^{d} \frac{\partial h_{\mu}}{\partial \zeta_{j}}\right]+\mathrm{T}\left[\alpha^{2} \int_{0}^{\infty} d u \sum_{r, l=1}^{6} \frac{\partial h_{\mu}}{\partial \xi_{r}}\left\langle K_{r}(\vec{\xi}, 0) \frac{\partial K_{l}\left(\vec{\xi}^{-u},-u\right.}{\partial \xi_{l}^{-u}}\right\rangle\right], \\
& G^{\mu v}=\alpha^{2} T\left[\int_{0}^{\infty} d u \sum_{r, l=1}^{6} \frac{\partial h_{\mu}}{\partial \xi_{r}} \frac{\partial h_{\mu}}{\partial \xi_{l}^{-u}}\left\langle K_{r}(\vec{\xi}, 0) K_{l}\left(\vec{\xi}^{-u},-u\right)\right\rangle\right] .
\end{aligned}
$$

T represents the following operation on a function $\psi(\vec{\xi})$ of the phase-space variables:

$$
\mathrm{T}[\psi]=\int \psi(\vec{\xi}) d \vec{\xi} \prod_{i=1}^{m} \delta\left(h_{i}-h_{i}(\vec{\xi})\right)
$$

The physical meaning of $T$ is an average over the deterministic orbit, keeping the value of the constants of motion $h_{i}$.

In the case of a multiperiodic system $T$ consists of an integration over the variables $\vec{w}$, since

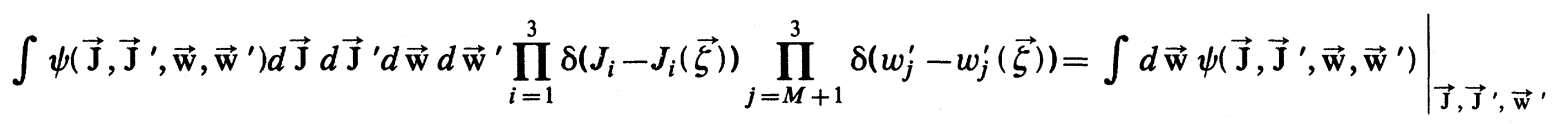

This last expression for $T$ has been used in Eq. (2.10).

In (3.3a) and (3.3b) the notation $\vec{\xi}^{-u}$ means the value of the phase-space coordinates at time $-u$, if they 
are $\vec{\zeta}$ at time 0 , considering only the deterministic motion.

If we compare (3.2) with (2.2), it is easily seen that

$$
\begin{aligned}
& i=1,2,3 \Longrightarrow \zeta_{i}=x_{i}, \quad \mathscr{F}_{i}=\frac{p_{i} c}{\left(p^{2}+m^{2} c^{2}\right)^{1 / 2}}, \mathscr{F}_{i}^{d}=K_{i}=0, \\
& \zeta_{i+3}=p_{i}, \quad \mathscr{F}_{i+3}=\left(\overrightarrow{\mathrm{F}}^{\mathrm{det}}\right)_{i}, \\
& \alpha^{2} \mathscr{F}_{i+3}^{d}=\left(\overrightarrow{\mathrm{F}}^{d}\right)_{i}=\frac{2 e^{2}}{3 c^{3}}\left[\dot{\alpha}_{i}-\frac{1}{c^{2}} \alpha_{\imath} \alpha^{v} v_{i}\right], \\
& \alpha K_{i+3}=F_{i}^{\mathrm{st}}=e\left[E_{i}+\frac{(\overrightarrow{\mathrm{v}} \times \overrightarrow{\mathrm{B}})_{i}}{c}\right] .
\end{aligned}
$$

It should be noted that we have introduced nonsymmetrical diffusion coefficients $G^{\nu \mu} \neq G^{\mu \nu}$. The reason for this is that in the case of a stochastic force with divergence zero $\sum_{e} \partial K_{e} / \partial \zeta_{e}=0$, the contributions of the stochastic and the damping forces are separated. The damping contributes only to the drift term and the stochastic force to the diffusion. This is indeed the case for our stochastic force since

$$
\begin{aligned}
\sum_{l=1}^{6} \frac{\partial K_{l}}{\partial \xi_{l}}=\sum_{i=1}^{3} \frac{\partial}{\partial p_{i}}\left[E_{i}+\frac{(\overrightarrow{\mathbf{v}} \times \overrightarrow{\mathbf{B}})_{i}}{c}\right] & =\sum_{i=1}^{3} \frac{\partial}{\partial p_{i}}\left[\sum_{j, k} \epsilon_{i j k} \frac{v_{j} B_{k}}{c}\right] \\
& =\sum_{k} \frac{B_{k}}{c} \sum_{i j} \epsilon_{i j k} \frac{\partial}{\partial p_{i}}\left[\frac{p_{j}}{\left(p^{2}+m^{2} c^{2}\right)^{1 / 2}}\right] \\
& =\sum_{k} \frac{B_{k}}{c} \sum_{i j} \epsilon_{i j k}\left[\frac{\delta_{i j}}{\left(p^{2}+m^{2} c^{2}\right)^{1 / 2}}-\frac{p_{i} p_{j}}{\left(p^{2}+m^{2} c^{2}\right)^{3 / 2}}\right]=0 .
\end{aligned}
$$

Therefore we get finally the equation (3.1) with the following drift and diffusion coefficients:

$$
\begin{aligned}
& G^{\mu}=-\sum_{j=1}^{3} \mathrm{~T}\left(F_{j}^{d} \frac{\partial h_{\mu}}{\partial p_{j}}\right), \\
& G^{\mu v}=\mathrm{T}\left[\int_{0}^{\infty} d u \sum_{r, l=1}^{3} \frac{\partial h_{\mu}}{\partial p_{r}} \frac{\partial h_{v}}{\partial p_{l}^{-u}}\left\langle F_{r}^{\mathrm{st}}(\overrightarrow{\mathrm{r}}, \overrightarrow{\mathrm{p}}, 0) F_{l}^{\mathrm{st}}\left(\overrightarrow{\mathrm{r}}^{-u}, \overrightarrow{\mathrm{p}}^{-u},-u\right)\right\rangle\right] .
\end{aligned}
$$

\section{FOKKER-PLANCK EQUATION FOR MULTIPERIODIC SYSTEMS AND SOLUTIONS WHICH DEPEND ONLY ON THE ENERGY}

We are going to study the problem of relativistic multiperiodic system interacting with a random radiation. The equilibrium state for such a system is given by the stationary density, which is a solution of a Fokker-Planck-type equation, as stated in Sec. III. If there is a stationary solution for that equation which is normalizable in the phase space, such a solution is unique. ${ }^{20}$ Then, it corresponds to an ergodic process, more technically called recurrent.

Our aim is to show that a spectrum of Rayleigh-Jeans type for the random radiation implies a Maxwell-Boltzmann distribution at equili- brium for the system. For a free particle, the Maxwell-Boltzmann distribution does not depend on position, and it is a constant over space. That distribution is not normalizable and it is not a true probability distribution in phase space. We are then forced to assume that the potential $V(\overrightarrow{\mathrm{r}})$ goes to infinity quickly enough for a function of the form $e^{-V(\vec{r}) / \mathscr{B}_{0}}$ to be integrable. This is fulfilled if it is infinity everywhere except in a finite region, but this is not necessary. A weaker condition is, for example, the existence of a real number $\alpha>0$ such that $V(\overrightarrow{\mathrm{r}}) \geq \mathrm{const} \times r^{\alpha}$ for $r \rightarrow \infty$. Assuming, then, that $V$ behaves adequately, we shall prove that the Maxwell-Boltzmann distribution is a solution of the stationary Fokker-Planck equation, which, therefore, is the searched solution.

We begin defining the funtionals 


$$
\begin{aligned}
& G_{1}(\varphi)=-\sum_{j=1}^{3} \mathrm{~T}\left[F_{j}^{d} \frac{\partial \varphi}{\partial p_{j}}\right] \\
& \bar{G}_{2}(\varphi, \psi)=\mathrm{T}\left[\int_{0}^{\infty} d u \sum_{r, l=1}^{3} \frac{\partial \varphi}{\partial p_{r}} \frac{\partial \psi}{\partial p_{l}^{-u}}\left\langle F_{r}^{\mathrm{st}}(\overrightarrow{\mathrm{r}}, \overrightarrow{\mathrm{p}}, 0) F_{l}^{\mathrm{st}}\left(\overrightarrow{\mathrm{r}}^{-u}, \overrightarrow{\mathrm{p}}^{-u},-u\right)\right\rangle\right]
\end{aligned}
$$

for any two functions $\varphi$ and $\psi$. In our problem of a multiperiodic motion, these functions will be the action constants $J_{1}, J_{2}, J_{3}$ and angle constants $w_{M+1}, \ldots, w_{3}$ (i.e., $\overrightarrow{\mathbf{J}}, \overrightarrow{\mathbf{J}}$ ', and $\overrightarrow{\mathbf{w}}^{\prime}$ according to our notation of Sec. II C), that is, $6-M$ constants. Now we shall search for solutions of the Fokker-Planck equation of the form

$$
W_{0}=W_{0}[\mathscr{E}(\overrightarrow{\mathrm{r}}, \overrightarrow{\mathrm{p}})]
$$

i.e., depending on $\overrightarrow{\mathbf{r}}$ and $\overrightarrow{\mathrm{p}}$ only through the energy. As $\mathscr{E}$ depends only on $\overrightarrow{\mathbf{J}}$ and not on $\overrightarrow{\mathbf{J}}^{\prime}$, nor $\overrightarrow{\mathbf{w}}^{\prime}$ (see Sec. II C), then the second term of the Fokker-Planck equation (3.1), includes only a sum for $v=1, \ldots, M$. Also

$$
\frac{\partial W_{0}}{\partial J_{i}}=W_{0}^{\prime} \omega_{i}
$$

Therefore in (3.4a) and (3.4b) appear only the functions $G^{\mu v}$ corresponding to $h_{v}=J_{v}, v=1, \ldots, M$. Besides,

$$
G^{\mu v}=\bar{G}_{2}\left(h_{\mu}, h_{v}\right) .
$$

So we can define

$$
\begin{aligned}
G_{2}(\varphi) & =\sum_{v=1}^{M} \bar{G}_{2}\left(\varphi, J_{v}\right) \omega_{v} \\
& =\mathrm{T}\left[\int_{0}^{\infty} d u \sum_{v=1}^{M} \sum_{r, l=1}^{3} \frac{\partial \varphi}{\partial p_{r}} \frac{\partial J_{v}}{\partial p_{l}^{-u}} \omega_{v}\left\langle F_{r}^{\mathrm{st}}(\overrightarrow{\mathrm{r}}, \overrightarrow{\mathrm{p}}, 0) F_{l}^{\mathrm{st}}\left(\overrightarrow{\mathrm{r}}^{-u}, \overrightarrow{\mathrm{p}}^{-u},-u\right)\right\rangle\right]
\end{aligned}
$$

and the Fokker-Planck equation can be written in the form

$$
\begin{aligned}
0= & \sum_{i=1}^{M} \frac{\partial}{\partial J_{i}}\left[G_{1}\left(J_{i}\right) W_{0}+G_{2}\left(J_{i}\right) W_{0}^{\prime}\right]+\sum_{i=M+1}^{3} \frac{\partial}{\partial J_{i}^{\prime}}\left[G_{1}\left(J_{i}^{\prime}\right) W_{0}+G_{2}\left(J_{i}^{\prime}\right) W_{0}^{\prime}\right] \\
& +\sum_{i=M+1}^{3} \frac{\partial}{\partial w_{i}^{\prime}}\left[G_{1}\left(w_{i}^{\prime}\right) W_{0}+G_{2}\left(w_{i}^{\prime}\right) W_{0}^{\prime}\right] .
\end{aligned}
$$

\section{CALCULATION OF THE COEFFICIENTS OF THE FOKKER-PLANCK EQUATION}

We begin with the calculation of the general form of the functionals $G_{1}$ and $G_{2}$ :

\section{A. Calculation of $\boldsymbol{G}_{1}(\varphi)$}

Taking into account Eqs. (4.1a) and (2.2a) and $(2.2 b)$, we have

$$
\begin{aligned}
G_{1}(\varphi) & =-\mathrm{T}\left[\overrightarrow{\mathrm{F}}^{d} \cdot \vec{\varphi}_{p}\right] \\
& =-\frac{2 e^{2}}{3 c^{3}} \int d \overrightarrow{\mathrm{w}}\left[\left(\dot{\vec{\alpha}}-\frac{1}{c^{2}} \alpha^{\lambda} \alpha_{\lambda} \overrightarrow{\mathrm{v}}\right) \cdot \vec{\varphi}_{p}\right],
\end{aligned}
$$

where we have written $\vec{\varphi}_{p}$ for $\vec{\nabla}_{p} \varphi$. On the other hand, the following relations hold ${ }^{8}$ :

$$
\begin{aligned}
& \alpha^{\mu}=\left(\alpha^{0}, \vec{\alpha}\right)=\left(\gamma^{4} \frac{\overrightarrow{\mathrm{v}} \cdot \overrightarrow{\mathrm{a}}}{c}, \gamma^{2} \vec{\alpha}+\gamma^{4} \frac{\overrightarrow{\mathrm{v}} \cdot \overrightarrow{\mathrm{a}}}{c^{2}} \overrightarrow{\mathrm{v}}\right), \\
& \alpha^{\lambda} \alpha_{\lambda}=\gamma^{4} a^{2}+\gamma^{6}\left(\frac{\overrightarrow{\mathrm{v}} \cdot \overrightarrow{\mathrm{a}}}{c}\right)^{2} .
\end{aligned}
$$

In order to show that the Maxwell-Boltzmann distribution is a solution of the Fokker-Planck equation, it is convenient to write $G_{1}$ and $G_{2}$ in similar form. In Eq. (4.2) it is seen that the coefficient $G_{2}$ is written in terms of the correlation function of the random field. If we perform the 
Fourier transform of that, it results in an expression related to the spectral density of the random field. Therefore, it is convenient to write $G_{1}$ also in terms of frequencies. It is easy to see, however, that this cannot be made directly from the expression $\overrightarrow{\mathrm{F}}^{d} \cdot \vec{\varphi}_{p}$ which is averaged over an orbit in $G_{1}$. Actually, we need new expressions for (4.1).

In order to see more easily the way to do that, let us consider the particular case in which $\varphi$ is the energy (which is not true in general). Then

$$
\frac{\partial \mathscr{E}}{\partial p_{j}}=v_{j}
$$

and

$$
G_{1}(\mathscr{E})=-\int d \overrightarrow{\mathrm{w}} \overrightarrow{\mathrm{F}}^{d} \cdot \overrightarrow{\mathrm{v}},
$$

that is, $G_{1}(\mathscr{C})$ is the average energy lost per unit time as a consequence of radiation by the charged particle. On the other hand, we know that this energy is given by the flux of the Poynting vector $S$ through a spherical surface at infinity. With the notation of Ref. 21 we have

$$
|\Delta \mathscr{E}|=\lim _{r \rightarrow \infty} \lim _{T \rightarrow \infty} \frac{1}{T} \int_{0}^{T} d t \int d \Omega\left(R^{2} \overrightarrow{\mathrm{S}} \cdot \hat{n}\right)_{\mathrm{ret}},
$$

where

$$
\overrightarrow{\mathbf{R}}(\overrightarrow{\mathbf{r}}, t)=\overrightarrow{\mathbf{r}}-\vec{\xi}(t), \hat{n}=\frac{\overrightarrow{\mathbf{R}}}{|\overrightarrow{\mathbf{R}}|},
$$

$\vec{\xi}(t)$ is the solution of the equations of motion with only the deterministic forces and the subscript "ret" means that the quantities must be considered at the retarded time. If we take into account that only the "acceleration fields" give rise to a loss of energy by the charge, ${ }^{8}$ it results in

$$
G_{1}(\mathscr{E})=-(2 \pi)^{M}|\Delta \mathscr{E}|=-\lim _{r \rightarrow \infty} \lim _{T \rightarrow \infty} \frac{1}{T} \int_{0}^{T} d t \int d \Omega\left|\overrightarrow{\mathrm{g}}^{r}\right|_{\mathrm{ret}}^{2}(2 \pi)^{M} \frac{e^{2}}{4 \pi c}
$$

where

$$
\overrightarrow{\mathrm{g}}^{r}=\frac{\hat{n} \times[(\hat{n}-\vec{\beta}) \times \dot{\vec{\beta}}]}{(1-\vec{\beta} \cdot \hat{n})^{3}} \text { and } \vec{\beta}=\overrightarrow{\mathrm{v}} / c .
$$

$$
\hat{n}=\frac{-c}{|\overrightarrow{\mathrm{r}}-\vec{\xi}(t)|}(\vec{\beta}-\hat{n} \cdot \vec{\beta} \hat{n}) \rightarrow 0
$$

and therefore

$$
\overrightarrow{\mathrm{g}}^{r} \underset{r \rightarrow \infty}{\rightarrow} \overrightarrow{\mathrm{g}}^{\infty}=\frac{\hat{r}^{0} \times\left[\left(\hat{r}^{0}-\vec{\beta}\right) \times \dot{\vec{\beta}}\right]}{\left(1-\vec{\beta} \cdot \hat{r}^{0}\right)^{3}} .
$$

Then, it can be shown by direct calculation that

$$
\begin{aligned}
\overrightarrow{\mathrm{g}}^{\infty} & =\frac{1}{\left(1-\vec{\beta} \cdot \hat{r}^{0}\right)} \frac{d}{d t}\left[\frac{\hat{r}^{0} \times\left(\hat{r}^{0} \times \vec{\beta}\right)}{\left(1-\vec{\beta} \cdot \hat{r}^{0}\right)}\right] \\
& =\lim _{r \rightarrow \infty} \frac{1}{1-\vec{\beta} \cdot \hat{n}} \frac{d}{d t}\left[\frac{\hat{n} \times(\hat{n} \times \vec{\beta})}{1-\vec{\beta} \cdot \hat{n}}\right] .
\end{aligned}
$$

Let us define $\hat{n} \rightarrow \hat{r}^{0}=\vec{r} / r$,

$$
\begin{aligned}
& \overrightarrow{\mathrm{h}}_{\varphi}(\overrightarrow{\mathrm{r}}, t)=\frac{1}{1-\vec{\beta} \cdot \hat{n}} \frac{\partial}{\partial t}\left[\frac{\hat{n}(\overrightarrow{\mathrm{r}}, t) \times\left\{[\hat{n}(\overrightarrow{\mathrm{r}}, t)-\vec{\beta}(t)] \times \vec{\varphi}_{p}(t)\right\}}{1-\vec{\beta}(t) \cdot \hat{n}(\overrightarrow{\mathrm{r}}, t)}\right], \\
& \overrightarrow{\mathbf{g}}(\overrightarrow{\mathrm{r}}, t)=\frac{1}{1-\vec{\beta} \cdot \hat{n}} \frac{\partial}{\partial t}\left[\frac{\hat{n}(\overrightarrow{\mathrm{r}}, t) \times[\hat{n}(\overrightarrow{\mathrm{r}}, t) \times \vec{\beta}(t)]}{1-\vec{\beta}(t) \cdot \hat{n}(\overrightarrow{\mathrm{r}}, t)}\right] .
\end{aligned}
$$

If

$\varphi \equiv \mathscr{B}$,
then $\overrightarrow{\mathrm{h}}_{\mathscr{\mathscr { C }}} \underset{r \rightarrow \infty}{\rightarrow} c \overrightarrow{\mathrm{g}}^{\infty}$

$\overrightarrow{\mathrm{g}} \underset{r \rightarrow \infty}{\rightarrow} \overrightarrow{\mathrm{g}}^{\infty}$
After that, we shall prove the following:

$$
\begin{aligned}
G_{1}(\varphi)= & \frac{e^{2}}{4 \pi c^{2}}(2 \pi)^{M} \lim _{r \rightarrow \infty} \lim _{T \rightarrow \infty} \frac{1}{T} \\
& \times \int_{0}^{T} d t \int d \Omega\left[\overrightarrow{\mathrm{h}}_{\varphi}(\overrightarrow{\mathrm{r}}, t) \cdot \overrightarrow{\mathrm{g}}(\overrightarrow{\mathrm{r}}, t)\right]_{\mathrm{ret}},
\end{aligned}
$$


which is the desired generalization of (5.3).

From (5.5) we shall obtain the expression of $G_{1}(\varphi)$ in terms of frequencies by expanding $\overrightarrow{\mathrm{h}}_{\varphi}$ and $\vec{g}$ in Fourier series. But before that we first prove Eq. (5.5). To do this, we need three relations, which are proven below.

(1) The first relation is

$$
G_{1}(\varphi)=\frac{2 e^{2}}{3 c^{3}} T[\Lambda]
$$

with

$$
\begin{aligned}
\Lambda \equiv & \left(\dot{\vec{\varphi}}_{p} \cdot \dot{\vec{\beta}}\right) \gamma^{2}+\left(\dot{\vec{\varphi}}_{p} \cdot \vec{\beta}\right) \gamma^{4}(\vec{\beta} \cdot \dot{\vec{\beta}}) \\
& +\left(\vec{\beta} \cdot \vec{\varphi}_{p}\right)\left[\gamma^{4} \dot{\vec{\beta}}^{2}+\gamma^{6}(\vec{\beta} \cdot \dot{\vec{\beta}})^{2}\right]
\end{aligned}
$$

In order to prove that, it is enough to combine (5.1) with (5.2) and to apply the property (2.9) to the first term in the integrand,

$$
\begin{aligned}
\mathrm{T}\left[\dot{\vec{\alpha}} \cdot \vec{\varphi}_{p}\right]= & (2 \pi)^{M} \lim _{T \rightarrow \infty} \frac{1}{T} \int_{0}^{T} d t\left(\dot{\vec{\alpha}} \cdot \vec{\varphi}_{p}\right) \\
& (2 \pi)^{M} \lim _{T \rightarrow \infty} \frac{1}{T}\left[\left.\vec{\alpha} \cdot \vec{\varphi}_{p}\right|_{0} ^{T}-\int_{0}^{T} \vec{\alpha} \cdot \dot{\vec{\varphi}}_{p} d t\right] \\
& =-\mathrm{T}\left[\vec{\alpha} \cdot \dot{\vec{\varphi}}_{p}\right]
\end{aligned}
$$

because, by the nature of the motion, $\vec{\alpha}$ and $\vec{\varphi}_{p}$ are bounded in time. Finally, $\overrightarrow{\mathrm{a}}=c \vec{\beta}$ and replacing (5.2) we see that (5.6) follows immediately.

(2) Let $f(\overrightarrow{\mathrm{r}}, \overrightarrow{\mathrm{p}})$ be a continuous function in phase space such that $\lim _{r \rightarrow \infty} f(\overrightarrow{\mathrm{r}}, \overrightarrow{\mathrm{p}})=f(\infty, \Omega, \overrightarrow{\mathrm{p}})$ exists

( $\Omega$ is the solid angle). Then

$$
\begin{aligned}
\lim _{r \rightarrow \infty} \int d \overrightarrow{\mathrm{w}} \int d \Omega f(r, \Omega, \overrightarrow{\mathrm{p}}) \\
=\int d \overrightarrow{\mathrm{w}} \int d \Omega f(\infty, \Omega, \overrightarrow{\mathrm{p}}) .
\end{aligned}
$$

This is true because the integrals have finite limits and the expression under the integral sign is continuous (Ref. 22).

(3) Let $f$ be a continuous and bounded function in $R^{3} \times \Gamma(\Gamma \equiv$ phase space $)$; then

$$
\begin{aligned}
& \lim _{T \rightarrow \infty} \frac{1}{T} \int_{0}^{T} d t f(\overrightarrow{\mathrm{r}}, \vec{\xi}(t), \vec{\beta}(t))_{\text {ret }} \\
& \quad=\lim _{T \rightarrow \infty} \frac{1}{T} \int_{0}^{T} d t f(\overrightarrow{\mathrm{r}}, \vec{\xi}(t), \vec{\beta}(t))(1-\vec{\beta} \cdot \hat{n}) .
\end{aligned}
$$

In order to prove this, let us make in the first integral the change

$$
t^{\prime}=t_{\text {ret }}(\overrightarrow{\mathrm{r}}, t)
$$

[remember that $t_{\text {ret }}$ verifies

$$
\begin{aligned}
c\left(t-t_{\text {ret }}\right) & =\left|\overrightarrow{\mathbf{R}}\left(\overrightarrow{\mathrm{r}}, t_{\text {ret }}\right)\right| \\
& \left.=\left|\overrightarrow{\mathrm{r}}-\vec{\xi}\left(t_{\text {ret }}\right)\right|\right]
\end{aligned}
$$

so that

$$
d t=d t^{\prime}\left[1-\vec{\beta}\left(t^{\prime}\right) \cdot \hat{n}\left(\overrightarrow{\mathrm{r}}, t^{\prime}\right)\right] .
$$

Then

$$
\begin{aligned}
& \int_{0}^{T} d t f(\overrightarrow{\mathrm{r}}, \vec{\xi}(t), \vec{\beta}(t))_{\mathrm{ret}} \\
&=\int_{t_{\mathrm{ret}}(\overrightarrow{\mathrm{r}}, 0)}^{t_{\mathrm{ret}}(\overrightarrow{\mathrm{r}}, T)} f\left(\overrightarrow{\mathrm{r}}, \vec{\xi}\left(t^{\prime}\right), \vec{\beta}\left(t^{\prime}\right)\right)(1-\vec{\beta} \cdot \hat{n}) d t^{\prime}
\end{aligned}
$$

Now, as $\vec{\xi}(t)$ is constrained within a finite region due to the condition on the potential (region whose limits will depend on the constants of the motion), the results is that there exists some constant $R_{M}$ such that $|\vec{\xi}(t)|<R_{M}$ for any $t$ and, therefore,

$$
c\left|t-t_{\text {ret }}\right|=\left|\overrightarrow{\mathbf{R}}\left(\overrightarrow{\mathrm{r}}, t_{\text {ret }}\right)\right|<r+R_{M} \text {. }
$$

As a consequence,

$$
\begin{aligned}
\left|\int_{t_{\mathrm{ret}}(\overrightarrow{\mathrm{r}}, 0)}^{0} f\left(\overrightarrow{\mathrm{r}}, \vec{\xi}\left(t^{\prime}\right), \vec{\beta}\left(t^{\prime}\right)\right)(1-\vec{\beta} \cdot \hat{n}) d t^{\prime}\right| & \leq \int_{t_{\mathrm{ret}}(\overrightarrow{\mathrm{r}}, 0)}^{0}\left|f\left(\overrightarrow{\mathrm{r}}, \vec{\xi}\left(t^{\prime}\right), \vec{\beta}\left(t^{\prime}\right)\right)(1-\vec{\beta} \cdot \hat{n})\right| d t^{\prime} \\
& \leq \int_{-\left(r+R_{M} / c\right)}^{0}\left|f\left(\overrightarrow{\mathrm{r}}, \vec{\xi}\left(t^{\prime}\right), \vec{\beta}\left(t^{\prime}\right)\right)(1-\vec{\beta} \cdot \hat{n})\right| d t^{\prime}
\end{aligned}
$$

So that, after dividing by $T$ and taking the limit $T \rightarrow \infty$ the corresponding term is zero. The same is true for the upper limit of the integral on the right-hand side of $(5.10), t_{\text {ret }}(\vec{r}, T)$, so that we have finally

$$
\lim _{T \rightarrow \infty} \frac{1}{T} \int_{t_{\text {ret }}(\vec{r}, 0)}^{t_{\text {ret }}(\vec{r}, T)} f\left(\overrightarrow{\mathrm{r}}, \vec{\xi}\left(t^{\prime}\right), \vec{\beta}\left(t^{\prime}\right)\right)(1-\vec{\beta} \cdot \hat{n}) d t^{\prime}=\lim _{T \rightarrow \infty} \frac{1}{T} \int_{0}^{T} f(\vec{r}, \vec{\xi}(t), \vec{\beta}(t))(1-\vec{\beta} \cdot \hat{n}) d t
$$


which proves Eq. (5.9).

After that, we may go to the proof of Eq. (5.5). If we apply (5.9) to the function

$$
\int d \Omega \overrightarrow{\mathrm{h}}_{\varphi} \cdot \overrightarrow{\mathrm{g}},
$$

we obtain

$$
\begin{aligned}
& \lim _{r \rightarrow \infty} \lim _{T \rightarrow \infty} \frac{1}{T} \int_{0}^{T} d t \int d \Omega\left[\overrightarrow{\mathrm{h}}_{\varphi} \cdot \overrightarrow{\mathrm{g}}\right]_{\mathrm{ret}} \\
& \quad=\lim _{r \rightarrow \infty} \frac{1}{(2 \pi)^{M}} T\left[\int d \Omega\left(\overrightarrow{\mathrm{h}}_{\varphi} \cdot \overrightarrow{\mathrm{g}}\right)(1-\vec{\beta} \cdot \hat{n})\right],
\end{aligned}
$$

where we have used (2.9) and (2.10). Now, taking Eqs. (5.8) and (2.9) we have

$$
\begin{aligned}
\lim _{r \rightarrow \infty} \lim _{T \rightarrow \infty} & \frac{1}{T} \int_{0}^{T} d t \int d \Omega\left[\overrightarrow{\mathrm{h}}_{\varphi} \cdot \overrightarrow{\mathrm{g}}\right]_{\mathrm{ret}} \\
= & \frac{1}{(2 \pi)^{M}} \int d \overrightarrow{\mathrm{w}} \int d \Omega\left(\overrightarrow{\mathrm{h}}_{\varphi}^{\infty} \cdot \overrightarrow{\mathrm{g}}^{\infty}\right)\left(1-\vec{\beta} \cdot \hat{r}^{0}\right)
\end{aligned}
$$

where, as $\hat{n} \underset{r \rightarrow \infty}{\rightarrow} \hat{r}^{0}$ and $\hat{\hat{n}} \underset{r \rightarrow \infty}{\rightarrow} 0$,

$$
\begin{aligned}
& \overrightarrow{\mathrm{h}}_{\varphi}^{\infty}=\frac{1}{1-\vec{\beta} \cdot \hat{r}^{0}} \frac{d}{d t}\left[\frac{\hat{r}^{0} \times\left[\left(\hat{r}^{0}-\vec{\beta}\right) \times \vec{\varphi}_{p}\right]}{1-\vec{\beta} \cdot \hat{r}^{0}}\right], \\
& \overrightarrow{\mathrm{g}}^{\infty}=\frac{1}{1-\vec{\beta} \cdot \hat{r}^{0}} \frac{d}{d t}\left[\frac{\hat{r}^{0} \times\left(\hat{r}^{0} \times \vec{\beta}\right)}{1-\vec{\beta} \cdot \hat{r}^{0}}\right]
\end{aligned}
$$

In (5.11) the solid angle integration is straightforward, although lengthy. Details are given in Appendix A. The result is

$$
\int d \Omega\left(\overrightarrow{\mathrm{h}}_{\varphi}^{\infty} \cdot \overrightarrow{\mathrm{g}}^{\infty}\right)\left(1-\vec{\beta} \cdot \hat{r}^{0}\right)=\frac{8 \pi}{3} \Lambda .
$$

Taking into account (2.9), (2.10), and (5.6) we obtain finally Eq. (5.5).

\section{B. Frequency analysis of $G_{1}(\varphi)$}

We must perform a frequency analysis of the vectors $\overrightarrow{\mathrm{h}}_{\varphi}$ and $\overrightarrow{\mathrm{g}}$. We shall start proving that the only frequencies involved are of the form

$$
\omega=\vec{\omega}_{0} \cdot \overrightarrow{\mathrm{n}}=\sum_{i=1}^{M} \omega_{i} n_{i}
$$

with $n$ integer.

We shall consider only $\overrightarrow{\mathrm{g}}$, the proof for $\overrightarrow{\mathrm{h}}_{\varphi}$ being similar. We take the Fourier transform of $\left.\overrightarrow{\mathrm{g}}\right|_{\text {ret }}$

$$
\overrightarrow{\mathscr{G}}(\omega)=\left.\frac{1}{2 \pi} \int_{-\infty}^{+\infty} \overrightarrow{\mathrm{g}}\right|_{\mathrm{ret}} e^{-i \omega t} d t .
$$

Then, we perform the change $t^{\prime}=t_{\text {ret }}$ so that, $|\overrightarrow{\mathrm{r}}-\vec{\xi}(t)|$ being bounded, $t= \pm \infty \Longrightarrow t^{\prime}= \pm \infty$ and, therefore,

$$
\begin{gathered}
\overrightarrow{\mathscr{G}}(\omega)=\frac{1}{2 \pi} \int_{-\infty}^{+\infty} \overrightarrow{\mathbf{g}}\left(t^{\prime}\right) e^{-i \omega t\left(t^{\prime}\right)} \\
\times \frac{d t}{d t^{\prime}} d t^{\prime} .
\end{gathered}
$$

Now, from the definition of $t_{\text {ret }}$ the result is

$$
t=t^{\prime}+\frac{\left|\overrightarrow{\mathrm{r}}-\vec{\xi}\left(t^{\prime}\right)\right|}{c}
$$

so that

$$
\overrightarrow{\mathscr{G}}(\omega)=\frac{1}{2 \pi} \int_{-\infty}^{+\infty} \overrightarrow{\mathrm{g}}\left(t^{\prime}\right) \exp \left\{-i \omega\left[t^{\prime}+\frac{\left|\overrightarrow{\mathrm{r}}-\vec{\xi}\left(t^{\prime}\right)\right|}{c}\right]\right\}(1-\vec{\beta} \cdot \hat{n}) d t^{\prime} .
$$

Note that all quantities under the integral are calculated at time $t^{\prime}$ (except $\vec{r}$, which is fixed). Let

$$
\vec{\eta}=\overrightarrow{\mathrm{g}}\left(t^{\prime}\right) \exp \left[-i \omega \frac{\left.\mid \overrightarrow{\mathrm{r}}-\vec{\xi}\left(t^{\prime}\right)\right\rfloor}{c}\right]\left[1-\vec{\beta}\left(t^{\prime}\right) \cdot \hat{n}\left(\overrightarrow{\mathrm{r}}, t^{\prime}\right)\right],
$$

$\vec{\eta}$ depends on $t^{\prime}$ through $\vec{\xi}$ and $\vec{\beta}$, which are periodic functions of the $w_{i}$ and, therefore, $\vec{\eta}$ will be also a periodic function of the angle variables. Then, it is possible to construct a Fourier series expansion in the form

$$
\begin{aligned}
\vec{\eta}=\vec{\eta}\left(\overrightarrow{\mathrm{r}}, \overrightarrow{\mathrm{w}}\left(t^{\prime}\right)\right) & =\sum_{\overrightarrow{\mathrm{n}}} \vec{\eta}_{\overrightarrow{\mathrm{n}}}(\overrightarrow{\mathrm{r}}) e^{i \overrightarrow{\mathrm{n}} \cdot \overrightarrow{\mathrm{w}}\left(t^{\prime}\right)} \\
& =\sum_{\overrightarrow{\mathrm{n}}} \vec{\eta}_{\overrightarrow{\mathrm{n}}}(\overrightarrow{\mathrm{r}}) e^{i \overrightarrow{\mathrm{n}} \cdot \overrightarrow{\mathrm{w}}_{0}} e^{i \overrightarrow{\mathrm{n}} \cdot \vec{\omega}_{0} t^{\prime}}
\end{aligned}
$$

Putting these expressions in (5.15)

$$
\begin{aligned}
\overrightarrow{\mathscr{G}}(\omega) & =\frac{1}{2 \pi} \int_{-\infty}^{+\infty} \sum_{\overrightarrow{\mathrm{n}}} \vec{\eta}_{\overrightarrow{\mathrm{n}}}(\overrightarrow{\mathrm{r}}) e^{i \overrightarrow{\mathrm{n}} \cdot \overrightarrow{\mathrm{w}}_{0}} e^{i \overrightarrow{\mathrm{n}} \cdot \vec{\omega}_{0} t^{\prime}} e^{-i w t^{\prime}} d t^{\prime} \\
& =\sum_{\overrightarrow{\mathrm{n}}} \vec{\eta}_{\overrightarrow{\mathrm{n}}}(\overrightarrow{\mathrm{r}}) e^{i \overrightarrow{\mathrm{n}} \cdot \overrightarrow{\mathrm{w}}_{0}} \delta\left(\overrightarrow{\mathrm{n}} \cdot \vec{\omega}_{0}-\omega\right) .
\end{aligned}
$$

If we perform now the inverse Fourier transform in (5.14), taking (5.16) into account, 


$$
\begin{aligned}
\left.\overrightarrow{\mathrm{g}}\right|_{\mathrm{ret}}=\int_{-\infty}^{+\infty} \overrightarrow{\mathscr{G}}(\omega) e^{i \omega t} d \omega & =\sum_{\overrightarrow{\mathrm{n}}} \vec{\eta}_{\overrightarrow{\mathrm{n}}}(\overrightarrow{\mathrm{r}}) e^{i \overrightarrow{\mathrm{n}} \cdot \overrightarrow{\mathrm{w}}} e^{i \overrightarrow{\mathrm{n}} \cdot \vec{\omega}_{0} t} \\
& =\sum_{\overrightarrow{\mathrm{n}}} \vec{\eta}_{\overrightarrow{\mathrm{n}}}(\overrightarrow{\mathrm{r}}) e^{i \overrightarrow{\mathrm{n}} \cdot \overrightarrow{\mathrm{w}}(t)}
\end{aligned}
$$

Similarly, for $\overrightarrow{\mathrm{h}}_{\varphi} \mid$ ret we obtain

$$
\left.\overrightarrow{\mathrm{h}}_{\varphi}\right|_{\text {ret }}=\sum_{\overrightarrow{\mathrm{n}}} \vec{\mu}_{\varphi, \overrightarrow{\mathrm{n}}}(\overrightarrow{\mathrm{r}}) e^{i \overrightarrow{\mathrm{n}} \cdot \overrightarrow{\mathrm{w}}(t)}
$$

Note that it is the time $t$, and not the retarded time, which appears in $(5.17 \mathrm{a})$ and $(5.17 \mathrm{~b})$, in spite of the fact that $\overrightarrow{\mathrm{g}}$ and $\overrightarrow{\mathrm{h}}_{\varphi}$ have been calculated at the retarded time.

The next step is to show that, as in $G_{1}(\varphi)$, it is made a time average, each frequency contributing separately, i.e.,

$$
G_{1}(\varphi)=\sum_{\overrightarrow{\mathrm{n}}} G_{1, \overrightarrow{\mathrm{n}}}
$$

where $G_{1, \overrightarrow{\mathrm{n}}}$ means the same expression as $G_{1}$ but putting, instead of $\left.\overrightarrow{\mathrm{h}}_{\varphi}\right|_{\text {ret }}$ and $\left.\overrightarrow{\mathrm{g}}\right|_{\text {ret }}$, the corresponding Fourier components. This is equivalent to saying that each plane wave contributes independently of the others to the value of the coefficient $G_{1}(\varphi)$ in the same way that the radiated energy, when averaged over the time, is equal to the sum of energies carried by the different plane waves. We shall prove then the following:

$$
G_{1}(\varphi)=\frac{e^{2}}{4 \pi c^{2}}(2 \pi)^{M} \lim _{r \rightarrow \infty} \sum_{\overrightarrow{\mathrm{n}}} \int d \Omega\left(\vec{\mu}_{\varphi, \overrightarrow{\mathrm{n}}} \cdot \vec{\eta}_{-\overrightarrow{\mathrm{n}}}\right) .
$$

In fact, from Eqs. (5.17a) and (5.17b) and (5.5), we obtain

$$
\begin{aligned}
& G_{1}(\varphi)= \frac{e^{2}}{4 \pi c^{2}}(2 \pi)^{M} \lim _{r \rightarrow \infty} \lim _{T \rightarrow \infty} \frac{1}{T} \\
& \times \int_{0}^{T} d t \int d \Omega \sum_{\overrightarrow{\mathrm{n}}} \sum_{\overrightarrow{\mathrm{m}}} \vec{\mu}_{\varphi, \overrightarrow{\mathrm{n}}} \cdot \vec{\eta}_{\overrightarrow{\mathrm{m}}} \\
& \\
& \times e^{i \overrightarrow{\mathrm{n}} \cdot \overrightarrow{\mathrm{w}}(t)} e^{i \overrightarrow{\mathrm{m}} \cdot \overrightarrow{\mathrm{w}}(t)},
\end{aligned}
$$

which after performing the time integral, leads to Eq. (5.18).

Now, we must calculate explicitly the Fourier components of $\overrightarrow{\mathrm{h}}_{\varphi}$ and $\overrightarrow{\mathrm{g}}$ in order to introduce them into (5.18). Inverting the relations $(5.17 \mathrm{a})$ and $(5.17 \mathrm{~b})$ we have

$$
\begin{aligned}
& \vec{\eta}_{\overrightarrow{\mathrm{n}}}(\overrightarrow{\mathrm{r}})=\left.\lim _{T \rightarrow \infty} \frac{1}{T} \int_{0}^{T} d t \overrightarrow{\mathrm{g}}\right|_{\mathrm{ret}} e^{-i \overrightarrow{\mathrm{n}} \cdot \overrightarrow{\mathrm{w}}(t)} \\
& \vec{\mu}_{\varphi, \overrightarrow{\mathrm{n}}}(\overrightarrow{\mathrm{r}})=\left.\lim _{T \rightarrow \infty} \frac{1}{T} \int_{0}^{T} d t \overrightarrow{\mathrm{h}}_{\varphi}\right|_{\mathrm{ret}} e^{-i \overrightarrow{\mathrm{n}} \cdot \overrightarrow{\mathrm{w}}(t)}
\end{aligned}
$$

Consider (5.19a). We make the same change as before, namely $t^{\prime}=t_{\text {ret }}(t)$, which gives

$$
\begin{array}{r}
\vec{\eta}_{\overrightarrow{\mathrm{n}}}(\overrightarrow{\mathrm{r}})=\lim _{T \rightarrow \infty} \frac{1}{T} \int_{0}^{T} d t^{\prime} e^{-i \overrightarrow{\mathrm{n}} \cdot \overrightarrow{\mathrm{w}}} 0 e^{-i \overrightarrow{\mathrm{n}} \cdot \vec{\omega}_{0} t\left(t^{\prime}\right)} \\
\times \overrightarrow{\mathrm{g}}\left(t^{\prime}\right)\left[1-\vec{\beta}\left(t^{\prime}\right) \cdot \hat{n}\left(\overrightarrow{\mathrm{r}}, t^{\prime}\right)\right]
\end{array}
$$

after several steps similar to the ones leading to Eq. (5.9). Now

$$
(1-\vec{\beta} \cdot \hat{n}) \overrightarrow{\mathrm{g}}=\frac{d}{d t} \frac{\hat{n} \times(\hat{n} \times \vec{\beta})}{1-\vec{\beta} \cdot \hat{n}},
$$

which, after an integration by parts, leads to

$$
\begin{aligned}
\vec{\eta}_{\overrightarrow{\mathrm{n}}}=\lim _{T \rightarrow \infty}[ & \left.\frac{1}{T}\left[\frac{\hat{n} \times(\hat{n} \times \vec{\beta})}{1-\vec{\beta} \cdot \hat{n}} e^{-i \overrightarrow{\mathrm{n}} \cdot \overrightarrow{\mathrm{w}}_{0}-i \overrightarrow{\mathrm{n}} \cdot \vec{\omega}_{0} t\left(t^{\prime}\right)}\right)\right|_{0} ^{T} \\
& \left.-\frac{1}{T} \int_{0}^{T} d t^{\prime} \frac{\hat{n} \times(\hat{n} \times \vec{\beta})}{1-\vec{\beta} \cdot \hat{n}} \frac{d}{d t^{\prime}}\left(e^{-i \overrightarrow{\mathrm{n}} \cdot \overrightarrow{\mathrm{w}}_{0}-i \overrightarrow{\mathrm{n}} \cdot \vec{\omega}_{0} t\left(t^{\prime}\right)}\right)\right]
\end{aligned}
$$

Again, all quantities are calculated in $t^{\prime}$. The first term in the sum is zero for $T \rightarrow \infty$ for the quantity within brackets is bounded. Then

$$
\begin{aligned}
\vec{\eta}_{\overrightarrow{\mathrm{n}}} & =\lim _{T \rightarrow \infty}\left[-\frac{1}{T}\right] \int_{0}^{T} d t^{\prime} \frac{\hat{n} \times(\hat{n} \times \beta)}{1-\vec{\beta} \cdot \hat{n}}\left(e^{-i \hat{n} \cdot \overrightarrow{\mathrm{w}}_{0}-i \overrightarrow{\mathrm{n}} \cdot \vec{\omega}_{0} t\left(t^{\prime}\right)}\right)\left(-i \overrightarrow{\mathrm{n}}^{-\vec{\omega}_{0}}\right)(1-\vec{\beta} \cdot \hat{n}) \\
& =\lim _{T \rightarrow \infty} i \overrightarrow{\mathrm{n}} \cdot \vec{\omega}_{0} e^{-i \overrightarrow{\mathrm{n}} \cdot \overrightarrow{\mathrm{w}}_{0}} \frac{1}{T} \int_{0}^{T} d t^{\prime}[\hat{n} \times(\hat{n} \times \vec{\beta})] e^{-i \overrightarrow{\mathrm{n}} \cdot \vec{\omega}_{0} t\left(t^{\prime}\right)} .
\end{aligned}
$$

In the limit $r \rightarrow \infty$ 


$$
t\left(t^{\prime}\right)=t^{\prime}+\frac{\left|\overrightarrow{\mathrm{r}}-\vec{\xi}\left(t^{\prime}\right)\right|}{c} \sim t^{\prime}+\frac{r-\overrightarrow{\mathrm{n}} \cdot \vec{\xi}\left(t^{\prime}\right)}{c},
$$

where, for large $r$,

$$
\vec{\eta}_{\overrightarrow{\mathrm{n}}} \sim \lim _{T \rightarrow \infty} i \overrightarrow{\mathrm{n}} \cdot \vec{\omega}_{0} e^{-i \overrightarrow{\mathrm{n}} \cdot \overrightarrow{\mathrm{w}}_{0}} e^{-i \overrightarrow{\mathrm{n}} \cdot \vec{\omega}_{0} r / c} \frac{1}{T} \int_{0}^{T} d t^{\prime}[\hat{n} \times(\hat{n} \times \vec{\beta})] \exp \left[-i \overrightarrow{\mathrm{n}} \cdot \vec{\omega}_{0}\left(t^{\prime}-\frac{\hat{n} \cdot \vec{\xi}\left(t^{\prime}\right)}{c}\right] .\right.
$$

With a similar reasoning for $\mu_{\varphi, \overrightarrow{\mathrm{n}}}$ we have

$$
\vec{\mu}_{\varphi, \overrightarrow{\mathrm{n}}} \sim \lim _{T \rightarrow \infty} i \overrightarrow{\mathrm{n}} \cdot \vec{\omega}_{0} e^{-i \overrightarrow{\mathrm{n}} \cdot \overrightarrow{\mathrm{w}}_{0}} e^{-i \overrightarrow{\mathrm{n}} \cdot \vec{\omega}_{0^{r}} / c} \frac{1}{T} \int_{0}^{T} d t^{\prime}\left\{\hat{n} \times\left[(\hat{n}-\vec{\beta}) \times \vec{\varphi}_{p}\right]\right\} \exp \left\{-i \hat{n} \cdot \vec{\omega}_{0}\left[t^{\prime}-\frac{\hat{n} \cdot \vec{\xi}\left(t^{\prime}\right)}{c}\right]\right\} .
$$

Finally, putting (5.20a) and (5.20b) into (5.18) and taking into account that $\underset{n}{\rightarrow \rightarrow \infty} \hat{r}^{0}$,

$$
\begin{aligned}
G_{1}(\varphi)=\frac{e^{2}}{4 \pi c^{2}}(2 \pi)^{M} \sum_{\overrightarrow{\mathrm{n}}} \int d \Omega\left(\overrightarrow{\mathrm{n}} \cdot \vec{\omega}_{0}\right)^{2} \lim _{T \rightarrow \infty} \lim _{T^{\prime} \rightarrow \infty} \frac{1}{T} \\
\times \int_{0}^{T} d t_{1} \frac{1}{T^{\prime}} \int_{0}^{T^{\prime}} d t_{2}\left\{\hat{r}^{0} \times\left[\left(\hat{r}^{0}-\vec{\beta}\right) \times \vec{\varphi}_{p}\right]\right\}\left(t_{1}\right) \cdot\left[\hat{r}^{0} \times\left(\hat{r}^{0} \times \vec{\beta}\right)\right]\left(t_{2}\right) \\
\quad \times \exp \left[-i \overrightarrow{\mathrm{n}} \cdot \vec{\omega}_{0}\left[\left(t_{1}-t_{2}\right)-\hat{r}^{0} \cdot \frac{\left[\vec{\xi}\left(t_{1}\right)-\vec{\xi}\left(t_{2}\right)\right]}{c}\right)\right] .
\end{aligned}
$$

Now, we relabel $t_{1}-t_{2}=u$ and $t_{1} \rightarrow t$, which, taking into account that

$$
\lim _{T^{\prime} \rightarrow \infty} \frac{1}{T^{\prime}} \int_{0}^{T^{\prime}} d t^{\prime}=\lim _{T^{\prime} \rightarrow \infty} \frac{1}{T^{\prime}} \int_{t-T^{\prime}}^{t} d t^{\prime} \quad \forall t
$$

leads to

$$
\begin{aligned}
& G_{1}(\varphi)=\frac{e^{2}}{4 \pi c^{2}}(2 \pi)^{M} \sum_{\overrightarrow{\mathrm{n}}}\left(\overrightarrow{\mathrm{n}} \cdot \vec{\omega}_{0}\right)^{2} \\
& \quad \times \int d \Omega \lim _{T \rightarrow \infty} \frac{1}{T} \\
& \quad \times \int_{0}^{T} d t \lim _{T^{\prime} \rightarrow \infty} \frac{1}{T^{\prime}} \\
& \quad \times \int_{-T^{\prime}}^{0} d u \times\left\{\hat{r}^{0} \times\left[\left(\hat{r}^{0}-\vec{\beta}\right) \times \vec{\varphi}_{p}\right]\right\}(t) \cdot\left[\hat{r}^{0} \times\left(\hat{r}^{0} \times \vec{\beta}\right)\right](t-u) \\
& \quad \times \exp \left[-i \overrightarrow{\mathrm{n}} \cdot \vec{\omega}_{0}\left[u-\hat{r}^{0} \cdot \frac{[\vec{\xi}(t)-\vec{\xi}(t-u)]}{c}\right]\right] .
\end{aligned}
$$

Finally, expanding the scalar product, taking Eq. (2.9) into account, we have

$$
G_{1}(\varphi)=\frac{e^{2}}{4 \pi c^{2}} \sum_{\overrightarrow{\mathrm{n}}} \int d \overrightarrow{\mathrm{w}}\left(\hat{n}^{\prime} \cdot \vec{\omega}_{0}\right)^{2} \lim _{T \rightarrow \infty} \frac{1}{T} \int_{-T}^{0} d u e^{-\overrightarrow{\mathrm{n}} \cdot \vec{\omega}_{0} u} \sigma[\varphi],
$$

where

$$
\begin{aligned}
\sigma[\varphi]\left(\omega, \overrightarrow{\mathrm{w}}, u ; \vec{\omega}_{0}\right)=\int & d \Omega \exp \left[i \frac{\omega}{c} \hat{r}^{0} \cdot\left(\overrightarrow{\mathrm{r}}-\overrightarrow{\mathrm{r}}^{-u}\right)\right] \\
& \times\left[\vec{\varphi}_{p}\left(I-\hat{r}^{0} \vec{r}^{0}\right) \vec{\beta}^{-u}+\left(\vec{\varphi}_{p} \cdot \hat{r}^{0}\right)\left(\vec{\beta} \cdot \vec{\beta}^{-u}\right)-\left(\vec{\varphi}_{p} \cdot \vec{\beta}^{-u}\right)\left(\vec{\beta} \cdot \hat{r}^{0}\right)\right] .
\end{aligned}
$$


Here, I represents the identity operator, $\hat{r}^{0} \hat{r}^{0}$ a dyadic product, $\overrightarrow{\mathrm{r}}$ means $\overrightarrow{\mathrm{r}}(\overrightarrow{\mathrm{w}})$ (i.e., $\overrightarrow{\mathrm{r}}=\sum_{\overrightarrow{\mathrm{n}}} \vec{\xi}_{\overrightarrow{\mathrm{n}}} e^{i \overrightarrow{\mathrm{n}} \cdot \overrightarrow{\mathrm{w}}}$ ), and, as stated at the end of Sec. II,

$$
\overrightarrow{\mathrm{r}}^{-u}=\sum_{\overrightarrow{\mathrm{n}}} \vec{\xi}_{\overrightarrow{\mathrm{n}}} e^{i\left(\overrightarrow{\mathrm{n}} \cdot \overrightarrow{\mathrm{w}}-\overrightarrow{\mathrm{n}} \cdot \vec{\omega}_{0} u\right)}
$$

and similarly for $\vec{\beta}$.

Equation (5.21) is essentially the expression we are searching for, $G_{1}$. However a more convenient form is obtained if we note that $\sigma$ can be expressed as a Fourier series in the form

$$
\sigma[\varphi]\left(\omega, \overrightarrow{\mathrm{w}}, u ; \vec{\omega}_{0}\right)=\sum_{\overrightarrow{\mathrm{n}}, \overrightarrow{\mathrm{m}}} \sigma_{\overrightarrow{\mathrm{n}}, \overrightarrow{\mathrm{m}}}[\varphi]\left(\omega ; \vec{\omega}_{0}\right) e^{i \overrightarrow{\mathrm{n}} \cdot \overrightarrow{\mathrm{w}}} e^{-i \overrightarrow{\mathrm{m}} \cdot \vec{\omega}_{0} u}
$$

This can be seen by performing the changes

$$
\overrightarrow{\mathrm{w}}-\vec{\omega}_{0} u=\overrightarrow{\mathrm{w}}_{u}
$$

in the expressions of $\overrightarrow{\mathrm{r}}^{-u}$ and $\vec{\beta}^{-u}$. Hence if $\overrightarrow{\mathrm{r}}=\overrightarrow{\mathrm{r}}(\overrightarrow{\mathrm{w}})$, then $\overrightarrow{\mathrm{r}}^{-u}=\overrightarrow{\mathrm{r}}\left(\overrightarrow{\mathrm{w}}_{u}\right)$ and similarly for $\vec{\beta}$. Therefore $\sigma[\varphi]$ depends on $\overrightarrow{\mathrm{w}}$ and $\overrightarrow{\mathrm{w}}_{u}$ through $\overrightarrow{\mathrm{r}}$ and $\vec{\beta}$, and the consequence is a periodic function:

$$
\sigma[\varphi]=\sum_{\overrightarrow{\mathrm{n}}, \overrightarrow{\mathrm{m}}} \bar{\sigma}_{\overrightarrow{\mathrm{n}}, \overrightarrow{\mathrm{m}}}[\varphi] e^{i \overrightarrow{\mathrm{n}} \cdot \overrightarrow{\mathrm{w}}} e^{i \overrightarrow{\mathrm{m}} \cdot \overrightarrow{\mathrm{w}}_{u}}=\sum_{\overrightarrow{\mathrm{n}}, \overrightarrow{\mathrm{m}}} \sigma_{\overrightarrow{\mathrm{n}}, \overrightarrow{\mathrm{m}}}[\varphi] e^{i \overrightarrow{\mathrm{n}} \cdot \overrightarrow{\mathrm{w}}} e^{-i \overrightarrow{\mathrm{m}} \cdot \vec{\omega}_{0^{u}}},
$$

where we have redefined the coefficients and expressed $\overrightarrow{\mathrm{w}}_{u}$ explicitly, which proves (5.23). Putting now that expression in (5.21) and performing the integral

$$
\begin{aligned}
G_{1}(\varphi) & =\frac{e^{2}}{4 \pi c^{2}} \sum_{\overrightarrow{\mathrm{n}}} \int d \overrightarrow{\mathrm{w}}\left(\overrightarrow{\mathrm{n}} \cdot \vec{\omega}_{0}\right)^{2} \lim _{T \rightarrow \infty} \frac{1}{T} \int_{-T}^{0} d u \sum_{\overrightarrow{\mathrm{k}}, \overrightarrow{\mathrm{m}}} \sigma_{\overrightarrow{\mathrm{k}}, \overrightarrow{\mathrm{m}}}[\varphi]\left(\overrightarrow{\mathrm{n}} \cdot \vec{\omega}_{0} ; \vec{\omega}_{0}\right) e^{i \overrightarrow{\mathrm{k}} \cdot \overrightarrow{\mathrm{w}}} e^{-i(\overrightarrow{\mathrm{m}}+\overrightarrow{\mathrm{n}}) \vec{\omega}_{0} u} \\
& =\frac{e^{2}}{4 \pi c^{2}}(2 \pi)^{M} \sum_{\overrightarrow{\mathrm{n}}}\left(\overrightarrow{\mathrm{n}} \cdot \vec{\omega}_{0}\right)^{2} \sigma_{\overrightarrow{0},-\overrightarrow{\mathrm{n}}}[\varphi]\left(\overrightarrow{\mathrm{n}} \cdot \vec{\omega}_{0} ; \vec{\omega}_{0}\right) .
\end{aligned}
$$

On the other hand, taking into account the definition of $\sigma$, we have

$$
\sigma^{*}[\varphi]\left(\omega, \overrightarrow{\mathrm{w}}, u ; \vec{\omega}_{0}\right)=\sigma[\varphi]\left(-\omega, \overrightarrow{\mathrm{w}}, \overrightarrow{\mathrm{u}} ; \vec{\omega}_{0}\right)
$$

so that

$$
\sigma_{-\overrightarrow{\mathrm{n}},-\overrightarrow{\mathrm{m}}}^{*}[\varphi]\left(\omega ; \vec{\omega}_{0}\right)=\sigma_{\overrightarrow{\mathrm{n}}, \overrightarrow{\mathrm{m}}}[\varphi]\left(-\omega ; \vec{\omega}_{0}\right),
$$

and then

$$
\sigma_{\overrightarrow{0},-\overrightarrow{\mathrm{n}}}[\varphi]\left(\overrightarrow{\mathrm{n}} \cdot \vec{\omega}_{0} ; \vec{\omega}_{0}\right)=\sigma_{\overrightarrow{0}, \overrightarrow{\mathrm{n}}}^{*}[\varphi]\left(-\overrightarrow{\mathrm{n}} \cdot \vec{\omega}_{0} ; \vec{\omega}_{0}\right) .
$$

After that (5.24) can be written

$$
\begin{aligned}
G_{2}(\varphi) & =\frac{e^{2}}{4 \pi c^{2}}(2 \pi)^{M} \sum_{\substack{\vec{n} \\
\left(\overrightarrow{\mathrm{n}} \cdot \vec{\omega}_{0}\right)>0}}\left(\overrightarrow{\mathrm{n}} \cdot \vec{\omega}_{0}\right)^{2}\left[\sigma_{\overrightarrow{0},-\overrightarrow{\mathrm{n}}}[\varphi]\left(\overrightarrow{\mathrm{n}} \cdot \vec{\omega}_{0} ; \vec{\omega}_{0}\right)+\sigma_{\overrightarrow{0}, \overrightarrow{\mathrm{n}}}[\varphi]\left(-\overrightarrow{\mathrm{n}} \cdot \vec{\omega}_{0} ; \vec{\omega}_{0}\right)\right] \\
& =\frac{e^{2}}{2 \pi c^{2}}(2 \pi)^{M} \sum_{\overrightarrow{\mathrm{n}}}^{\prime}\left(\overrightarrow{\mathrm{n}} \cdot \omega_{0}\right)^{2} \operatorname{Re}\left[\sigma_{\overrightarrow{0},-\overrightarrow{\mathrm{n}}}[\varphi]\left(\overrightarrow{\mathrm{n}} \cdot \vec{\omega}_{0} ; \vec{\omega}_{0}\right)\right]
\end{aligned}
$$

which is the final expression for the drift coefficient $G_{1}(\varphi)$. Here $\Sigma_{\vec{n}}^{\prime}$ means a sum over those values of $\vec{n}$ that verify $\left(\overrightarrow{\mathbf{n}} \cdot \vec{\omega}_{0}\right)>0$.

\section{Calculation of $\boldsymbol{G}_{2}(\varphi)$}

We start with Eq. (4.2), where the random force $\overrightarrow{\mathrm{F}}^{\text {st }}$ is given by the Lorentz force of the random electromagnetic field 


$$
\overrightarrow{\mathrm{F}}^{\mathrm{st}}=e\left(\overrightarrow{\mathrm{E}}(\overrightarrow{\mathrm{r}}, t)+\frac{\overrightarrow{\mathrm{v}} \times \overrightarrow{\mathrm{B}}(\overrightarrow{\mathrm{r}}, t)}{c}\right) .
$$

A simplication is possible if we take into account the relation (2.11) so that

$$
\frac{\partial J_{j}}{\partial p_{e}^{-u}}=\left.\frac{\partial J_{j}}{\partial p_{e}}\right|_{\substack{\overrightarrow{\vec{p} \rightarrow \overrightarrow{\mathrm{r}}^{-u}} \\ \overrightarrow{\mathrm{p}}-u}}=\left(\frac{\partial x_{e}}{\partial w_{j}}\right)^{-u}
$$

and, besides,

$$
\sum_{j} \omega_{j} \frac{\partial x_{e}}{\partial w_{j}}=\sum_{j} \frac{\partial x_{e}}{\partial w_{j}} \dot{w}_{j}=\dot{x}_{e}=v_{e},
$$

whence it follows

$$
\sum_{i} w_{j} \frac{\partial J_{i}}{\partial p_{e}^{-u}}=v_{e}^{-u}
$$

Then

$$
G_{2}(\varphi)=\mathrm{T}\left[\int_{0}^{\infty} d u \sum_{r, e=1}^{3} \frac{\partial \varphi}{\partial p_{r}} v_{e}^{-u}\left\langle F_{r}^{\mathrm{st}}(\overrightarrow{\mathrm{r}}, \overrightarrow{\mathrm{p}}, 0) F_{e}^{\mathrm{st}}\left(\overrightarrow{\mathrm{r}}^{-u}, \overrightarrow{\mathrm{p}}-u,-u\right)\right\rangle\right] .
$$

The correlations must be calculated from the expression (5.26) of the random force and the field correlations (2.5a) and (2.5b). A straightforward calculation gives

$$
\begin{aligned}
\left\langle F_{r}^{\mathrm{st}}(\overrightarrow{\mathrm{r}}, \overrightarrow{\mathrm{p}}, 0) F_{e}^{\mathrm{st}}\left(\overrightarrow{\mathrm{r}}^{-u}, \overrightarrow{\mathrm{p}}^{-u},-u\right)\right\rangle=\frac{e^{2}}{c^{3}} \int_{0}^{\infty} d \omega^{2} \frac{\omega^{2} \mathscr{Y}^{2}(\omega)}{2} \int d \Omega \cos \left[\frac{\omega}{c} \hat{r}^{0} \cdot\left(\overrightarrow{\mathrm{r}}-\overrightarrow{\mathrm{r}}^{-u}\right)-\omega u\right] & \times\left[\left(\delta_{r e}-r_{r}^{0} r_{e}^{0}\right)+\sum_{i j s}\left(\epsilon_{e i j} \epsilon_{r j s} \beta_{i}^{-u} \hat{r}_{s}^{0}+\epsilon_{r i j} \epsilon_{e j s} \beta_{i} \hat{r}_{s}^{0}\right)\right. \\
& \left.+\sum_{i j \alpha \sigma} \epsilon_{r i j} \epsilon_{e \alpha \sigma} \beta_{i} \beta_{\alpha}^{-u}\left(\delta_{j \sigma}-\hat{r}_{j}^{0} \hat{r}_{\sigma}^{0}\right)\right],
\end{aligned}
$$

where we have changed the notation of $(2.5 \mathrm{a})$ and $(2.5 \mathrm{~b})$ to obtain one similar to the one of $G_{1}(\varphi)$. Hence, and from (5.27), we obtain

$$
\begin{aligned}
& G_{2}(\varphi)=\mathrm{T}\left[\int_{0}^{\infty} d u \int_{0}^{\infty} d \omega \frac{e^{2} \omega^{2} \mathscr{Y}^{2}(\omega)}{2 c^{2}} \int d \Omega \cos \left[\frac{\omega}{c} \hat{r}^{0} \cdot\left(\overrightarrow{\mathrm{r}}-\overrightarrow{\mathrm{r}}^{-u}\right)-\omega u\right]\right. \\
& \left.\times\left[\vec{\varphi}_{p} \cdot\left(I-\hat{r}^{0} \hat{r}^{0}\right) \cdot \vec{\beta}^{-u}+\left(\vec{\varphi}_{p} \cdot \hat{r}^{0}\right)\left(\vec{\beta}^{-} \vec{\beta}^{-u}\right)-\left(\vec{\varphi}_{p} \cdot \vec{\beta}^{-u}\right)\left(\vec{\beta} \cdot \hat{r}^{0}\right)\right]\right) .
\end{aligned}
$$

Now, taking Eq. (5.22) into account, we have

$$
G_{2}(\varphi)=\frac{e^{2}}{4 c^{2}} \int d \overrightarrow{\mathrm{w}} \int_{0}^{\infty} d u \int_{0}^{\infty} d \omega \omega^{2} \mathscr{Y}^{2}(\omega)\left[e^{-i \omega u} \sigma[\varphi]\left(\omega, \overrightarrow{\mathrm{w}}, u ; \vec{\omega}_{0}\right)+e^{i \omega u} \sigma[\varphi]\left(-\omega, \overrightarrow{\mathrm{w}}, u ; \vec{\omega}_{0}\right)\right]
$$

Inserting here the Fourier expansion of $\sigma[\varphi]$ we have terms of the form

$$
\int_{0}^{\infty} d u e^{-i\left(\omega+\overrightarrow{\mathrm{m}} \cdot \overrightarrow{\mathrm{w}}_{0}\right) u}=\pi \delta\left(\omega+\overrightarrow{\mathrm{m}} \cdot \vec{\omega}_{0}\right)-i V_{P} \frac{1}{\omega+\overrightarrow{\mathrm{m}} \cdot \vec{\omega}_{0}} .
$$

It is clear that the second term will not appear if the integral were from $-\infty$ to $+\infty$. It can be seen that 
this is only possible if $G^{\mu \nu}=G^{\nu \mu}$, which is not true in general. However, it is convenient to avoid that term, which will be possible because, as we shall prove, it does not contribute to the Fokker-Planck equation. In the following we shall call $G_{2}^{s}$ (symmetrized) an expression like $G_{2}$ but with the integral in $u$ going from $-\infty$ to $+\infty$ (with a factor $\frac{1}{2}$ inserted), i.e.,

$$
G_{2}^{s}(\varphi)=\frac{e^{2}}{8 c^{2}} \int d \overrightarrow{\mathrm{w}} \int_{-\infty}^{+\infty} d u \int_{0}^{\infty} d \omega \omega^{2} \mathscr{Y}^{2}(w)\left[e^{-i \omega u} \sigma[\varphi]\left(\omega, \overrightarrow{\mathrm{w}}, u ; \vec{\omega}_{0}\right)+e^{i \omega u} \sigma[\varphi]\left(-\omega, \overrightarrow{\mathrm{w}}, u ; \vec{\omega}_{0}\right)\right]
$$

\section{Relation between $G_{2}^{s}$ and $G_{1}$}

Before studying the possibility of replacing $G_{2}^{s}$ instead of $G_{2}$ we show that $G_{2}^{s}$ has a rather simple relation with $G_{1}$.

Putting (5.23) into (5.30) and evaluating the integral we have

$$
\begin{aligned}
& G_{2}^{s}=\frac{e^{2}}{8 c^{2}} \int d \overrightarrow{\mathrm{w}} \int_{-\infty}^{+\infty} d u \int_{0}^{\infty} d \omega \omega^{2} \mathscr{Y}^{2}(\omega) \sum_{\overrightarrow{\mathrm{n}}, \overrightarrow{\mathrm{m}}}\left[e^{-i \omega u} \sigma_{\overrightarrow{\mathrm{n}}, \overrightarrow{\mathrm{m}}}[\varphi]\left(\omega ; \vec{\omega}_{0}\right) e^{i \overrightarrow{\mathrm{n}} \cdot \overrightarrow{\mathrm{w}}} e^{-i \overrightarrow{\mathrm{m}} \cdot \vec{\omega}_{0} u}\right. \\
& \left.+e^{i \omega u} \sigma_{\overrightarrow{\mathrm{n}}, \overrightarrow{\mathrm{m}}}[\varphi]\left(-\omega ; \vec{\omega}_{0}\right) e^{i \overrightarrow{\mathrm{n}} \cdot \overrightarrow{\mathrm{w}}} e^{-i \overrightarrow{\mathrm{m}} \cdot \vec{\omega}_{0} u}\right] \\
& =\frac{e^{2}}{8 c^{2}}(2 \pi)^{M} \int_{0}^{\infty} d \omega \omega^{2} \mathscr{Y}^{2}(\omega) \sum_{\overrightarrow{\mathrm{m}}}(2 \pi)\left[\delta\left(\omega+\overrightarrow{\mathrm{m}} \cdot \vec{\omega}_{0}\right) \sigma_{\overrightarrow{0}, \overrightarrow{\mathrm{m}}}[\varphi]\left(\omega ; \vec{\omega}_{0}\right)\right. \\
& \left.+\delta\left(\omega-\overrightarrow{\mathrm{m}} \cdot \vec{\omega}_{0}\right) \sigma_{\overrightarrow{0}, \overrightarrow{\mathrm{m}}}[\varphi]\left(-\omega ; \vec{\omega}_{0}\right)\right] \\
& =\frac{e^{2}}{4 c^{2}}(2 \pi)^{M}\left[\sum_{\substack{\overrightarrow{\mathrm{m}} \\
\overrightarrow{\mathrm{m}} \cdot \vec{\omega}_{0}<0}}\left(\overrightarrow{\mathrm{m}} \cdot \vec{\omega}_{0}\right)^{2} \mathscr{Y}^{2}\left(\overrightarrow{\mathrm{m}} \cdot \vec{\omega}_{0}\right) \sigma_{\overrightarrow{0}, \overrightarrow{\mathrm{m}}}[\varphi]\left(-\overrightarrow{\mathrm{m}} \cdot \vec{\omega}_{0} ; \vec{\omega}_{0}\right)\right.
\end{aligned}
$$

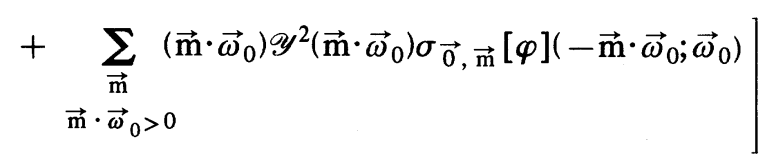

$$
\begin{aligned}
& =\frac{e^{2} \pi}{2 c^{2}}(2 \pi)^{M} \sum_{\overrightarrow{\mathrm{n}}}^{\prime}\left(\overrightarrow{\mathrm{n}} \cdot \vec{\omega}_{0}\right)^{2} \mathscr{Y}^{2}\left(\overrightarrow{\mathrm{n}} \cdot \vec{\omega}_{0}\right) \operatorname{Re}\left[\sigma_{\overrightarrow{0}, \overrightarrow{\mathrm{n}}}[\varphi]\left(\overrightarrow{\mathrm{n}} \cdot \vec{\omega}_{0} ; \vec{\omega}_{0}\right)\right]
\end{aligned}
$$

Now, the Rayleigh-Jeans spectrum is $u(\omega)=$ const $\times \omega^{2}$ so that, taking (2.6) into account, $\mathscr{Y}$ is a constant. Then, a comparison between (5.31) and (5.25) gives

$$
G_{2}^{s}(\varphi)=\pi^{2} \mathscr{Y}^{2} G_{1}(\varphi)
$$

which is the desired relation.

Now we must consider the relation between $G_{2}$ and $\boldsymbol{G}_{2}^{\boldsymbol{s}}$.

\section{E. Symmetrization of $\boldsymbol{G}_{2}$}

It is unfortunate that in the analysis of the possibility of symmetrizing $G_{2}$ it is unavoidable that the explicit calculation of the integral (5.28) is rather lengthy. Also, our result will be only valid for a Rayleigh-Jeans spectrum. Although this is all we need in the present paper, it would be desirable to have a result independent of the spectrum. In the case of a central potential independent of the velocity, it is proved in Appendix D that, indeed, $G_{2}=G_{2}^{s}$ for any spectrum. Also, in the case that the random force does not depend on the physical variables, if the system is multiperiodic, and nondegenerate, the diffusion matrix is symmetrical. ${ }^{19}$ However, this is not proved in the general case, and we must restrict ourselves to the Rayleigh-Jeans spectrum. 
This spectrum present the difficulty that it gives rise to divergent integrals and it is necessary to introduce a cutoff, which may be removed at the end. We shall use a cutoff Rayleigh-Jeans spectrum in the form

$$
S(\omega)=\text { const } \times \omega^{2} e^{-\epsilon \omega} \quad(\epsilon>0) .
$$

This fact can be related with the structure of the charge. Note that in the Lorentz-Dirac model we are using, it is supposed that particles are points. We think that if an extended particle is considered, the effective spectrum will change considerably at high frequencies. This will be discussed in the second part of this work.

Introducing (5.33) in Eq. (5.28) we perform the solid-angle integral, whose details are given in Appendix B. We obtain

$$
\begin{array}{r}
G_{2}(\varphi)=\frac{e^{2} \mathscr{Y}^{2}}{2 c^{2}} 4 \pi \int d \overrightarrow{\mathrm{w}} \int_{0}^{\infty} d \omega\left[I_{1}(\epsilon)+I_{2}(\epsilon)\right. \\
\left.+I_{3}(\epsilon)\right]
\end{array}
$$

where

$$
\begin{aligned}
& I_{1}(\epsilon)=P[\varphi](u) \int_{0}^{\infty} d \omega \omega^{2} e^{-\epsilon \omega} \cos \omega u \frac{\sin \omega \alpha}{\omega \alpha}, \\
& \begin{aligned}
& I_{2}(\epsilon)=Q[\varphi](u) \int_{0}^{\infty} d \omega \omega^{2} e^{-\epsilon \omega} \\
& \times \cos \omega u\left(\frac{\cos \omega \alpha}{\omega^{2} \alpha^{2}}-\frac{\sin \omega \alpha}{\omega^{3} \alpha^{3}}\right),
\end{aligned}
\end{aligned}
$$

$$
\begin{aligned}
I_{3}(\epsilon)=R[\varphi](u) \int_{0}^{\infty} & d \omega \omega^{2} e^{-\epsilon \omega} \\
& \times \sin \omega u\left(\frac{\sin \omega \alpha}{\omega^{2} \alpha^{2}}-\frac{\cos \omega \alpha}{\omega \alpha}\right),
\end{aligned}
$$

$P[\varphi]=\vec{\varphi}_{p} \cdot \vec{\beta}^{-u}-\left(\vec{\varphi}_{p} \cdot \hat{n}_{u}\right)\left(\vec{\beta}^{-u} ; \hat{n}_{u}\right)$,

$Q[\varphi]=\vec{\varphi}_{p} \cdot \vec{\beta}^{-u}-3\left(\vec{\varphi}_{p} \cdot \hat{n}_{u}\right)\left(\vec{\beta}^{-u} \cdot \hat{n}_{u}\right)$,

$R[\varphi]=\left(\hat{n}_{u} \cdot \vec{\varphi}_{p}\right)\left(\vec{\beta} \cdot \vec{\beta}^{-u}\right)-\left(\widehat{n}_{u} \cdot \vec{\beta}\right)\left(\vec{\varphi}_{p} \cdot \vec{\beta}^{-u}\right)$, and

$$
\alpha=\frac{\left|\overrightarrow{\mathrm{r}}-\overrightarrow{\mathrm{r}}^{-u}\right|}{c}, \quad \hat{n}_{u}=\frac{\overrightarrow{\mathrm{r}}-\overrightarrow{\mathrm{r}}^{-u}}{\left|\overrightarrow{\mathrm{r}}-\overrightarrow{\mathrm{r}}^{-u}\right|} .
$$

The integrals are only needed to lowest order in $\epsilon$ which gives, after a tedious calculation summarized in Appendix C,

$$
\int_{0}^{\infty} d u I_{1}(\epsilon)=\frac{1}{\epsilon} T_{1}[\varphi]+\frac{1}{2} \int_{-\infty}^{+\infty} d u I_{1}(0)+r_{\epsilon}^{\prime},
$$

$\int_{0}^{\infty} d u I_{2}(\epsilon)=\frac{1}{\epsilon} T_{2}[\varphi]+\frac{1}{2} \int_{-\infty}^{+\infty} d u I_{2}(0)+r_{\epsilon}^{\prime \prime}$,

$$
\int_{0}^{\infty} d u I_{3}(\epsilon)=\frac{1}{\epsilon} T_{3}[\varphi]+\frac{1}{2} \int_{-\infty}^{+\infty} d u I_{3}(0)+r_{\epsilon}^{\prime \prime \prime},
$$

where $r_{\epsilon}^{\prime}, r_{\epsilon}^{\prime \prime}$, and $r_{3}^{\prime \prime \prime}$ go to zero with $\epsilon$,

$$
T=T_{1}+T_{2}+T_{3}=d_{1}(\beta) \Omega_{1}[\varphi]+d_{2}(\beta) \Omega_{2}[\varphi]
$$

and

$$
\begin{aligned}
& \Omega_{1}[\varphi]=\vec{\varphi}_{p} \cdot \dot{\vec{\beta}}, \\
& \Omega_{2}[\varphi]=\frac{\left(\vec{\varphi}_{p} \cdot \vec{\beta}\right)(\vec{\beta} \cdot \dot{\vec{\beta}})}{\beta^{2}}, \\
& d_{1}(\beta)=\frac{1}{2}\left(1+\frac{1}{\beta^{2}}\right)\left(\frac{1}{2 \beta} \ln \frac{1+\beta}{1-\beta}\right)-\frac{1}{2 \beta^{2}},
\end{aligned}
$$

$$
d_{2}(\beta)=-\frac{1}{2}\left(1+\frac{3}{\beta^{2}}\right)\left(\frac{1}{2 \beta} \ln \frac{1+\beta}{1-\beta}\right)+\frac{3}{2 \beta^{2}}+\gamma^{2} .
$$

Finally, we have

$$
\begin{aligned}
G_{2}(\varphi)= & G_{2}^{s}(\varphi)+\frac{e^{2} \mathscr{Y}^{2} 2 \pi}{c^{2}} \frac{1}{\epsilon} \\
& \times \int d \overrightarrow{\mathrm{w}} T[\varphi]+r_{\epsilon},
\end{aligned}
$$

which is the relation searched. 


\section{SOLUTION OF THE FOKKER-PLANCK EQUATION}

From the expressions (5.32) and (5.42) for the coefficients, we have

$$
\begin{aligned}
0= & \sum_{i=1}^{M} \frac{\partial}{\partial J_{i}}\left[G_{1}\left(J_{i}\right) W_{0}+\pi^{2} \mathscr{Y}^{2} G_{1}\left(J_{i}\right) W_{0}^{\prime}+\frac{e^{2} \mathscr{Y}^{2}}{c^{2}} 2 \pi \frac{1}{\epsilon} \int d \overrightarrow{\mathrm{w}} T\left[J_{i}\right] W_{0}^{\prime}\right] \\
& +\sum_{i=M+1}^{3} \frac{\partial}{\partial J_{i}^{\prime}}\left[G_{1}\left(J_{i}^{\prime}\right) W_{0}+\pi^{2} \mathscr{Y}^{2} G_{1}\left(J_{i}^{\prime}\right) W_{0}^{\prime}+\frac{e^{2} \mathscr{Y}^{2} 2 \pi}{c^{2}} \frac{1}{\epsilon} \int d \overrightarrow{\mathrm{w}} T\left[J_{i}^{\prime}\right] W_{0}^{\prime}\right] \\
& +\sum_{i=M+1}^{3} \frac{\partial}{\partial w_{i}^{\prime}}\left[G_{1}\left(w_{i}^{\prime}\right) W_{0}+\pi^{2} \mathscr{Y}^{2} G_{1}\left(w_{i}^{\prime}\right) W_{0}^{\prime}+\frac{e^{2} \mathscr{Y}^{2} 2 \pi}{c^{2}} \frac{1}{\epsilon} \int d \overrightarrow{\mathrm{w}} T\left[w_{i}^{\prime}\right] W_{0}^{\prime}\right]+r_{\epsilon}
\end{aligned}
$$

In the first place we shall prove that terms of order $1 / \epsilon$ do not contribute, i.e., that

$$
\sum_{i=1}^{M} \frac{\partial}{\partial J_{i}}\left(W_{0}^{\prime} \int d \overrightarrow{\mathrm{w}} T\left[J_{i}\right]\right)+\sum_{i=M+1}^{3}\left[\frac{\partial}{\partial J_{i}^{\prime}}\left(W_{0}^{\prime} \int d \overrightarrow{\mathrm{w}} T\left[J_{i}^{\prime}\right]\right)+\frac{\partial}{\partial w_{i}^{\prime}}\left(W_{0}^{\prime} \int d \overrightarrow{\mathrm{w}} T\left[w_{i}^{\prime}\right]\right]\right]=0 .
$$

As the energy depends on $J_{1}, \ldots, J_{M}$ and $W_{0}^{\prime}$ is a function of the energy, it is clear that

$$
\frac{\partial W_{0}^{\prime}}{\partial J_{i}^{\prime}}=\frac{\partial W_{0}^{\prime}}{\partial w_{i}^{\prime}} \equiv 0,
$$

so that the previous relation can be written

$$
\sum_{i=1}^{M} \frac{\partial}{\partial J_{i}}\left(W_{0}^{\prime} \int d \overrightarrow{\mathrm{w}} T\left[J_{i}\right]\right)+\sum_{i=M+1}^{3} W_{0}^{\prime}\left\{\frac{\partial}{\partial J_{i}^{\prime}} \int d \overrightarrow{\mathrm{w}} T\left[J_{i}^{\prime}\right]+\frac{\partial}{\partial w_{i}^{\prime}} \int d \overrightarrow{\mathrm{w}} T\left[w_{i}^{\prime}\right]\right\}=0 .
$$

We shall prove that the first and the second terms are zero independently. We start by deriving the following relation:

$$
\int d \overrightarrow{\mathrm{w}} T[\varphi]=-\int d \overrightarrow{\mathrm{w}}\left(\dot{\vec{\varphi}}_{p} \cdot \vec{\beta}\right) d_{1}(\beta) .
$$

To do that, let us write

$$
\begin{aligned}
\frac{d}{d t}\left[d_{1}(\beta)\left(\vec{\varphi}_{p} \cdot \vec{\beta}\right)\right]= & \left(\dot{\vec{\varphi}}_{p} \cdot \vec{\beta}\right) d_{1}(\beta)+\left(\vec{\varphi}_{p} \cdot \dot{\vec{\beta}}\right) d_{1}(\beta) \\
& +\left(\vec{\varphi}_{p} \cdot \vec{\beta}\right) \frac{d}{d t} d_{1}(\beta)
\end{aligned}
$$

Now we prove that the last two terms together give $T[\varphi]$. To do that we define

$$
\bar{d}_{i}(y) \equiv d_{i}(\sqrt{y}), \quad i=1,2
$$

which is possible because $d_{i}$ are even functions of their arguments [see Eqs. (5.41a) and (5.41b)]. These functions fulfill the equality

$$
\frac{d}{d y} \bar{d}_{1}(y)=\frac{1}{2 y} \bar{d}_{2}(y) .
$$

Therefore, as

$$
\begin{aligned}
\frac{d}{d t} d_{1}(\beta) & =\frac{d}{d t} \bar{d}_{1},\left(\beta^{2}\right) \\
& =2 \vec{\beta} \cdot \dot{\vec{\beta}} \frac{\bar{d}_{2}\left(\beta^{2}\right)}{2 \beta^{2}} \\
& =\frac{\vec{\beta} \cdot \dot{\vec{\beta}}}{\beta^{2}} d_{2}(\beta),
\end{aligned}
$$

taking (5.40a) and (5.40b) and (5.39) into account, we see that

$$
\frac{d}{d t}\left[d_{1}(\beta)\left(\vec{\varphi}_{p} \cdot \vec{\beta}\right)\right]=\left(\dot{\vec{\varphi}}_{p} \cdot \beta\right) d_{1}(\beta)+T[\varphi]
$$

Now, using again (2.9)

$$
\begin{aligned}
\int d \overrightarrow{\mathrm{w}} \frac{d}{d t}\left[d_{1}(\beta)\left(\vec{\varphi}_{p} \cdot \vec{\beta}\right)\right] & \\
& =\lim _{T \rightarrow \infty} \frac{1}{T} \int_{0}^{T} d t \frac{d}{d t}\left[d_{1}(\beta)\left(\vec{\varphi}_{p} \cdot \beta\right)\right] \\
& =\left.\lim _{T \rightarrow \infty} \frac{1}{T} d_{1}(\beta)\left(\vec{\varphi}_{p} \cdot \vec{\beta}\right)\right|_{0} ^{T} .
\end{aligned}
$$


defined functions at the origin and take values for $\beta \in[0,1)$. On the other hand, the values of the constants $J_{i}, J_{i}^{\prime}$, and $w_{i}^{\prime}$ put bounds to the possible values of $\beta$ so that $d_{1}$ and $d_{2}$ are bounded functions. Finally, $\vec{\varphi}_{p}$ is a periodic function of the variables $\overrightarrow{\mathrm{w}}$. Its value at time $T$ will be one of the values they take in the closed interval $[0,2 \pi]^{M}$. This being compact and $\vec{\varphi}_{p}$ a continuous function, it is bounded. Therefore, in the limit $T \rightarrow \infty, \mathrm{Eq}$. (6.6) goes to zero, so that from (6.5), it follows (6.3).

Let us now prove that the first term of (6.2) is zero. For this term $\varphi=J_{i}$ and $\vec{\varphi}_{p}=\vec{\nabla}_{p} J_{i}$. Using the relations $(2.11)$,

$$
\vec{\varphi}_{p}=\frac{\partial \overrightarrow{\mathbf{r}}}{\partial w_{i}}
$$

If we put

$$
\overrightarrow{\mathrm{r}}=\sum_{\overrightarrow{\mathrm{n}}} \vec{\xi}_{\overrightarrow{\mathrm{n}}} e^{i \overrightarrow{\mathrm{n}} \cdot \overrightarrow{\mathrm{w}}} \Longrightarrow \vec{\varphi}_{p}=\sum_{n} \vec{\xi}_{\overrightarrow{\mathrm{n}}} i n_{i} e^{i \overrightarrow{\mathrm{n}} \cdot \overrightarrow{\mathrm{w}}}
$$

and

$$
\dot{\vec{\varphi}}_{p}=\sum_{\overrightarrow{\mathrm{n}}} \xi_{\overrightarrow{\mathrm{n}}} i \overrightarrow{\mathrm{n}} \cdot \vec{\omega}_{0} i \overrightarrow{\mathrm{n}}_{i} e^{i \overrightarrow{\mathrm{n}} \cdot \overrightarrow{\mathrm{w}}}=c \frac{\partial \vec{\beta}}{\partial w_{i}},
$$

because $\vec{\xi}_{\vec{n}}$ depends only on $J_{i}, J_{i}^{\prime}$, and $w_{i}^{\prime}$ whereas $\vec{\omega}_{0}$ depends only on $\vec{J}$.

On the other hand, the function $\bar{d}_{1}(y)$ is continuous and, therefore integrable. Let us define $\bar{d}_{3}(y)$ such that

$$
\frac{d}{d y} \bar{d}_{3}(y)=\bar{d}_{1}(y)
$$

and

$$
d_{3}(\beta)=\bar{d}_{3}\left(\beta^{2}\right),
$$

which gives

$$
\begin{aligned}
\left(\dot{\vec{\varphi}}_{p} \cdot \vec{\beta}\right) d_{1}(\beta) & =c \frac{\partial \vec{\beta}}{\partial w_{i}} \cdot \vec{\beta} d_{1}(\beta) \\
& =\frac{c}{2} \frac{\partial}{\partial w_{i}} d_{3}(\beta) .
\end{aligned}
$$

Then, Eq. (6.3) gives

$$
\begin{aligned}
\int d \overrightarrow{\mathrm{w}} T\left[J_{i}\right] & =-\int d \overrightarrow{\mathrm{w}} \frac{c}{2} \frac{\partial}{\partial w_{i}} d_{3}(\beta) \\
& =-\int \prod_{r \neq i} d w_{r} \int_{0}^{2 \pi} d w_{i} \frac{\partial}{\partial w_{i}} d_{3}(\beta) \\
& =0
\end{aligned}
$$

because $d_{3}(\beta)$ is a periodic function of $w_{i}$.

Finally, we prove that the second term of (6.2) is also zero. If $\varphi=J_{i}^{\prime}$,

$$
\vec{\varphi}_{p}=-\frac{\partial \overrightarrow{\mathrm{r}}}{\partial w_{i}^{\prime}}=\sum_{\overrightarrow{\mathrm{n}}} \frac{\partial \vec{\xi}_{\overrightarrow{\mathrm{n}}}}{\partial w_{i}^{\prime}} e^{i \overrightarrow{\mathrm{n}} \cdot \overrightarrow{\mathrm{w}}}
$$

then

$$
\dot{\vec{\varphi}}_{p}=\sum_{\overrightarrow{\mathrm{n}}} \frac{\partial \vec{\xi}_{\overrightarrow{\mathrm{n}}}}{\partial w_{i}^{\prime}} i \overrightarrow{\mathrm{n}} \cdot \vec{\omega}_{0} e^{i \overrightarrow{\mathrm{n}} \cdot \overrightarrow{\mathrm{w}}}=c \frac{\partial \vec{\beta}}{\partial w_{i}^{\prime}}
$$

for the same reasons as before. Then, we have

$$
\begin{aligned}
\frac{\partial}{\partial J_{i}^{\prime}} \int d \overrightarrow{\mathrm{w}} T\left[J_{i}^{\prime}\right] & =\frac{\partial}{\partial J_{i}^{\prime}}\left[-\int d \overrightarrow{\mathrm{w}}\left(\dot{\vec{\varphi}}_{p} \cdot \vec{\beta}\right) d_{1}(\beta)\right] \\
& =\frac{\partial}{\partial J_{i}^{\prime}}\left[-\int d \overrightarrow{\mathrm{w}} \frac{c}{2} \frac{\partial d_{3}(\beta)}{\partial w_{i}^{\prime}}\right] \\
& =-\frac{c}{2} \frac{\partial^{2}}{\partial J_{i}^{\prime} \partial w_{i}^{\prime}} \int d \overrightarrow{\mathrm{w}} d_{3}(\beta)
\end{aligned}
$$

where we have used (6.3). For $\varphi=w_{i}^{\prime}$,

$$
\begin{aligned}
\vec{\varphi}_{p} & =\vec{\nabla}_{p} w_{i}^{\prime}=-\frac{\partial \overrightarrow{\mathrm{r}}}{\partial J_{i}^{\prime}} \\
& =-\sum_{n} \frac{\partial \vec{\xi}_{\overrightarrow{\mathrm{n}}}}{\partial J_{i}^{\prime}} e^{i \overrightarrow{\mathrm{n}} \cdot \overrightarrow{\mathrm{w}}}, \\
\dot{\vec{\varphi}}_{p} & =-\sum_{\overrightarrow{\mathrm{n}}} \frac{\partial \vec{\xi}_{\overrightarrow{\mathrm{n}}}}{\partial J_{i}^{\prime}} i \overrightarrow{\mathrm{n}} \cdot \vec{\omega}_{0} e^{i \overrightarrow{\mathrm{n}} \cdot \overrightarrow{\mathrm{w}}} \\
& =-c \frac{\partial \vec{\beta}}{\partial J_{i}^{\prime}} .
\end{aligned}
$$

Then, in this case

$$
\begin{aligned}
\left.\frac{\partial}{\partial w_{i}^{\prime}} \iint d \overrightarrow{\mathrm{w}} T\left[w_{i}^{\prime}\right]\right) & \\
& =\frac{\partial}{\partial w_{i}^{\prime}}\left[-\int d \overrightarrow{\mathrm{w}}\left(\dot{\vec{\varphi}}_{p} \cdot \vec{\beta}\right) d_{1}(\vec{\beta})\right] \\
& =\frac{\partial}{\partial w_{i}^{\prime}}\left[-\int d \overrightarrow{\mathrm{w}}\left[-\frac{c}{2}\right] \frac{\partial}{\partial J_{i}^{\prime}} d_{3}(\beta)\right] \\
& =\frac{c}{2} \frac{\partial^{2}}{\partial w_{i}^{\prime} \partial J_{i}^{\prime}} \int d \overrightarrow{\mathrm{w}} d_{3}(\beta) .
\end{aligned}
$$

Hence, adding (6.8) and (6.9), we obtain the relation

$$
\frac{\partial}{\partial J_{i}^{\prime}} \int d \overrightarrow{\mathrm{w}} T\left[J_{i}^{\prime}\right]+\frac{\partial}{\partial w_{i}^{\prime}} \int d \overrightarrow{\mathrm{w}} T\left[w_{i}^{\prime}\right] \equiv 0 .
$$

From this equation, and (6.7) it follows Eq. (6.2).

Once we have shown that all terms of order $1 / \epsilon$ in the Fokker-Planck equation (6.7) cancel out, we may take the limit $\epsilon \rightarrow 0$, which gives 


$$
\begin{aligned}
0=\sum_{i=1}^{M} \frac{\partial}{\partial J_{i}}\left[G_{1}\left(J_{i}\right)\left(W_{0}+\pi^{2} \mathscr{Y}^{2} W_{0}^{\prime}\right)\right]+\sum_{i=M+1}^{3} & \left(\frac{\partial}{\partial J_{i}^{\prime}}\left[G_{1}\left(J_{i}^{\prime}\right)\left(W_{0}+\pi^{2} \mathscr{Y}^{2} W_{0}^{\prime}\right)\right]\right. \\
& \left.+\frac{\partial}{\partial w_{i}^{\prime}}\left[G_{1}\left(w_{i}^{\prime}\right)\left(W_{0}+\pi^{2} \mathscr{Y}^{2} W_{0}^{\prime}\right)\right]\right] .
\end{aligned}
$$

It is easy to realize that the function

$$
W_{0}=\text { const } \times \exp \left(-\frac{\mathscr{C}}{\pi^{2} \mathscr{Y}^{2}}\right)
$$

is a solution of that equation. As the potential $V$ goes to $\infty$ quickly enough in the limit $r \rightarrow \infty$, it is clear that this solution is normalizable and, therefore, unique, which is the result we desired.

\section{CONCLUSIONS}

We have shown that the stationary state of a relativistic multiply periodic system in the presence of an electromagnetic radiation with RayleighJeans spectrum is given by the Maxwell-Boltzmann distribution. This definitely contradicts the Boyer claim that Maxwell-Boltzmann distribution and Rayleigh-Jeans spectrum are incompatible in relativistic theory. ${ }^{3}$

As an interesting byproduct, we have proven that in the Fokker-Planck equation, written in the form of the divergence of a current, the damping and the stochastic force appear separately. In fact, the damping gives rise to the drift and the stochastic force to the diffusion. Also, the diffusion matrix is symmetric, at least for the Rayleigh-Jeans spectrum and for any spectrum in the case of a central potential. The separation between drift and diffusion is due to the fact that the drift term is related to the dissipation of energy by the particle (this can be seen in V A) and the diffusion term is related to the increase in energy of the particle due to the background field, which will be seen more clearly in the second part of the paper.

Finally, we must point out that if the system is on the equilibrium state corresponding to the solution of the stationary Fokker-Planck equation (in our case, the relativistic Maxwell-Boltzmann distribution), this fact only guarantees that there is global energetic equilibrium with the radiation. Nevertheless, the equilibrium should exist at each frequency (radiative equilibrium, related to Kirchhoff's law). If this is not fulfilled, the radia- tion is not in equilibrium, but its spectrum is continuously changing. The study of this condition, which is essential for the foundations of classical relativistic statistical mechanics and the study of the blackbody spectrum, will be made in the second part of the paper. We shall show that there exists radiative equilibrium between a radiation with Rayleigh-Jeans spectrum and a relativistic multiply periodic material system.

\section{APPENDIX A}

We shall prove the relation (5.13). Let $\rho=1-\vec{\beta} \cdot \hat{r}^{0}$. An easy calculation gives

$$
\begin{aligned}
\overrightarrow{\mathrm{g}}^{\infty} & =\frac{1}{\rho^{3}}\left(\hat{r}^{0} \cdot \dot{\vec{\beta}}\right)\left(\hat{r}_{0}-\vec{\beta}\right)-\frac{1}{\rho^{2}} \dot{\vec{\beta}}, \\
\overrightarrow{\mathrm{h}}_{\varphi}^{\infty} & =\frac{1}{\rho} \frac{d}{d t}\left[\frac{\left(\hat{r}^{0} \cdot \vec{\varphi}_{p}\right)\left(\hat{r}^{0}-\vec{\beta}\right)-\rho \vec{\varphi}_{p}}{\rho}\right] \\
& =\overrightarrow{\mathrm{h}}^{(1)}+\overrightarrow{\mathrm{h}}^{(2)},
\end{aligned}
$$

where

$$
\begin{aligned}
& \overrightarrow{\mathrm{h}}^{(1)}=\frac{1}{\rho}\left[-\dot{\vec{\varphi}}_{p}+\frac{\left(\hat{r}_{0}-\vec{\beta}\right)}{\rho}\left(\hat{r}_{0} \cdot \dot{\vec{\varphi}}_{p}\right)\right], \\
& \overrightarrow{\mathrm{h}}^{(2)}=\frac{1}{\rho}\left(\hat{r}_{0} \cdot \vec{\varphi}_{p}\right) \frac{d}{d t}\left[\frac{\hat{r}_{0}-\vec{\beta}}{\rho}\right] .
\end{aligned}
$$

Writing $I=I_{1}+I_{2}$ with $I_{i}=\int d \Omega \overrightarrow{\mathrm{h}}^{(i)} \cdot \overrightarrow{\mathrm{g}}^{\infty} \rho$, we calculate separately each integral. From (A1) and (A3a) we have

$$
I_{1}=\int d \Omega \rho\left[\frac{1}{\rho^{3}}\left(\hat{r}^{0} \cdot \dot{\vec{\beta}}\right)\left(\hat{r}^{0}-\vec{\beta}\right)-\frac{1}{\rho^{2}} \dot{\vec{\beta}}\right] \cdot\left[\frac{-\dot{\vec{\varphi}}_{p}+\left[\left(\hat{r}^{0}-\vec{\beta}\right) / \rho\right]\left(\hat{r}^{0} \cdot \dot{\vec{\varphi}}_{p}\right)}{\rho}\right],
$$

which, after a straightforward calculation, can be written as 


$$
I_{1}=\int d \Omega \frac{1}{\rho^{4}}\left\{\left(\hat{r}^{0} \cdot \dot{\vec{\varphi}}_{p}\right)\left(\overrightarrow{\mathrm{r}}^{0} \cdot \dot{\vec{\beta}}\right)\left(\beta^{2}-1\right)+\rho\left[(\vec{\beta} \cdot \dot{\vec{\beta}})\left(\hat{r}^{0} \cdot \dot{\vec{\varphi}}_{p}\right)+\left(\vec{\beta} \cdot \dot{\vec{\varphi}}_{p}\right)\left(\hat{r}^{0} \cdot \dot{\vec{\beta}}\right)\right]+\rho^{2}(\dot{\vec{\beta}} \cdot \dot{\vec{\varphi}} p)\right\}
$$

choosing the coordinate axes such that $0 Z$ is in the direction of $\vec{\beta}$, it turns out that $\rho=1-\beta \cos \theta$ does not depend on $\varphi$ and also $\beta_{x}=\beta_{y}=0$. Moreover, for any vector $\overrightarrow{\mathrm{A}}$,

$$
A_{z}=\frac{\overrightarrow{\mathrm{A}} \cdot \vec{\beta}}{\beta} \text {. }
$$

Concerning the integration over the azimuthal angle $\varphi$, three kinds of terms appear in (A5):
(a) $\int d \Omega g(\theta)=2 \pi \int_{0}^{\pi} \sin \theta g(\theta) d \theta$,
(b) $\int d \Omega g(\theta)\left(\hat{r}^{0} \cdot \vec{a}\right)=2 \pi a_{z} \int_{0}^{\pi} \sin \theta \cos \theta g(\theta) d \theta$,
(c) $\int d \Omega g(\theta)\left(\hat{r}_{0} \cdot \overrightarrow{\mathrm{a}}\right)\left(\hat{r}_{0} \cdot \overrightarrow{\mathrm{b}}\right)=2 \pi \int_{0}^{\pi} d \theta g(\theta) \sin \theta\left[\frac{1}{2}\left(a_{x} b_{x}+a_{y} b_{y}\right) \sin ^{2} \theta+a_{z} b_{z} \cos ^{2} \theta\right]$.

After a few simple calculations and taking (A6) and (A7) into account, (A5) becomes

$$
\begin{aligned}
I_{1}= & 2 \pi\left(\dot{\vec{\varphi}}_{p} \cdot \dot{\vec{\beta}}\right) \int_{0}^{\pi} d \theta \sin \theta\left[\frac{\left(\beta^{2}-1\right)\left(1-\cos ^{2} \theta\right)+2(1-\beta \cos \theta)^{2}}{2 \rho^{4}}\right] \\
& +\frac{2 \pi\left(\dot{\vec{\varphi}}_{p} \cdot \vec{\beta}\right)(\vec{\beta} \cdot \dot{\vec{\beta}})}{\beta^{2}} \int_{0}^{\pi} d \theta \sin \theta\left[\frac{\left(\beta^{2}-1\right)\left(-1+3 \cos ^{2} \theta\right)+4 \beta \cos \theta(1-\beta \cos \theta)}{2 \rho^{4}}\right) .
\end{aligned}
$$

Finally, making the change $y=\cos \theta$, and defining

$$
c_{K}=\int_{-1}^{1} d y \frac{y^{K}}{(1-\beta y)^{4}}
$$

we get

$$
\begin{aligned}
I_{1}= & \pi\left(\dot{\vec{\beta}} \cdot \dot{\vec{\varphi}}_{p}\right)\left[\left(1+\beta^{2}\right)\left(c_{0}+c_{2}\right)-4 \beta c_{1}\right] \\
& +\pi\left(\vec{\beta} \cdot \dot{\vec{\varphi}}_{p}\right)(\vec{\beta} \cdot \dot{\vec{\beta}})\left[\frac{c_{0}-3 c_{2}+4 \beta c_{1}}{\beta^{2}}-\left(c_{0}+c_{2}\right)\right] .
\end{aligned}
$$

Now, we calculate $I_{2}=\int d \Omega \dot{\mathrm{h}}^{(2)} \cdot \overrightarrow{\mathrm{g}}^{\infty} \rho$. After elementary calculations, it can be written as

$$
I_{2}=\int d \Omega\left[\frac{1}{\rho^{3}} \dot{\vec{\beta}}^{2}\left(\hat{r}^{0} \cdot \vec{\varphi}_{p}\right)+\frac{2}{\rho^{4}}(\vec{\beta} \cdot \dot{\vec{\beta}})\left(\hat{r}^{0} \cdot \dot{\vec{\beta}}\right)\left(\hat{r}^{0} \cdot \vec{\varphi}_{p}\right)-\frac{\left(1-\beta^{2}\right)}{\rho^{5}}\left(\hat{r}^{0} \cdot \varphi_{p}\right)\left(\hat{r}^{0} \cdot \dot{\vec{\beta}}\right)^{2}\right]
$$

Considering the expressions $(\mathrm{A} 7 \mathrm{a})-(\mathrm{A} 7 \mathrm{c})$ and also

$$
\begin{gathered}
\int d \Omega g(\theta)\left(\hat{r}^{0} \cdot \overrightarrow{\mathrm{a}}\right)\left(\hat{r}^{0} \cdot \overrightarrow{\mathrm{b}}\right)\left(\overrightarrow{\mathrm{r}}^{0} \cdot \overrightarrow{\mathrm{c}}\right)=2 \pi \int_{0}^{\pi} d \theta \sin \theta g(\theta)\left\{\frac{1}{2} \sin ^{2} \theta \cos \theta\left[a_{z}(\overrightarrow{\mathrm{b}} \cdot \overrightarrow{\mathrm{c}})+b_{z}(\overrightarrow{\mathrm{a}} \cdot \overrightarrow{\mathrm{c}})+c_{z}(\overrightarrow{\mathrm{a}} \cdot \overrightarrow{\mathrm{b}})\right]\right. \\
\left.+a_{z} b_{z} c_{z}\left(\cos ^{3} \theta-\frac{3}{2} \sin ^{2} \theta \cos \theta\right)\right\} .
\end{gathered}
$$

We perform the integration over the angle $\varphi$ and obtain 


$$
\begin{gathered}
I_{2}=2 \pi \int_{0}^{\pi} d \theta \sin \theta\left\{\frac{1}{\rho^{3}} \dot{\vec{\beta}}^{2} \cos \theta \frac{\vec{\varphi}_{p} \cdot \vec{\beta}}{\beta}+\frac{2}{\rho^{4}}(\vec{\beta} \cdot \dot{\vec{\beta}})\left[\frac{1}{2}\left[\left(\vec{\varphi}_{p} \cdot \dot{\vec{\beta}}\right)-\frac{\left(\vec{\varphi}_{p} \cdot \vec{\beta}\right)(\vec{\beta} \cdot \dot{\vec{\beta}})}{\beta^{2}}\right]\right.\right. \\
\left.\quad+\frac{\cos ^{2} \theta}{2}\left[3 \frac{\left(\vec{\varphi}_{p} \cdot \vec{\beta}\right)(\vec{\beta} \cdot \dot{\vec{\beta}})}{\beta^{2}}-\left(\vec{\varphi}_{p} \cdot \dot{\vec{\beta}}\right)\right]\right] \\
-\frac{\left(1-\beta^{2}\right)}{\rho^{5}}\left[\frac{1}{2} \sin ^{2} \theta \cos \theta\left[\frac{\vec{\varphi}_{p} \cdot \vec{\beta}}{\beta} \dot{\vec{\beta}}^{2}+2 \frac{(\vec{\beta} \cdot \dot{\vec{\beta}})\left(\vec{\varphi}_{\dot{p}} \dot{\vec{\beta}}\right)}{\beta}\right]\right. \\
\left.\left.+\frac{\left(\vec{\varphi}_{p} \cdot \vec{\beta}\right)(\vec{\beta} \cdot \dot{\vec{\beta}})^{2}}{\beta^{3}}\left(\cos ^{3} \theta-\frac{3}{2} \sin ^{2} \theta \cos \theta\right)\right]\right\} .
\end{gathered}
$$

Making again the change $\cos \theta=y$ and defining now

$$
\begin{aligned}
& b_{k}=\int_{-1}^{1} d y \frac{y^{k}}{(1-\beta y)^{5}}, \\
& d_{k}=\int_{-1}^{1} d y \frac{y^{k}}{(1-\beta y)^{3}},
\end{aligned}
$$

$I_{2}$ can be written

$$
\begin{aligned}
& I_{2}=2 \pi\left[\left(\vec{\beta} \cdot \vec{\varphi}_{p}\right) \dot{\vec{\beta}}^{2}\left[\frac{d_{1}}{\beta}-\frac{\left(b_{1}-b_{2}\right)\left(1-\beta^{2}\right)}{2 \beta}\right]+\left(\vec{\beta} \cdot \vec{\varphi}_{p}\right)(\vec{\beta} \cdot \dot{\vec{\beta}})^{2}\left[-\frac{c_{0}}{\beta^{2}}+\frac{3 c_{2}}{\beta^{2}}-\frac{\left(1-\beta^{2}\right)}{\beta^{3}}\left(\frac{5}{2} b_{3}-\frac{3}{2} b_{1}\right)\right]\right. \\
&\left.+\left(\vec{\varphi}_{p} \cdot \dot{\vec{\beta}}\right)(\vec{\beta} \cdot \dot{\vec{\beta}})\left[c_{0}-c_{2}-\frac{\left(1-\beta^{2}\right)}{\beta}\left(b_{1}-b_{3}\right)\right]\right]
\end{aligned}
$$

It remains to calculate the integrals $b_{k}, c_{k}$, and $d_{k}$. For that, it is easy to prove the relations

$$
\begin{aligned}
& b_{k}=\frac{1}{4 \beta\left(1-\beta^{2}\right)^{4}}\left[(1+\beta)^{4}-(-1)^{k}(1-\beta)^{4}\right]-\frac{k}{4 \beta} c_{k-1}, \\
& c_{k}=\frac{1}{3 \beta\left(1-\beta^{2}\right)^{3}}\left[(1+\beta)^{3}-(-1)^{k}(1-\beta)^{3}\right]-\frac{k}{3 \beta} d_{k-1},
\end{aligned}
$$

which allow us to obtain the following values:

$$
\begin{aligned}
& d_{0}=2 \gamma^{4}, \quad d_{1}=2 \beta \gamma^{4}, \quad c_{0}=\frac{2}{3} \gamma^{6}\left(3+\beta^{2}\right), \quad c_{1}=\frac{8}{3} \gamma^{6} \beta, \\
& c_{2}=\frac{2}{3} \gamma^{6}\left(1+3 \beta^{2}\right), \quad b_{0}=2 \gamma^{8}\left(1+\beta^{2}\right), \quad b_{1}=\frac{2}{3} \beta \gamma^{8}\left(5+\beta^{2}\right), \\
& b_{2}=\frac{2}{3} \gamma^{8}\left(1+5 \beta^{2}\right), \quad b_{3}=2 \beta \gamma^{8}\left(1+\beta^{2}\right) .
\end{aligned}
$$

Finally, substituting (A16) into (A9) and (A15) we obtain

$$
\begin{aligned}
\int d \Omega \overrightarrow{\mathrm{h}}_{p}^{\infty} \cdot \overrightarrow{\mathrm{g}}^{\infty}\left(1-\vec{\beta} \cdot \hat{r}^{0}\right)= & \pi\left(\dot{\vec{\beta}} \cdot \dot{\vec{\varphi}}_{p}\right) \frac{8}{3} \gamma^{2}+\pi\left(\vec{\beta} \cdot \dot{\vec{\varphi}}_{p}\right)(\vec{\beta} \cdot \dot{\vec{\beta}}) \frac{8}{3} \gamma^{4} \\
& +2 \pi\left(\vec{\beta} \cdot \vec{\varphi}_{p}\right) \dot{\vec{\beta}}^{2} \frac{4}{3} \gamma^{4}+2 \pi\left(\vec{\beta} \cdot \vec{\varphi}_{p}\right)(\vec{\beta} \cdot \dot{\vec{\beta}})^{2} \frac{4}{3} \gamma^{6}=\frac{8 \pi}{3} \Lambda,
\end{aligned}
$$

which is the relation we were looking for. 


\section{APPENDIX B}

We must calculate the integral

$$
L=\int d \Omega \cos \left[\frac{\omega}{c} \hat{r}^{0} \cdot\left(\overrightarrow{\mathrm{r}}-\overrightarrow{\mathrm{r}}^{-u}\right)-\omega u\right]\left[\vec{\varphi}_{p} \cdot\left(I-\hat{r}^{0} \hat{r}^{0}\right) \cdot \vec{\beta}^{-u}+\left(\vec{\varphi}_{p} \cdot \hat{r}^{0}\right)\left(\vec{\beta} \cdot \vec{\beta}^{-u}\right)-\left(\vec{\varphi}_{p} \cdot \vec{\beta}^{-u}\right)\left(\vec{\beta}^{0} \cdot \hat{r}^{0}\right)\right]
$$

Let us call

$$
\overrightarrow{\mathrm{V}}_{\varphi}=\vec{\varphi}_{p}\left(\vec{\beta} \cdot \vec{\beta}^{-u}\right)-\left(\vec{\varphi}_{p} \cdot \vec{\beta}^{-u}\right) \vec{\beta} .
$$

Then (B1) can be written

$$
L=\int d \Omega \cos \left[\frac{\omega}{c} \hat{r}^{0} \cdot\left(\overrightarrow{\mathrm{r}}-\overrightarrow{\mathrm{r}}^{-u}\right)-\omega u\right]\left[\vec{\varphi}_{p} \cdot\left(I-\hat{r}^{0} \hat{r}^{0}\right) \cdot \vec{\beta}^{-u}+\hat{r}^{0} \cdot \overrightarrow{\mathrm{V}}_{\varphi}\right] .
$$

Choosing the $0 Z$ axis in the direction of $\vec{r}-\vec{r}^{-u}$ and using the definitions (5.37) and the relations (A7), we perform the integration over the angle $\varphi$ resulting in

$$
\begin{aligned}
L=\int_{0}^{\pi} \sin \theta d \theta \cos [\omega \alpha \cos \theta-\omega u] 2 \pi & {\left[\left(\vec{\varphi}_{p} \cdot \vec{\beta}^{-u}\right)\left(\frac{1+\cos ^{2} \theta}{2}\right]\right.} \\
& \left.+\left(\vec{\varphi}_{p} \cdot \hat{n}_{u}\right)\left(\vec{\beta}^{-u} \cdot \hat{n}_{u}\right)\left(\frac{1-3 \cos ^{2} \theta}{2}\right)+V_{\varphi z} \cos \theta\right] .
\end{aligned}
$$

Now, taking into account that

$$
\int_{0}^{\pi} \sin \theta \cos [\omega \alpha \cos \theta] \cos \theta=0
$$

and

$$
\int_{0}^{\pi} \sin \theta \sin [\omega \alpha \cos \theta] f\left(\cos ^{2} \theta\right)=0,
$$

the result for $L$

$$
\begin{aligned}
L=2 \pi \int_{0}^{\pi} \sin \theta d \theta\{ & \cos (\omega u) \cos (\omega \alpha \cos \theta)\left[\left(\vec{\varphi}_{p} \cdot \vec{\beta}^{-u}\right)\left(\frac{1+\cos ^{2} \theta}{2}\right)+\left(\vec{\varphi}_{p} \cdot \hat{n}_{u}\right)\left(\vec{\beta}^{-u} \hat{n}_{u}\right)\left(\frac{1-3 \cos ^{2} \theta}{2}\right)\right] \\
& \left.+\sin (\omega u) \sin (\omega \alpha \cos \theta)\left(\overrightarrow{\mathbf{V}}_{\varphi} \cdot \hat{n}_{u}\right) \cos \theta\right\} .
\end{aligned}
$$

We make again the change $\cos \theta=\mathrm{y}$ and consider the following integrals:

$$
\begin{aligned}
& C_{0}=\int_{-1}^{1} d y \cos (\omega \alpha y)=2 \frac{\sin \omega \alpha}{\omega \alpha}, \\
& C_{2}=\int_{-1}^{1} d y \cos (\omega \alpha y) y^{2}=2\left[\frac{\sin \omega \alpha}{\omega \alpha}+2 \frac{\cos \omega \alpha}{\omega^{2} \alpha^{2}}-2 \frac{\sin \omega \alpha}{\omega^{3} \alpha^{3}}\right], \\
& S_{1}=\int_{-1}^{1} d y y \sin (\omega \alpha y)=-2\left[\frac{\cos \omega \alpha}{\omega \alpha}-\frac{\sin \omega \alpha}{\omega^{2} \alpha^{2}}\right] .
\end{aligned}
$$

Note that these expressions are all well defined in the case that $\alpha \rightarrow 0$.

Now, (B2) can be written

$$
L=2 \pi\left\{\cos (\omega u)\left[\left(\vec{\varphi}_{p} \cdot \vec{\beta}^{-u}\right) \frac{1}{2}\left(C_{0}+C_{2}\right)+\left(\vec{\varphi}_{p} \cdot \hat{n}_{u}\right)\left(\vec{\beta}^{-u} \cdot \hat{n}_{u}\right) \frac{1}{2}\left(C_{0}-3 C_{2}\right)\right]+\sin (\omega u)\left(\overrightarrow{\mathrm{V}}_{\varphi} \cdot \hat{n}_{u}\right) S_{1}\right\} .
$$


Substituting the values of $C_{0}, C_{2}$, and $S_{1}$, and regrouping terms, we obtain finally

$$
\begin{aligned}
L= & 4 \pi \cos (\omega u) \frac{\sin (\omega \alpha)}{\omega \alpha} P[\varphi](u) \\
& +4 \pi \cos (\omega u)\left(\frac{\cos \omega \alpha}{\omega^{2} \alpha^{2}}-\frac{\sin \omega \alpha}{\omega^{3} \alpha^{3}}\right) Q[\varphi](u) \\
& +4 \pi \sin (\omega u)\left(\frac{\sin \omega \alpha}{\omega^{2} \alpha^{2}}-\frac{\cos \omega \alpha}{\omega \alpha}\right) R[\varphi](u),
\end{aligned}
$$

where $P, Q$, and $R$ are given in (5.36a) $-(5.36 \mathrm{c})$. This proves finally (5.34).

\section{APPENDIX C}

We want to calculate (5.34) in the limit $\epsilon \rightarrow 0$. It will be useful in the following to know the first terms of an expansion in the parameter $u$, of the several functions which appear in the integrals. An easy calculation gives the following expressions:

$$
\begin{aligned}
& \alpha=v \beta u \Pi(u), \\
& \Pi(u)=\left[1-\frac{\vec{\beta} \cdot \dot{\vec{\beta}}}{\beta^{2}} u+O\left(u^{2}\right)\right], \\
& \dot{\alpha}=v \beta\left[1-\frac{\vec{\beta} \cdot \dot{\vec{\beta}}}{\beta^{2}} u+O\left(u^{2}\right)\right], \\
& \hat{n}_{u}=v\left[\frac{\vec{\beta}}{\beta}+\frac{1}{2} u \vec{\lambda}+O\left(u^{2}\right)\right], \\
& \vec{\lambda}=\frac{\vec{\beta} \cdot \dot{\vec{\beta}}}{\beta^{3}} \vec{\beta}-\frac{\dot{\vec{\beta}}}{\beta}, \\
& P[\varphi]=-\frac{1}{2} u \xi[\varphi]+O\left(u^{2}\right), \\
& \xi[\varphi]=\vec{\varphi}_{p} \cdot \dot{\vec{\beta}}-\frac{\left(\vec{\varphi}_{p} \cdot \vec{\beta}\right)(\vec{\beta} \cdot \dot{\vec{\beta}})}{\beta^{2}}, \\
& \frac{P[\varphi]}{\alpha}=v\left[-\frac{1}{2 \beta} \xi[\varphi]+O(u)\right], \\
& R[\varphi]=v u\left[\frac{1}{2} \beta \xi[\varphi]+O(u)\right], \\
& \frac{R[\varphi]}{\alpha}=\frac{1}{2} \xi[\varphi]+O(u), \\
& Q[\varphi]=Q_{0}+u \dot{Q}_{0}+O\left(u^{2}\right), \\
& Q_{0}=-2 \vec{\varphi}_{p} \cdot \vec{\beta}, \\
& \dot{Q}_{0}=\frac{1}{2}\left[\left(\vec{\varphi}_{p} \cdot \dot{\vec{\beta}}\right)+3 \frac{\left(\vec{\varphi}_{p} \cdot \vec{\beta}\right)(\vec{\beta} \cdot \dot{\vec{\beta}})}{\beta^{2}}\right],
\end{aligned}
$$

and

$$
v=\operatorname{sgn}(u) \text {. }
$$

Let us calculate each of the integrals in (5.34) separately.

$$
\text { (a) } \begin{aligned}
K_{1}= & \int_{0}^{\infty} I_{1}(u ; \epsilon) d u \\
= & \int_{0}^{\infty} d u \frac{P[\varphi](u)}{\alpha(u)} \\
& \quad \times \int_{0}^{\infty} d \omega \omega e^{-\epsilon \omega} \cos (\omega u) \sin (\omega \alpha)
\end{aligned}
$$

The integration over $\omega$ gives

$$
\begin{aligned}
K_{1}=\int_{0}^{\infty} d u \frac{P[\varphi](u)}{\alpha(u)} \epsilon & {\left[\frac{u+\alpha}{\left[\epsilon^{2}+(u+\alpha)^{2}\right]^{2}}\right.} \\
& \left.-\frac{u-\alpha}{\left[\epsilon^{2}+(u-\alpha)^{2}\right]^{2}}\right] .
\end{aligned}
$$

Let us introduce a parameter $\delta>0$ and write

$$
K_{1}=\int_{0}^{\delta} I_{1}(u ; \epsilon) d u+\int_{\delta}^{\infty} I_{1}(u ; \epsilon) d u
$$

We shall prove that the second integral vanishes $\epsilon \rightarrow 0$ independently of the value of $\delta$. Note firstly that, for any value of $\epsilon, I_{1}$ goes to zero as $u \rightarrow \infty$, at least faster than $1 / u^{3}$ and then, the integral exists. On the other hand, choosing $\epsilon<<$, then

$$
\begin{aligned}
\int_{\delta}^{\infty} & I_{1}(u ; \epsilon) d u \\
& \sim \epsilon \int_{\delta}^{\infty}\left[\frac{P}{\alpha}\right](u)\left(\frac{1}{(u+\alpha)^{3}}-\frac{1}{(u-\alpha)^{3}}\right) \\
& =-2 \epsilon \int_{\delta}^{\infty} P[\varphi](u) \frac{\left(\alpha^{2}+3 u^{2}\right)}{\left(u^{2}-\alpha^{2}\right)^{3}} .
\end{aligned}
$$

The integrand of this expression is continuous $\forall u$, and behaves like $1 / u^{4}$ as $u \rightarrow \infty$. Therefore the integral of (C4) is finite and then

$$
\int_{\delta}^{\infty} I_{1}(u ; \epsilon) d u=r_{1}(\epsilon) \underset{\epsilon \rightarrow 0}{\rightarrow} 0 .
$$

Consider now the first integral of (C3). Let us define

$$
\begin{aligned}
& \phi_{1}(\epsilon ; \delta)=\epsilon \int_{0}^{\delta} I_{1}(u ; \epsilon) d u, \\
& \phi_{1}(0 ; \delta)=\lim _{\epsilon \rightarrow 0} \epsilon \int_{0}^{\delta} I_{1}(u ; \epsilon) d u .
\end{aligned}
$$

Obviously, $\phi_{1}(\epsilon ; \delta) \in C^{1}(0, \infty)$ that is, it is continuous and has a continuous derivative for $\epsilon \neq 0$. 
We shall prove that this is also true for $\epsilon=0$.

Let us calculate (C6b). For this, we make the change $u=\epsilon x$, so $v=\operatorname{sgn} x$. Taking (C1a) into account,

$$
\phi_{1}(\epsilon ; \delta)=\int_{0}^{\delta / \epsilon} d x x \Gamma_{1}(\epsilon x) F_{1}(x ; \Pi(\epsilon x)),
$$

where

$$
\Gamma_{1}(u)=\frac{P[\varphi](u)}{\alpha},
$$

$$
\begin{aligned}
F_{1}(x ; \Pi)= & \frac{1+v \beta \Pi}{\left[1+x^{2}(1+v \beta \Pi)^{2}\right]^{2}} \\
& -\frac{1-v \beta \Pi}{\left[1+x^{2}(1-v \beta \Pi)^{2}\right]^{2}},
\end{aligned}
$$

$\Gamma_{1}(u) \cdot F(x ; \Pi(u)) \in C^{1}$ as a function of $u$, so we can apply the theorem of the mean value,

$\Gamma_{1}(u) F_{1}(x, \Pi(u))-\Gamma_{1}(0+) F_{1}(x ; \Pi(0+))$

$$
=\left.u\left(\frac{\partial}{\partial u} \Gamma_{1}(u) F_{1}(x ; \Pi(u))\right)\right|_{u=u^{\prime}},
$$

where $0 \leq u^{\prime}<u$. Then (C7) can be written

$$
\phi_{1}(\epsilon ; \delta)=\int_{0}^{\delta / \epsilon} d x \times \Gamma_{1}(0+) F_{1}(x ; \Pi(0+))+\int_{0}^{\delta / \epsilon} d x x \epsilon x\left(\frac{\partial}{\partial u}\left[\Gamma_{1}(u) F_{1}(x ; \Pi(u))\right]\right]_{u=\epsilon^{\prime} x} . \quad 0 \leq \epsilon^{\prime} \leq \epsilon
$$

(C10)

The second integral of (C10) will vanish as $\epsilon \rightarrow 0$. To see that note that

$\dot{\Gamma}_{1}(\epsilon x) F_{1}(x ; \Pi(\epsilon x))+\Gamma_{1}(\epsilon x) \frac{\partial F_{1}}{\partial \Pi}(x ; \Pi(\epsilon x)) \dot{\Pi}(\epsilon x)$

is continuous in $[0, \infty)$ and behaves at least as $1 / x^{2}$ in the limit $x \rightarrow \infty$. This is true even for $\epsilon=0$, so the integral

$$
\int_{0}^{\infty} d x x^{2}\left[\frac{\partial}{\partial u}\left[\Gamma_{1}(u) F_{1}(x ; \Pi(u))\right]\right]_{u=0}
$$

is finite. As it is multiplied by $\epsilon$, it goes to zero as $\epsilon \rightarrow 0$. Therefore

$$
\begin{aligned}
\phi_{1}(0 ; \delta) & =\lim _{\epsilon \rightarrow 0} \phi_{1}(\epsilon ; \delta) \\
& =\lim _{\epsilon \rightarrow 0} \int_{0}^{\delta / \epsilon} d x x \Gamma_{1}(0+) F_{1}(x ; 1) \\
& =\Gamma_{1}(0+) \int_{0}^{\infty} x F_{1}(x ; 1) d x
\end{aligned}
$$

Let

$$
\phi_{1}(0 ; \delta)=T_{1}[\varphi] .
$$

The integral in (C11) is finite because $x F_{1}$ is continuous in $[0, \infty)$ and goes to zero like $1 / x^{3}$ as $x$ goes to $\infty$. The calculation of it will be made below.

Now we are going to prove that the first derivative of $\phi_{2}$ has a limit as $\epsilon \rightarrow 0$. From (C7) we have

$$
\begin{aligned}
\frac{\partial \phi_{1}}{\partial \epsilon}= & -\frac{\delta}{\epsilon^{2}}\left[\frac{\delta}{\epsilon}\right] \Gamma_{1}(\delta) F_{1}\left(\frac{\delta}{\epsilon} ; \Pi(\delta)\right) \\
& +\int_{0}^{\delta / \epsilon} d x x^{2}\left[\frac{\partial}{\partial u} \Gamma_{1}(u) F_{1}(x ; \Pi(u))\right]_{u=\epsilon x}
\end{aligned}
$$

(C13)

This expression is well defined if $\epsilon \neq 0$. We take now the limit $\epsilon \rightarrow 0$. As

$$
\begin{aligned}
F_{1}\left[\frac{\delta}{\epsilon} ; \Pi(\delta)\right]= & \frac{1+v \beta \Pi(\delta)}{\left[1+\frac{\delta^{2}}{\epsilon^{2}}(1+v \beta \Pi(\delta))^{2}\right]^{2}} \\
& -\frac{1-v \beta \Pi(\delta)}{\left[1+\frac{\delta^{2}}{\epsilon^{2}}(1-v \beta \Pi(\delta))^{2}\right]^{2}} \\
= & \epsilon^{4}\left[\frac{[1+v \beta \Pi(\delta)]}{\left[\epsilon^{2}+\delta^{2}(1+v \beta \Pi(\delta))^{2}\right]^{2}}\right. \\
& \left.-\frac{[1-v \beta \Pi(\delta)]}{\left[\epsilon^{2}+\delta^{2}(1-v \beta \Pi(0))^{2}\right]^{2}}\right] .
\end{aligned}
$$

The first term of the right-hand side of (C13) vanishes as $\epsilon \rightarrow 0$. For the second term we make an analysis similar to the one made above. Let

$$
g(x, u)=\frac{\partial}{\partial u} \Gamma_{1}(u) F_{1}(x ; \Pi(u)) .
$$

Using again the mean value theorem 


$$
\begin{aligned}
\int_{0}^{\delta / \epsilon} d x x^{2} g(x, \epsilon x) & -\int_{0}^{\delta / \epsilon} d x x^{2} g(x, 0+) \\
= & \left.\int_{0}^{\delta / \epsilon} d x x^{2} \epsilon x \frac{\partial g}{\partial u}\right|_{u=\epsilon^{\prime} x} \\
= & \left.\epsilon \int_{0}^{\delta / \epsilon} d x x^{3} \frac{\partial g}{\partial u}\right|_{u=\epsilon^{\prime} x} .
\end{aligned}
$$

Now $\partial g / \partial u$ behaves like $1 / x^{4}$, as $x \rightarrow \infty$ and the integration gives rise to a term of type $\ln \epsilon$, if $\epsilon \ll \delta$, then (C14) results in $\epsilon \ln \epsilon$ which goes to zero as $\epsilon \rightarrow 0$. Therefore

$$
\frac{\partial \phi_{1}}{\partial \epsilon}=\int_{0}^{\delta / \epsilon} d x x^{2} g(x, 0+)+\bar{r}_{2}(\epsilon)
$$

and

$$
\begin{aligned}
\lim _{\epsilon \rightarrow 0} \frac{\partial \phi_{1}}{\partial \epsilon} & =\int_{0}^{\infty} d x x^{2} g(x, 0+) \\
& =\left.\frac{\partial \phi_{1}}{\partial \epsilon}\right|_{\epsilon=0} .
\end{aligned}
$$

We have then shown that

$$
\frac{\partial \phi_{1}}{\partial \epsilon}=\left.\frac{\partial \phi_{1}}{\partial \epsilon}\right|_{\epsilon=0}+r_{2}(\epsilon)=D_{1}+r_{2}(\epsilon),
$$

where

$$
r_{2}(\epsilon) \underset{\epsilon \rightarrow 0}{\rightarrow} 0 .
$$

Once this is made, we can apply the mean value theorem to the function $\phi_{1}$,

$$
\phi_{1}(\epsilon ; \delta)=\phi_{1}(0 ; \delta)+\left.\epsilon \frac{\partial \phi_{1}}{\partial \epsilon}\right|_{\epsilon^{\prime}<\epsilon},
$$

and taking (C12) and (C15) into account,

$$
\phi_{1}(\epsilon ; \delta)=T_{1}[\varphi]+\epsilon D_{1}+\epsilon r_{2}(\epsilon)
$$

and finally

$$
\begin{aligned}
K_{1}=\frac{1}{\epsilon} \phi_{1}(\epsilon ; \delta)+r_{1}(\epsilon)= & \frac{1}{\epsilon} T_{1}[\varphi]+D_{1} \\
& +r_{2}(\epsilon)+r_{1}(\epsilon) .
\end{aligned}
$$

(C17)

If we consider now the expression of $K_{1}$ but integrated over values of $u<0$,

$$
\bar{K}_{1}=\int_{-\infty}^{0} I_{1}(u ; \epsilon) d u,
$$

and making the same calculations, we obtain

$$
\bar{K}_{1}=\frac{1}{\epsilon} \bar{T}_{1}[\varphi]+\bar{D}_{1}+\bar{r}_{2}+\bar{r}_{1},
$$

where

$$
\begin{aligned}
& \bar{T}_{1}=\Gamma_{1}(0-) \int_{-\infty}^{0} d x x F_{1}(x ; 1) \\
& \bar{D}_{1}=\int_{-\infty}^{0} d x x^{2} g(x ; 0-)
\end{aligned}
$$

Now, from $(\mathrm{C} 8)$ and $(\mathrm{C} 1 \mathrm{~h})$ we find that

$$
\Gamma_{1}(0+)=-\frac{1}{2 \beta} \xi[\varphi]
$$

and

$$
\Gamma_{1}(0-)=\frac{1}{2 \beta} \xi[\varphi]
$$

Also from (C9) we see that $F_{1}$ is an odd function and then it is immediately clear that $\bar{T}_{1}=-T_{1}$.

On the other hand, from $(\mathrm{C} 1 \mathrm{~h})$ again, $\dot{\Gamma}_{1}(0+)=-\dot{\Gamma}_{1}(0-)$ and, as $F$ is odd, $g(x, 0+)=g(-x, 0-)$. Then $\bar{D}_{1}=D_{1}$. With all this, adding (C17) and (C18) we find that

$$
\begin{aligned}
K_{1}+\bar{K}_{1}=\int_{-\infty}^{+\infty} I_{1}(u ; \epsilon) d u & =2 D_{1}+r_{1}+r_{2}+\bar{r}_{1}+\bar{r}_{2} \\
& =\int_{-\infty}^{+\infty} I_{1}(u ; 0) d u+\bar{R}_{1}(\epsilon)
\end{aligned}
$$

and finally

$$
K_{1}=\frac{1}{\epsilon} T_{1}[\varphi]+\frac{1}{2} \int_{-\infty}^{+\infty} I_{1}(u ; 0) d u+R_{1}(\epsilon)
$$

with $R_{1}(\epsilon) \underset{\epsilon \rightarrow 0}{\rightarrow} 0$.

$$
\text { (b) } \begin{aligned}
K_{3}= & \int_{0}^{\infty} I_{3}(u ; \epsilon) d u \\
=\int_{0}^{\infty} d u R[\varphi](u) & \\
& \times \int_{0}^{\infty} d \omega \omega e^{-\epsilon \omega} \sin (\omega u)\left[\frac{\sin (\omega \alpha)}{\omega \alpha}\right. \\
& -\cos (\omega \alpha)] .
\end{aligned}
$$

A first integration over $\omega$ gives

$$
\begin{gathered}
I_{3}=\frac{R[\varphi](u)}{\alpha^{2}}\left[-\frac{1}{2}\right] \epsilon\left[\frac{1}{\epsilon^{2}+(u+\alpha)^{2}}-\frac{1}{\epsilon^{2}+(u-\alpha)^{2}}\right] \\
-\frac{R[\varphi](u)}{\alpha} \epsilon\left[\frac{u+\alpha}{\left[\epsilon^{2}+(u+\alpha)^{2}\right]^{2}}+\frac{u-\alpha}{\left[\epsilon^{2}+(u-\alpha)^{2}\right]^{2}}\right] .
\end{gathered}
$$

Introducing again the parameter $\delta$, and choosing $\epsilon<\delta$ we have 


$$
\begin{aligned}
\int_{\delta}^{\infty} I_{3}(u ; \epsilon) d u & \sim \epsilon \int_{\delta}^{\infty} d u \frac{R[\varphi]}{\alpha}\left[\left(\frac{1}{(u+\alpha)^{2}}-\frac{1}{(u-\alpha)^{2}}\right)\left[-\frac{1}{2 \alpha}\right)-\left(\frac{1}{(u+\alpha)^{3}}+\frac{1}{(u-\alpha)^{3}}\right)\right] \\
& =\epsilon \int_{\delta}^{\infty} R[\varphi](u)(-8) \frac{u \alpha}{\left(u^{2}-\alpha^{3}\right)^{3}},
\end{aligned}
$$

which vanishes as $\epsilon \rightarrow 0$ because the integrand is continuous in $[\delta, \infty)$ and behaves like $1 / u^{5}$ as $u \rightarrow \infty$. Therefore,

$$
K_{3}=\int_{0}^{\delta} I_{3}(u ; \epsilon) d u+r_{3}(\epsilon) .
$$

We define now

$$
\phi_{3}(\epsilon ; \delta)=\epsilon \int_{0}^{\delta} I_{3}(u ; \epsilon) d u,
$$

$$
\phi_{3}(0 ; \delta)=\lim _{\epsilon \rightarrow 0} \epsilon \int_{0}^{\delta} I_{3}(u ; \epsilon) d u .
$$

Again, $\phi_{3} \in C^{1}(0, \infty)$ and we shall prove that also $\phi_{3} \in C^{1}[0, \infty)$. We show first that (C21b) exists.

Let us make again the change $u=\epsilon x$; it results in

$$
\phi_{3}(\epsilon ; \delta)=\int_{0}^{\delta / \epsilon} x \Gamma_{3}(\epsilon x) F_{3}(x ; \Pi(\epsilon x)) d x,
$$

where

$$
\begin{aligned}
& \Gamma_{3}(u)=\frac{R[\varphi](u)}{\alpha}, \\
& F_{3}(x ; \Pi)=-\frac{1}{2 v \beta x^{2} \Pi}\left[\frac{1}{1+x^{2}(1+v \beta \Pi)^{2}}-\frac{1}{1+x^{2}(1-v \beta \Pi)^{2}}\right]-\left[\frac{(1+v \beta \Pi)}{\left[1+x^{2}(1+v \beta \Pi)^{2}\right]^{2}}+\frac{(1-v \beta \Pi)}{\left[1+x^{2}(1-v \beta \Pi)^{2}\right]^{2}}\right] .
\end{aligned}
$$

We make now the same calculations as we made for $K_{1}$. Note that, again $\Gamma_{3}(0+)$ is finite, $F_{3}(x, \Pi)$ behaves like $1 / x^{4}$ as $x \rightarrow \infty$ and $\Gamma_{3}(u) . F_{3}(x ; \Pi(u))$ is continuous for $u \in[0, \infty)$. We only give here the result

$$
K_{3}=\frac{1}{\epsilon} T_{3}[\varphi]+\frac{1}{2} \int_{-\infty}^{+\infty} I_{3}(u ; 0) d u+R_{3}(\epsilon)
$$

with

$$
T_{3}[\varphi]=\Gamma_{3}(0+) \int_{0}^{\infty} x F_{3}(x ; 1) d x .
$$

Now, we are going to calculate the sum $T_{1}+T_{3}$. Substituting (C1h) and (C9) in (C11), and (C1j) and (C24) in (C26), adding (C11) and (C26) and regrouping terms, we obtain

$$
\begin{aligned}
T_{1}+T_{3}=-\frac{\xi[\varphi]}{2 \beta}[ & \int_{0}^{\infty} d x x\left(\frac{(1+\beta)^{2}}{\left[1+x^{2}(1+\beta)^{2}\right]^{2}}-\frac{(1-\beta)^{2}}{\left[1+x^{2}(1-\beta)^{2}\right]^{2}}\right] \\
& \left.+\int_{0}^{\infty} d x \frac{1}{2 x}\left(\frac{1}{1+x^{2}(1+\beta)^{2}}-\frac{1}{1+x^{2}(1-\beta)^{2}}\right]\right] .
\end{aligned}
$$

Using the integrals

$$
S_{1}(a)=\int d x \frac{x a^{2}}{\left(1+x^{2} a^{2}\right)^{2}}=-\frac{1}{2\left(1+x^{2} a^{2}\right)}, \quad S_{2}(a)=\int d x \frac{1}{x\left(1+x^{2} a^{2}\right)}=\ln x-\frac{1}{2} \ln \left(1+x^{2} a^{2}\right),
$$

the integration in (C27) is immediate and the result obtained is 


$$
T_{1}[\varphi]+T_{3}[\varphi]=\frac{\xi[\varphi]}{4 \beta} \ln \frac{1+\beta}{1-\beta} .
$$

(c) $K_{2}=\int_{0}^{\infty} I_{2}(u ; \epsilon) d u=\int_{0}^{\infty} d u Q[\varphi](u) \int_{0}^{\infty} d w w^{2} e^{-\epsilon w} \sin (w u)\left[\cos (w \alpha) / w^{2} \alpha^{2}-\sin (w \alpha) / w^{3} \alpha^{3}\right]$. The integration over $w$ gives

$$
I_{2}(u ; \epsilon)=\frac{Q_{2}[\varphi](u)}{2 \alpha^{2}}\left[\frac{\epsilon}{\epsilon^{2}+(u+\alpha)^{2}}+\frac{\epsilon}{\epsilon^{2}+(u-\alpha)^{2}}-\frac{1}{\alpha}\left[\arctan \frac{u+\alpha}{\epsilon}-\arctan \frac{u-\alpha}{\epsilon}\right)\right] .
$$

We introduce again the parameter $\delta$ and choose $\epsilon \ll \delta$. Considering that $\arctan 1 / \epsilon \sim \pi / 2-\epsilon+\frac{1}{2} \epsilon^{3}+\cdots$,

$$
\begin{aligned}
\int_{\delta}^{\infty} I_{2}(u ; \epsilon) d u & \sim \epsilon \int_{\delta}^{\infty} \frac{Q}{2 \alpha^{2}}\left[\frac{1}{(u+\alpha)^{2}}+\frac{1}{(u-\alpha)^{2}}-\frac{2}{\left(u^{2}-\alpha^{2}\right)}+O\left(\frac{1}{u^{4}}\right)\right] d u \\
& =\epsilon \int_{\delta}^{\infty} 2 Q\left[\frac{1}{\left(u^{2}-\alpha^{2}\right)^{2}}+O\left(\frac{1}{u^{4}}\right)\right] d u
\end{aligned}
$$

The integral is finite, and then $\int_{\delta}^{\infty} I_{2}(u ; \epsilon) d u \underset{\epsilon \rightarrow 0}{\rightarrow} 0$, therefore

$$
K_{2}=\int_{0}^{\infty} I_{2}(u ; \epsilon) d u+r_{4}(\epsilon) .
$$

Now, we define

$$
\begin{aligned}
& \phi_{2}(\epsilon ; \delta)=2 \beta^{2} \epsilon^{2} \int_{0}^{\delta} I_{2}(u ; \epsilon) d \epsilon, \\
& \phi_{2}(0 ; \delta)=2 \beta^{2} \lim _{\epsilon \rightarrow 0} \epsilon^{2} \int_{0}^{\delta} I_{2}(u ; \epsilon) d \epsilon .
\end{aligned}
$$

(C30b) where

In this case we are interested in considering up to the second derivative. We shall prove that $\phi_{2} \in C^{2}[0, \infty)$ in the variable $\epsilon$. For that let us begin by showing that (C30b) exist. Making again the change $u=\epsilon x$ we have

$$
\phi_{2}(\epsilon ; \delta)=\int_{0}^{\delta / \epsilon} Q[\varphi](\epsilon x) F_{2}(x ; \Pi(\epsilon x)) d x,
$$

$$
\begin{aligned}
F_{2}(x, \Pi)=\frac{1}{x^{2} \Pi^{2}} & \left(\frac{1}{1+x^{2}(1+v \beta \Pi)^{2}}+\frac{1}{1+x^{2}(1-v \beta \Pi)^{2}}\right. \\
& \left.-\frac{1}{v \beta \Pi x}[\arctan x(1+v \beta \Pi)-\arctan x(1-v \beta \Pi)]\right) .
\end{aligned}
$$

From that expression we see that $F_{2}$ is finite in the limit $\epsilon \rightarrow 0$ and behaves like $1 / x^{4}$ as $x \rightarrow \infty$. We apply to the integrand of (C31), the mean value theorem, and obtain

$$
\int_{0}^{\delta / \epsilon} Q(\epsilon x) F_{2}(x, \Pi(\epsilon x)) d x=\int_{0}^{\delta / \epsilon} Q(0+) F_{2}(x ; 1) d x+\left.\epsilon \int_{0}^{\delta / \epsilon} d x x \frac{\partial}{\partial u}\left[Q(u) F_{2}(x, \Pi(u))\right]\right|_{u=\epsilon^{\prime} x}
$$

The last integral is finite when we put $\epsilon=0$ because the integrand is of order $1 / x^{3}$ as $x \rightarrow \infty$. Then, equally as in the cases above,

$$
\lim _{\epsilon \rightarrow 0} \int_{0}^{\delta / \epsilon} Q(\epsilon x) F_{2}(x ; \Pi(\epsilon x)) d x=\lim _{\epsilon \rightarrow 0} \int_{0}^{\delta / \epsilon} Q(0+) F_{2}(x ; 1) d x=\int_{0}^{\infty} Q(0+) F_{2}(x ; 1) d x .
$$

That expression can be integrated immediately, substituting (C32) and using the integrals

$$
S_{3}(a)=\int d x \frac{1}{x^{2}\left(1+x^{2} a^{2}\right)}=-\frac{1}{x}-a \arctan (a x) ;
$$




$$
S_{4}(a)=\int d x \frac{1}{x^{3}} \arctan (x a)=-\frac{1}{2 x^{2}} \arctan (x a)+\frac{a}{2} S_{3}(a) .
$$

The result is

$$
\phi_{2}(0 ; \delta)=\lim _{\epsilon \rightarrow 0} \phi_{2}(\epsilon ; \delta) \equiv 0 .
$$

We calculate now the first derivative in $\epsilon \rightarrow 0$. From (C31) we have

$$
\frac{\partial \phi_{2}}{\partial \epsilon}=-\frac{\delta}{\epsilon^{2}} Q[\varphi](\delta) F_{2}\left(\frac{\delta}{\epsilon} ; \Pi(\delta)\right]+\int_{0}^{\delta / \epsilon} d x x\left[\dot{Q}[\varphi](\epsilon x) F_{2}(x ; \Pi(\epsilon x))+Q[\varphi](\epsilon x) \frac{\partial F_{2}}{\partial \Pi}(x ; \Pi(\epsilon x)) \cdot \dot{\Pi}(\epsilon x)\right] \text {. }
$$

From (C32) and the expansion of $\arctan 1 / \epsilon$ we have

$$
\begin{aligned}
F_{2}\left(\frac{\delta}{\epsilon}, \pi(\delta)\right]=\frac{\epsilon^{4}}{\delta^{2} \Pi^{2}(\delta)} & {\left[\frac{1}{\delta^{2}(1+v \beta \Pi(\delta))^{2}}+\frac{1}{\delta^{2}(1-v \beta \Pi(\delta))^{2}}\right.} \\
& \left.-\frac{1}{v \beta \Pi(\delta) \delta}\left[\frac{1}{\delta(1-v \beta \Pi(\delta))}-\frac{1}{\delta(1+v \beta \Pi(\delta))}\right]\right]+O\left(\epsilon^{6}\right),
\end{aligned}
$$

where the first term of the right-hand side of (C38) vanishes, as $\epsilon \rightarrow 0$. Therefore

$$
\frac{\partial \phi_{2}}{\partial \epsilon}(0 ; \delta)=\lim _{\epsilon \rightarrow 0} \int_{0}^{\delta / \epsilon} d x x\left[\dot{Q}(\epsilon x) F_{2}(x ; \Pi(\epsilon x))+Q(\epsilon x) \frac{\partial F_{2}}{\partial \Pi}(x ; \Pi(\epsilon x)) \dot{\Pi}(\epsilon x)\right] \text {. }
$$

We apply to that integrand the mean value theorem, and after the same arguments as in cases (a) and (b), taking into account that the derivative with respect to $\epsilon$ is of order of $1 / x^{2}$, as $x \rightarrow \infty$, we obtain

$$
\frac{\partial \phi_{2}}{\partial \epsilon}(0 ; \delta)=\int_{0}^{\infty} x\left[\dot{Q}(0+) F_{2}(x ; 1)+Q(0+) \frac{\partial F_{2}}{\partial \pi}(x ; 1) \dot{\Pi}(0)\right] d x \text {. }
$$

Defining

$$
\begin{aligned}
& C_{1}=\int_{0}^{\infty} x F_{2}(x ; 1) d x, \\
& C_{2}=\int_{0}^{\infty} x \frac{\partial F_{2}}{\partial \Pi}(x ; 1) d x,
\end{aligned}
$$

we have

$$
\frac{\partial \phi_{2}}{\partial \epsilon}(0 ; \delta)=\dot{Q}(0+) C_{1}+Q(0+) \dot{\Pi}(0) C_{2}=2 \beta^{2} T_{2}[\varphi]
$$

The expressions $C_{1}$ and $C_{2}$ will be calculated later. Now, consider the second derivative of $\phi_{2}$. From (C38), we have

$$
\begin{aligned}
\frac{\partial^{2} \phi_{2}}{\partial \epsilon^{2}}= & \left.\frac{2 \delta}{\epsilon^{3}} Q[\varphi](\delta) F_{2}\left[\frac{\delta}{\epsilon} ; \Pi(\delta)\right]-\frac{\delta}{\epsilon^{2}} Q[\varphi](\delta) \frac{\partial F_{2}}{\partial x}\left(\frac{\delta}{\epsilon}, \Pi(\delta)\right]-\frac{\delta}{\epsilon^{2}}\right] \\
& -\frac{\delta}{\epsilon^{2}} \frac{\delta}{\epsilon}\left[\dot{Q}[\varphi](\delta) F_{2}\left[\frac{\delta}{\epsilon} ; \Pi(\delta)\right]+Q[\varphi](\delta) \frac{\partial F_{2}}{\partial \Pi}\left[\frac{\delta}{\epsilon} ; \Pi(\delta)\right] \dot{\Pi}(\delta)\right] \\
& +\int_{0}^{\delta / \epsilon} x^{2} d x\left[\ddot{Q}[\varphi](\epsilon x) F_{2}(x ; \Pi(\epsilon x))+2 \dot{Q}[\varphi](\epsilon x) \frac{\partial F_{2}}{\partial \Pi}(x ; \Pi(\epsilon x)) \dot{\Pi}(\epsilon x)\right. \\
& \left.+Q[\varphi](\epsilon x) \frac{\partial^{2} F_{2}}{\partial \Pi^{2}}(x ; \Pi(\epsilon x)) \dot{\Pi}^{2}(\epsilon x)+Q[\varphi](\epsilon x) \frac{\partial F_{2}}{\partial \Pi}(x ; \Pi(\epsilon x)) \ddot{\Pi}(\epsilon x)\right] .
\end{aligned}
$$


Now, $F_{2}(x, \Pi) \sim 1 / x^{4}, \partial F_{2} / \partial x \sim 1 / x^{5}$, and $\partial F_{2} / \partial \Pi \sim 1 / x^{4}$ as $x \rightarrow \infty$, then

$$
F_{2}\left(\frac{\delta}{\epsilon} ; \Pi\right) \sim \epsilon^{4}, \frac{\partial F_{2}}{\partial x}\left(\frac{\delta}{\epsilon} ; \Pi\right) \sim \epsilon^{5}, \text { and } \frac{\partial F_{2}}{\partial \Pi}\left(\frac{\delta}{\epsilon} ; \Pi\right) \sim \epsilon^{4} .
$$

Therefore, the two first terms of (C43) vanish as $\epsilon \rightarrow 0$. With respect to the integral we apply the mean value theorem and use the same arguments as in previous cases. The result we obtain is

$$
\begin{aligned}
\lim _{\epsilon \rightarrow 0} \frac{\partial^{2} \phi_{2}}{\partial \epsilon^{2}}=\int_{0}^{\infty} x^{2}\left[\ddot{Q}(0+) F_{2}(x ; 1)+2 \dot{Q}(0+) \frac{\partial F_{2}}{\partial \Pi}(x ; 1) \Pi(0)\right. \\
\\
\left.+Q(0+) \frac{\partial^{2} F_{2}}{\partial \Pi^{2}}(x ; 1) \dot{\Pi}^{2}(0)+Q(0+) \frac{\partial F_{2}}{\partial \Pi}(x ; 1) \ddot{\Pi}(0)\right] d x=2 \beta^{2} D_{2} .
\end{aligned}
$$

Now, we apply the Taylor formula up to the second derivative, to the function $\phi_{2}$,

$$
\phi_{2}(\epsilon ; \delta)=\phi_{2}(0 ; \delta)+\epsilon \frac{\partial \phi_{2}}{\partial \epsilon}(0 ; \delta)+\left.\frac{\epsilon^{2}}{2} \frac{\partial^{2} \phi_{2}}{\partial \epsilon^{2}}\right|_{\epsilon^{\prime}}, \epsilon^{\prime}<\epsilon
$$

and using (C29), (C30a) - (C30c), (C37), (C42), (C44), and (C45)

$$
K_{2}=\frac{1}{\epsilon} T_{2}[\varphi]+D_{2}+r_{5}(\epsilon) \text {. }
$$

Using the same notation as in the case of $K_{1}$ it turns out that

$$
\bar{K}_{2}=\frac{1}{\epsilon} \bar{T}_{2}[\varphi]+\bar{D}_{2}+\bar{r}_{5}(\epsilon) .
$$

Easily it can be seen that $\bar{T}_{2}=-T_{2}$ and $\bar{D}_{2}=D_{2}$, whereby

$$
K_{2}=\frac{1}{\epsilon} T_{2}+\frac{1}{2} \int_{-\infty}^{+\infty} d u I_{2}(u ; 0)+R_{2}(\epsilon) .
$$

It remains to calculate $(\mathrm{C} 41 \mathrm{a})$ and $(\mathrm{C} 41 \mathrm{~b})$. From $(\mathrm{C} 32)$,

$$
\begin{aligned}
& C_{1}=\int_{0}^{\infty} \frac{1}{x} {\left[\frac{1}{1+x^{2}(1+\beta)^{2}}+\frac{1}{1+x^{2}(1-\beta)^{2}}-\frac{1}{\beta x}[\arctan x(1+\beta)-\arctan x(1-\beta)]\right] d x } \\
& C_{2}=\int_{0}^{\infty} \frac{1}{x}\left[-\frac{2 x^{2}(1+\beta) \beta}{\left[1+x^{2}(1+\beta)^{2}\right]^{2}}+\frac{2 x^{2}(1-\beta) \beta}{\left[1+x^{2}(1-\beta)^{2}\right]^{2}}+\frac{3}{\beta x}[\arctan x(1+\beta)-\arctan x(1-\beta)]\right. \\
&\left.-\frac{3}{\beta x}\left[\frac{x \beta}{1+x^{2}(1+\beta)^{2}}+\frac{x \beta}{1+x^{2}(1-\beta)^{2}}\right]\right] d x .
\end{aligned}
$$

Using the integrals $S_{1}, S_{2}, S_{3}, S_{4}$, and also

$$
S_{5}(a)=\int d x \frac{1}{x^{2}} \arctan (x a)=-\frac{\arctan (x a)}{x}+a S_{2}(a)
$$

we obtain the following expressions:

$$
\begin{aligned}
& C_{1}=\left(-2+\frac{1}{\beta} \ln \frac{1+\beta}{1-\beta}\right), \\
& C_{2}=\left(2 \beta^{2} \gamma^{2}+6-\frac{3}{\beta} \ln \frac{1+\beta}{1-\beta}\right) .
\end{aligned}
$$

Substituting (C1g), (C11), and (C1m) and considering (C28) and (C42) we obtain 


$$
\begin{aligned}
T[\varphi]=T_{1}+T_{2}+T_{3} & \left.=\left(\vec{\varphi}_{p} \cdot \dot{\vec{\beta}}\right)\left(\frac{1}{4 \beta} \ln \frac{1+\beta}{1-\beta}+\frac{1}{4 \beta^{2}} C_{1}\right)+\left(\vec{\varphi}_{p} \cdot \vec{\beta}\right) \frac{\vec{\beta} \cdot \dot{\vec{\beta}}}{\beta^{2}} \mid-\frac{1}{4 \beta} \ln \frac{1+\beta}{1-\beta}+\frac{3 C_{1}}{4 \beta^{2}}+\frac{C_{2}}{2 \beta^{2}}\right) \\
& =d_{1}(\beta) \Omega_{1}[\varphi]+d_{2}(\beta) \Omega_{2}[\varphi]
\end{aligned}
$$

where we have used (C49a) and (C49b), and $d_{1}, d_{2}, \Omega_{1}$, and $\Omega_{2}$ are given by (5.40a) and (5.40b) and (5.41a) and (5.41b). This ends the proof of (5.38) and (5.39) and, consequently, (5.42).

\section{APPENDIX D}

The case of central potentials has great interest because it can be shown that, if the Lorentz-Dirac damping term behaves like a vector with respect to rotations and the correlation of the stochastic force like a dyadic product, the solution of the FokkerPlanck equation depends only on constants which are invariant by rotations. Then, the process of reduction of the coefficients of that equation can be made to these constants alone. This is a great advantage because it allows the simplification of the problem of the symmetric character of the diffusion matrix $G^{\mu \nu}$, so that, for any spectral densi- ty, it can be shown that $G^{\mu \nu}=G^{v \mu}$ if the potential is central. Therefore, in the particular case of a Rayleigh-Jeans spectrum, the proof that the equilibrium density is the Maxwell-Boltzmann one simplifies notably. (In the following we do not use the notation of Sec. II C.)

The damping term in $(2.2 \mathrm{~b})$ is

$$
\overrightarrow{\mathrm{F}}^{d}=\frac{2 e^{2}}{3 c^{3}}\left(\dot{\vec{\alpha}}-\frac{1}{c^{2}} \alpha_{v} \alpha^{v} \overrightarrow{\mathrm{v}}\right),
$$

whose vector character is straightforward from (5.2). On the other hand, the correlation of the stochastic force is given by

$$
\begin{aligned}
& \left\langle F_{r}^{\mathrm{st}}(\overrightarrow{\mathrm{r}}, \overrightarrow{\mathrm{p}}, t) F_{l}^{\mathrm{st}}\left(\overrightarrow{\mathrm{r}}^{\prime}, \overrightarrow{\mathrm{p}}^{\prime}, t^{\prime}\right)\right\rangle=\frac{e^{2}}{c^{3}} \int_{0}^{\infty} d \omega \frac{\omega^{2} \mathscr{Y}^{2}(\omega)}{2} \int d \Omega \cos \left[\frac{\omega}{c} \hat{r}^{0} \cdot\left(\overrightarrow{\mathrm{r}}-\overrightarrow{\mathrm{r}}^{\prime}\right)-\omega\left(t-t^{\prime}\right)\right] \\
& \times\left[\left(\delta_{r l}-r_{r}^{0} r_{l}^{0}\right)+\sum_{i j s} \epsilon_{l i j} \epsilon_{r j s} \beta_{i}^{\prime} r_{s}^{0}+\sum_{i j s} \epsilon_{r i j} \epsilon_{l j s} \beta_{i} r_{s}^{0}\right. \\
& \left.+\sum_{i j \alpha \sigma} \epsilon_{r i j} \epsilon_{l \alpha \sigma} \beta_{i} \beta_{\alpha}^{\prime}\left(\delta_{j \sigma}-r_{j}^{0} r_{\sigma}^{0}\right)\right]
\end{aligned}
$$

Here, the tensor character of the expression $\delta_{i j}-r_{i}^{0} r_{j}^{0}$ and the quantity $\epsilon_{i j k}$ guarantee the tensor character of the correlation.

Consequently, the problem can be reduced to the constants which are invariant by rotations alone. Let us see which are these constants. The relativistic Hamilton-Jacobi equation for a particle in a central field can be written

$$
V(r)+\left\{m^{2} c^{4}+c^{2}\left[\left(\frac{\partial W}{\partial r}\right]^{2}+\frac{1}{r^{2}}\left[\frac{\partial W}{\partial \theta}\right]^{2}+\frac{1}{r^{2} \sin ^{2} \theta}\left[\frac{\partial W}{\partial \varphi}\right]^{2}\right]\right\}^{1 / 2}=\mathscr{E},
$$

which can be separated. The action variables $\bar{J}_{1}$ are the same as in the nonrelativistic case except that $\bar{J}_{1}$ is given now by

$$
\bar{J}_{1}=\frac{1}{2 \pi} \oint p_{r} d r=\frac{1}{\pi} \int_{r_{m}}^{r_{M}}\left[\frac{(\mathscr{E}-V(r))^{2}}{c^{2}}-m^{2} c^{2}-\frac{L^{2}}{r^{2}}\right]^{1 / 2} d r
$$

Through a linear canonical transformation, in the same way as in the nonrelativistic case, we define the new variables as 


$$
\begin{aligned}
& J_{1}=\bar{J}_{1}, \\
& J_{2}=\bar{J}_{2}+\bar{J}_{3}=L, \\
& J_{3}=\bar{J}_{3}=L_{z},
\end{aligned}
$$

so that the energy depends only on the first two, i.e.,

$$
\mathscr{C}=\mathscr{C}\left(J_{1}, J_{2}\right)
$$

and, therefore, $\omega_{3}=0$

Only the first two constants of action are invariant by rotations. Therefore, the reduction process must be made by defining the operator

$$
\begin{aligned}
T[\varphi] & =\int d \vec{\xi} \prod_{i} \delta\left(h_{i}-h_{i}(\vec{\xi})\right) \varphi(\vec{\xi})=\int d \vec{\xi} \delta\left(J_{1}-J_{1}(\vec{\xi})\right) \delta\left(J_{2}-J_{2}(\vec{\xi})\right) \varphi(\vec{\xi}) \\
& =\int_{-J_{2}}^{+J_{2}} d J_{3} \int_{0}^{2 \pi} d w_{1} \int_{0}^{2 \pi} d w_{2} \int_{0}^{2 \pi} d w_{3} \varphi\left(J_{1}, J_{2}, J_{3}, w_{1}, w_{2}, w_{3}\right)
\end{aligned}
$$

in such a way that we add the contributions of the motions described by each orbit in the reverse sense.

This is related to the way in which the coefficients are transformed by time reversal. We see that $J_{1}$ and $J_{2}$, aside from being by rotations, are also time-reversal invariant. We shall use this property in order to prove the symmetry of the diffusion matrix. To do that, we start by generalizing a result already proved in the case that the stochastic force does not depend on the phase variables. ${ }^{19}$ Let

$$
B_{i j}(\zeta, u)=\left\langle K_{i}(\zeta, 0) K_{j}\left(\xi^{-u},-u\right)\right\rangle
$$

be the correlation of the stochastic force which appears in the expression of $G^{\mu \nu}$. Let $\widetilde{\xi}$ represent the same spatial point as $\zeta$, but with the velocity in the reverse sense. In our case $\xi=(\vec{r}, \vec{p}) \Longrightarrow \widetilde{\xi}=(\vec{r},-\vec{p})$. We shall write $\widetilde{\zeta}_{i}=\epsilon_{i} \xi_{i}$ with $\epsilon_{i}= \pm 1$ according to the value of the index $i$. We shall show the following general proposition.

If $B_{i j}$ fulfills the relation

$$
B_{r l}(\xi, u)=\epsilon_{r} \epsilon_{l} B_{l r}\left(\widetilde{\zeta}^{u}, u\right)
$$

then the diffusion coefficients fulfill

$$
O_{T} G^{\mu v}=\epsilon_{\mu} \epsilon_{v} G^{v \mu} .
$$

Let us assume also that $\widetilde{h}_{\mu}=\epsilon_{\mu} h_{\mu}$ with $\epsilon_{\mu}= \pm 1$. If this is not true, as $O_{T}^{2}=I$ and it has eigenvalues \pm 1 , it is possible to find linear combinations that fulfill it. In order to show (D10) we use (3.3b) and (D7) to obtain

$$
O_{T} G^{\mu \nu}=G^{\mu \nu}\left(\epsilon_{i} h_{i}\right)=\alpha^{2} \int d \vec{\xi} \prod_{i} \delta\left(\epsilon_{i} h_{i}-h_{i}(\vec{\xi})\right) \int_{0}^{\infty} d u \sum_{r, l} \frac{\partial h_{\mu}}{\partial \xi_{r}} \frac{\partial h_{v}}{\partial \xi_{l}^{-u}} B_{r l}(\xi, u)
$$

We perform now the change of variables $\zeta_{i} \rightarrow \widetilde{\zeta}_{i}=\epsilon_{i} \zeta_{i}$. The following relations hold:

$$
\begin{aligned}
& \widetilde{\zeta}_{i}^{-u}=\epsilon_{i} \zeta_{i}^{u}=\widetilde{\zeta}_{i}^{u}, \\
& \zeta_{i}^{-u}=\left(\epsilon_{i} \widetilde{\zeta}_{i}\right)^{-u}=\epsilon_{i} \widetilde{\zeta}_{i}{ }^{u}, \\
& h_{\mu}\left(\epsilon_{r} \widetilde{\xi}_{r}\right)=\epsilon_{\mu} h_{\mu}\left(\widetilde{\xi}_{r}\right)=h_{\mu}\left(\xi_{r}\right), \\
& \frac{\partial h_{\mu}}{\partial \xi_{r}}=\epsilon_{\mu} \frac{\partial h_{\mu}\left(\widetilde{\zeta}_{r}\right)}{\partial \widetilde{\zeta}_{r}} \frac{\partial \widetilde{\xi}_{r}}{\partial \xi_{r}}=\epsilon_{\mu} \epsilon_{r} \frac{\partial h_{\mu}\left(\widetilde{\xi}_{r}\right)}{\partial \widetilde{\xi}_{r}}, \\
& \frac{\partial h_{v}}{\partial \xi_{l}^{-u}}=\frac{\partial h_{v}\left(\xi_{i}^{-u}\right)}{\partial \xi_{l}^{-u}}=\frac{\partial h_{v}\left(\epsilon_{i} \widetilde{\zeta}^{u}\right)}{\partial\left(\epsilon_{l} \widetilde{\zeta}_{l}^{u}\right)}=\epsilon_{v} \epsilon_{l} \frac{\partial h_{v}\left(\widetilde{\zeta}_{i}{ }^{u}\right)}{\partial \widetilde{\xi}_{l}{ }^{u}} .
\end{aligned}
$$


Then (D11) becomes

$$
O_{T} G^{\mu v}=\alpha^{2} \int d \widetilde{\xi} \prod_{i} \delta\left(h_{i}-h_{i}(\widetilde{\xi})\right) \int_{0}^{\infty} d u \sum_{r, l} \frac{\partial h_{\mu}}{\partial \widetilde{\xi}_{r}} \frac{\partial h_{v}}{\partial \widetilde{\xi}_{l}^{u}} \epsilon_{\mu} \epsilon_{v} \epsilon_{r} \epsilon_{l} B_{r l}\left(\epsilon_{i} \widetilde{\xi}_{i}, u\right) .
$$

The new change $\zeta_{i}^{\prime}=\widetilde{\zeta}_{i}{ }^{u} \Longrightarrow \widetilde{\zeta}_{i}=\zeta^{\prime}{ }_{i}^{-u}$ gives

$$
O_{T} G^{\mu v}=\alpha^{2} \int d \zeta^{\prime} \prod_{i} \delta\left(h_{i}-h_{i}\left(\xi^{\prime}\right)\right) \int_{0}^{\infty} d u \sum_{r, l} \frac{\partial h_{v}}{\partial \zeta_{l}^{\prime}} \frac{\partial h_{\mu}}{\partial \xi_{r}^{\prime-u}} \epsilon_{\mu} \epsilon_{v} \epsilon_{r} \epsilon_{l} B_{r l}\left(\epsilon_{i} \zeta_{i}^{\prime-u}, u\right) .
$$

Now $\epsilon_{i} \xi_{i}^{\prime-u}=\widetilde{\xi}_{i}^{\prime}$ and, by condition (D9),

$$
\epsilon_{r} \epsilon_{l} B_{r l}\left(\widetilde{\zeta}_{i}^{\prime}, u\right)=B_{l r}\left(\zeta_{i}^{\prime}, u\right)
$$

whence

$$
O_{T} G^{\mu v}=\epsilon_{\mu} \epsilon_{v} \alpha^{2} \int d \zeta^{\prime} \prod_{i} \delta\left(h_{i}-h_{i}\left(\zeta^{\prime}\right)\right) \int_{0}^{\infty} d u \sum_{r, l} \frac{\partial h_{v}}{\partial \zeta_{l}^{\prime}} \frac{\partial h_{\mu}}{\partial \xi_{r}^{\prime-u}} B_{l r}\left(\zeta_{i}^{\prime}, u\right)=\epsilon_{\mu} \epsilon_{v} G^{v \mu}
$$

which completes the proof.

Now, we apply this result to our particular case. We start by considering the implications of Eq. (D9) for this case. As $\zeta \equiv(\vec{r}, \vec{p})$ and $\widetilde{\xi} \equiv(\vec{r},-\vec{p})$, then $\epsilon_{1}=\epsilon_{2}=\epsilon_{3}=1$ and $\epsilon_{4}=\epsilon_{5}=\epsilon_{6}=-1$. Moreover, $B_{r l}$ is not zero only for $r, l>3$. Putting this into (D8), Eq. (D9) is written

$$
\begin{aligned}
\left\langle F_{r}^{\mathrm{st}}(\overrightarrow{\mathrm{r}}, \overrightarrow{\mathrm{p}}, 0) F_{l}^{\mathrm{st}}\left(\overrightarrow{\mathrm{r}}^{-u}, \overrightarrow{\mathrm{p}}^{-u},-u\right)\right\rangle \\
\quad=\left\langle F_{l}^{\mathrm{st}}\left(\overrightarrow{\mathrm{r}}^{-u},-\overrightarrow{\mathrm{p}}^{-u}, 0\right) F_{r}^{\mathrm{st}}(\overrightarrow{\mathrm{r}},-\overrightarrow{\mathrm{p}},-u)\right\rangle
\end{aligned}
$$

because $\widetilde{\zeta}^{u}=\tilde{\zeta}^{\tilde{-}}$.

In order to understand the physical meaning of (D13) let us consider a path and assume that the particle follows it in a given sense which we label with a plus. Then, the point $\vec{r}, \vec{p}$ will be written $\overrightarrow{\mathrm{r}}_{1}^{+}$and the point $\overrightarrow{\mathrm{r}}^{-u}, \overrightarrow{\mathrm{p}}^{-u}$ as $\overrightarrow{\mathrm{r}}_{2}^{+}$. On the lefthand side of (D13), we calculate the component $r$ of the force in the point $\overrightarrow{\mathrm{r}}_{1}^{+}$at time $t=0$, and the component $l$ at the point $\overrightarrow{\mathrm{r}}_{2}^{+}$at time $t=-u$. This is the same as putting the component $r$ in the point $\vec{r}_{1}^{+}$and the component $l$ in the point $\vec{r}_{2}^{+}$, the former at a time $u$ after. [Note in (D2) that the explicit dependence with respect to the times in the correlations is through their difference]. In the motion with the stated sense, $\vec{r}_{2}^{+}$is reached before $\overrightarrow{\mathbf{r}}_{1}^{+}$, so that $F_{l}$ is calculated before at the point where the particle is earlier.

On the right-hand side of (D13), $F_{r}$ is calculated at $r_{1}$, but the sense of the motion is the contrary, $\overrightarrow{\mathrm{r}}_{1}^{-}$, and at time $-u$. Also $F_{l}$ is calculated at $\overrightarrow{\mathrm{r}}_{2}$, also with the reverse sense of the motion, $\vec{r}_{2}^{-}$, at time 0 .

We see, therefore, that $F_{r}$ and $F_{l}$ are calculated both at the same point of the path, in such a way that the first one calculated is the first one to "see" the particle. In summary, it gives the same result the calculation of the correlation between two points of the same path with independence of the sense of the motion provided that we put the value of the field at the time in which the particle is at that point. Also, it is indifferent to calculate before either point provided that the sense of the motion is such that we calculate before the field of the point where the particle is first.

Let us see that relation (D13) is fulfilled in our case. To do that we use (D2) which leads to

$$
\begin{aligned}
& \left\langle F_{r}^{\mathrm{st}}(\overrightarrow{\mathrm{r}}, \overrightarrow{\mathrm{p}}, 0) F_{l}^{\mathrm{st}}\left(\overrightarrow{\mathrm{r}}^{-u}, \overrightarrow{\mathrm{p}}^{-u},-u\right)\right\rangle \\
& =\frac{e^{2}}{c^{3}} \int_{0}^{\infty} d \omega \frac{\omega^{2} \mathscr{Y}^{2}(\omega)}{2} \int d \Omega \cos \left[\frac{\omega}{c} \hat{r}^{0} \cdot\left(\overrightarrow{\mathrm{r}}-\overrightarrow{\mathrm{r}}^{-u}\right)-\omega u\right] \\
& \quad+\sum_{i j \alpha \sigma} \epsilon_{r i j} \epsilon_{l \alpha \sigma} \beta_{i} \beta_{\alpha}^{-u}\left(\delta_{j \sigma}-r_{r}^{0} r_{\sigma}^{0} r_{\sigma}^{0}\right)+\sum_{i j s}\left(\epsilon_{l i j} \epsilon_{r j s} \beta_{i}^{-u} r_{s}^{0}+\epsilon_{r i j} \epsilon_{l j s} \beta_{i} r_{s}^{0}\right)
\end{aligned}
$$


and

$$
\begin{aligned}
\left\langle F_{l}^{\mathrm{st}}\left(\overrightarrow{\mathrm{r}}^{-u},-\overrightarrow{\mathrm{p}}^{-u}, 0\right) F_{r}^{\mathrm{st}}(\overrightarrow{\mathrm{r}},-\overrightarrow{\mathrm{p}},-u)\right\rangle & =\frac{e^{2}}{c^{3}} \int_{0}^{\infty} d \omega \frac{\omega^{2} \mathscr{Y}^{2}(\omega)}{2} \int d \Omega \cos \left[\frac{\omega}{c} \hat{r}^{0} \cdot\left(\overrightarrow{\mathrm{r}}^{-u}-\overrightarrow{\mathrm{r}}\right)-\omega u\right] \\
\times & \left\{\left(\delta_{l r}-r_{l}^{0} r_{r}^{0}\right)+\sum_{i j s}\left[\epsilon_{r i j} \epsilon_{l j s}\left(-\beta_{i}\right) r_{s}^{0}+\epsilon_{l i j} \epsilon_{r j s}\left(-\beta_{i}^{-u}\right) r_{s}^{0}\right]\right. \\
& \left.+\sum_{i j \alpha \sigma} \epsilon_{l i j} \epsilon_{r \alpha \sigma}\left(-\beta_{i}^{-u}\right)\left(-\beta_{\alpha}\right)\left(\delta_{j \sigma}-r_{j}^{0} r_{\sigma}^{0}\right)\right\} .
\end{aligned}
$$

Performing the change $\hat{r}^{\prime 0}=-\hat{r}^{0}$, we have

$$
\begin{aligned}
& \left\langle F_{l}^{\mathrm{st}}\left(\overrightarrow{\mathrm{r}}^{-u},-\overrightarrow{\mathrm{p}}^{-u}, 0\right) F_{r}^{\mathrm{st}}(\overrightarrow{\mathrm{r}},-\overrightarrow{\mathrm{p}},-u)\right\rangle \\
& =\frac{e^{2}}{c^{3}} \int_{0}^{\infty} d \omega \frac{\omega^{2} \mathscr{Y}^{2}(\omega)}{2} \int d \Omega \cos \left[-\frac{\omega}{c} \hat{r}^{0} \cdot\left(\overrightarrow{\mathrm{r}}^{-u}-\overrightarrow{\mathrm{r}}\right)-\omega u\right] \\
& \times\left[\left(\delta_{r l}-r_{r}^{0} r_{l}^{0}\right)+\sum_{i j s}\left(\epsilon_{r i j} \epsilon_{l j s} \beta_{i} r_{s}^{0}+\epsilon_{l i j} \epsilon_{r j s} \beta_{i}^{-u} r_{s}^{0}\right)+\sum_{i j \alpha \sigma} \epsilon_{l \alpha \sigma} \epsilon_{r i j} \beta_{\alpha}^{-u} \beta_{i}\left(\delta_{j \sigma}-r_{j}^{0} r_{\sigma}^{0}\right)\right],
\end{aligned}
$$

(D15)

where in the last term we have changed the indices $i$ by $\alpha$ and $j$ by $\sigma$.

We see that (D15) and (D14) are equal and, therefore, relation (D13) is fulfilled. As a consequence we may write

$$
G^{\mu \nu}\left(\epsilon_{\rho} h_{\rho}\right)=\epsilon_{\mu} \epsilon_{v} G^{v \mu}\left(h_{\rho}\right) .
$$

Now, as the only constants present are time-reversal invariant, it results $\epsilon_{\mu}=1, \mu=1,2$ whence

$$
G^{\mu \nu}\left(h_{\rho}\right)=G^{v \mu}\left(h_{\rho}\right) \text {, }
$$

which completes the proof.

${ }^{1}$ A. Pais, Rev. Mod. Phys. 51, 863 (1979). This paper reviews the early contributions of Einstein and others on the subject.

2J. H. Van Vleck and D. L. Huber, Rev. Mod. Phys. 49, 939 (1977), reviews the situation around 1924.

${ }^{3}$ T. H. Boyer, Phys. Rev. A 20, 1246 (1979).

${ }^{4}$ T. H. Boyer, Phys. Rev. D $\underline{19}, 3635$ (1979).

5Uri Ben-Ya'acov, Phys. Rev. D 23, 1441 (1981); A. Peres, ibid. 23, 1458 (1981); C. H. Braden, R. F. Fox, and H. A. Gersch, ibid. 23, 1455 (1979).

${ }^{6}$ T. H. Boyer, Phys. Rev. 182, 1374 (1969).

${ }^{7}$ T. H. Boyer, in Proceedings of the Boulder Conference on Foundations of Radiation Theory and Quantum Electrodynamics, edited by A. O. Barut (Plenum, New York, 1979).

${ }^{8} \mathrm{~F}$. Rohrlich, Classical Charged Particles (AddisonWesley, Reading, Mass., 1965).

${ }^{9}$ T. H. Boyer, Phys. Rev. D $\underline{11}, 809$ (1975).

${ }^{10}$ E. Santos, Nuovo Cimento 22B, 201 (1974).

${ }^{11}$ H. Goldstein, Classical Mechanics, 2nd ed. (AddisonWesley, Reading, Mass., 1980).
${ }^{12}$ F. Gantmacher, Lectures in Analytical Mechanics (MIR Publishers, Moscow, 1975).

${ }^{13}$ L. Arnold, Stochastic Differential Equations: Theory and Applications (Wiley, New York, 1974).

${ }^{14} \mathrm{P}$. Claverie, in Proceedings of the Einstein Centennial Symposium on Fundamental Physics, edited by S. Moore et al. (Uniandes, Bogotá, 1981).

${ }^{15}$ M. Lax, Rev. Mod. Phys. $\underline{38}, 541$ (1966).

16N. G. Van Kampen, Phys. Rep. 24C, 171 (1976).

${ }^{17}$ R. Z. Khas'minskii, Theory Probab. Its Appl. USSR $\underline{11}, 390$ (1966). For a qualitative discussion see Ref. 15 , Sec. 5.

${ }^{18}$ H. Haken, Rev. Mod. Phys. 47, 67 (1975).

${ }^{19}$ L. Pesquera and P. Claverie (unpublished).

${ }^{20}$ R. Z. Khas'minskii, Theory Probab. Its Appl. USSR 5, 179 (1960).

21J. D. Jackson, Classical Electrodynamics (Wiley, New York, 1975).

${ }^{22}$ T. M. Apóstol, Mathematical Analysis (AddisonWesley, Reading, Mass., 1957). 